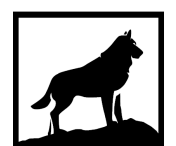

Michigan

Technological

1 8 8 5 University
Michigan Technological University

Digital Commons @ Michigan Tech

\title{
AN INJECTABLE THERMOSENSITIVE BIODEGRADABLE HYDROGEL EMBEDDED WITH SNAP CONTAINING PLLA MICROPARTICLES FOR SUSTAINED NITRIC OXIDE (NO) DELIVERY FOR WOUND HEALING
}

Nikhil Mittal

Michigan Technological University, nmittal@mtu.edu

Copyright 2018 Nikhil Mittal

\section{Recommended Citation}

Mittal, Nikhil, "AN INJECTABLE THERMOSENSITIVE BIODEGRADABLE HYDROGEL EMBEDDED WITH SNAP CONTAINING PLLA MICROPARTICLES FOR SUSTAINED NITRIC OXIDE (NO) DELIVERY FOR WOUND HEALING", Open Access Master's Thesis, Michigan Technological University, 2018.

https://doi.org/10.37099/mtu.dc.etdr/606

Follow this and additional works at: https://digitalcommons.mtu.edu/etdr

Part of the Biology and Biomimetic Materials Commons, Biomaterials Commons, and the Polymer and Organic Materials Commons 
AN INJECTABLE THERMOSENSITIVE BIODEGRADABLE HYDROGEL EMBEDDED WITH SNAP CONTAINING PLLA MICROPARTICLES FOR SUSTAINED NITRIC OXIDE (NO) DELIVERY FOR WOUND HEALING

By

Nikhil Mittal

\begin{abstract}
A THESIS
Submitted in partial fulfillment of the requirements for the degree of MASTER OF SCIENCE

In Biomedical Engineering
\end{abstract}

MICHIGAN TECHNOLOGICAL UNIVERSITY

2018

(C) 2018 Nikhil Mittal 
This thesis has been approved in partial fulfillment of the requirements for the Degree of MASTER OF SCIENCE in Biomedical Engineering.

Department of Biomedical Engineering

Thesis Advisor: $\quad$ Dr. Megan C. Frost

Committee Member: Dr. Smitha Rao

Committee Member: $\quad$ Dr. Lanrong Bi

Department Chair: Dr. Sean J. Kirkpatrick 
Table of Contents

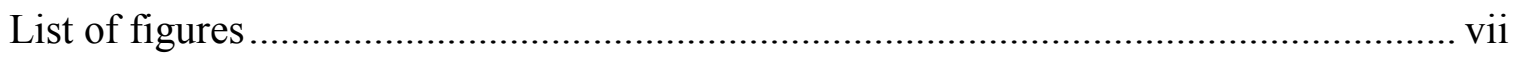

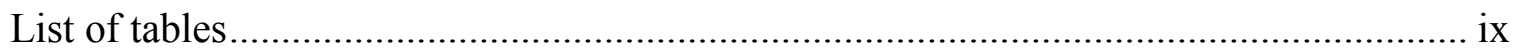

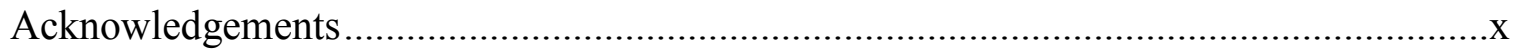

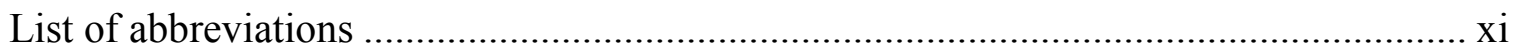

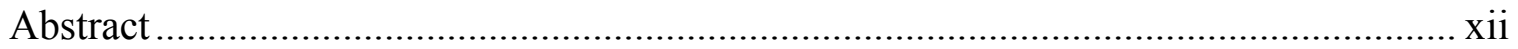

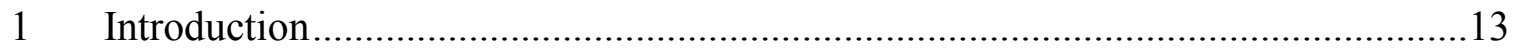

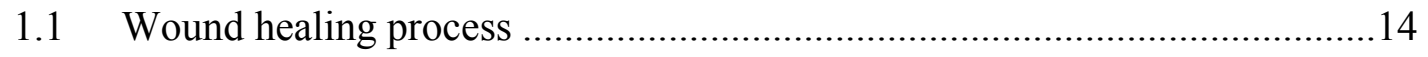

1.1.1 Wound healing complications.................................................. 18

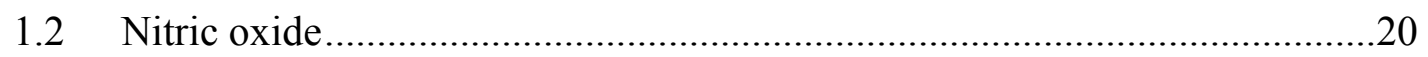

1.2.1 Role of nitric oxide in wound healing..........................................21

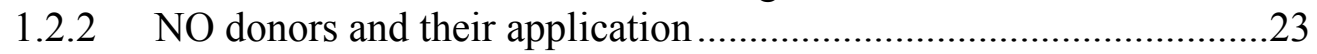

1.2.2.1 S-Nitrosothiols .......................................................24

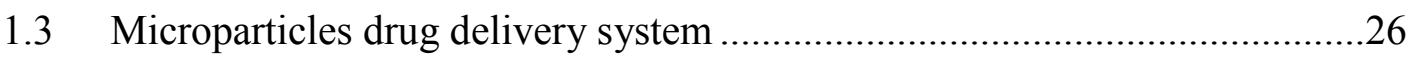

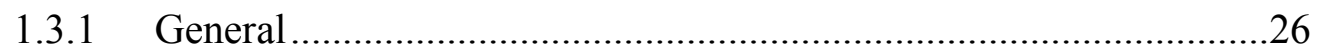

1.3.2 Different preparation methods of polymeric microparticles............27

1.3.2.1 Coacervation (precipitation by non-solvent addition) ....28

1.3.2.2 Emulsion salting out ...............................................28

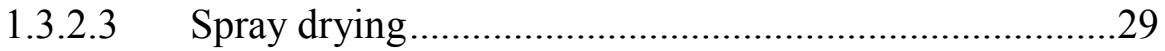

1.3.2.4 Solvent partitioning precipitation ...............................29

1.3.2.5 Supercritical fluid extraction method............................29

1.3.2.6 Solvent evaporation method ..........................................30

1.3.2.6.1 Single emulsion......................................30

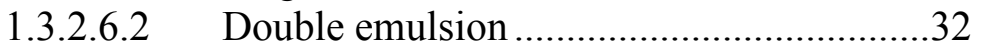

1.4 Microparticles preparation by solvent evaporation method ...........................33

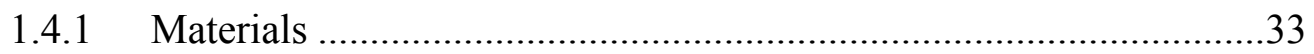

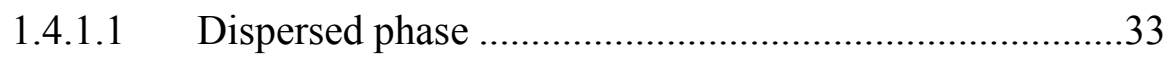

1.4.1.1.1 Polymer ....................................................33

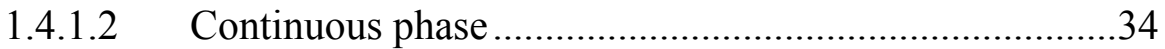

1.4.1.2.1 Surfactant ...............................................34

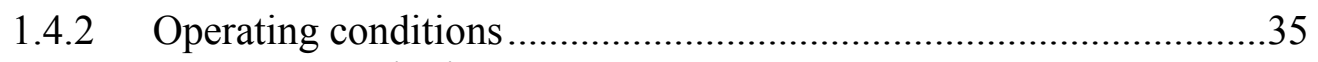

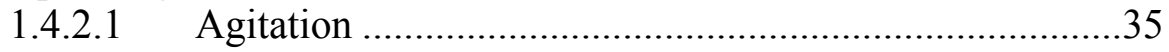

1.4.2.2 Temperature and pressure ..........................................35 


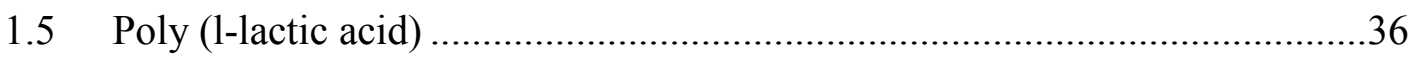

1.5.1 Drug release from PLA devices ......................................................37

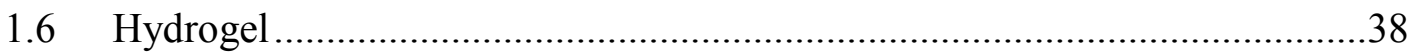

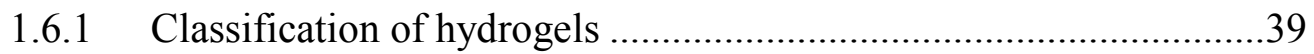

1.6.2 Hydrogels in wound therapy .......................................................... 41

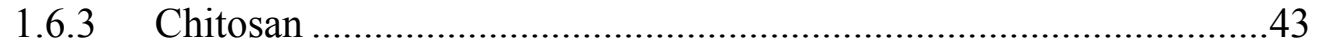

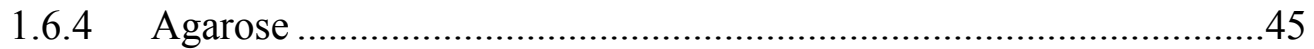

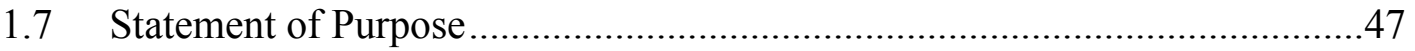

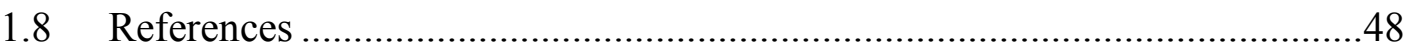

2 Particle synthesis and characterization ............................................................59

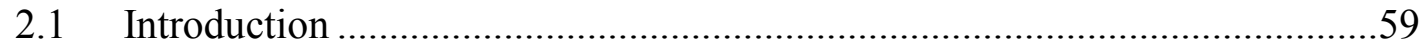

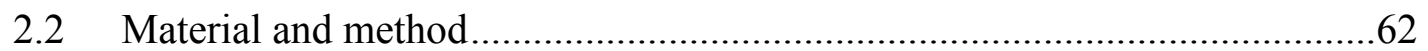

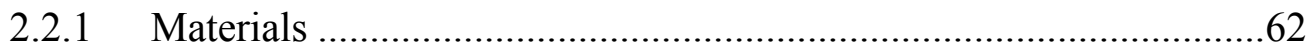

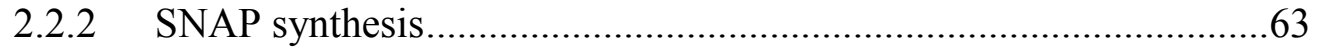

2.2.3 S-nitroso-N-acetylpenicillamine (SNAP) loaded poly(1-lactic)-

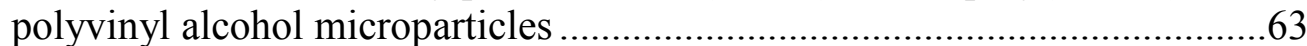

2.2.3.1 Preparation of SNAP loaded microparticles ....................63

2.2.4 Yield of the prepared microparticles...............................................65

2.2.5 Characterization of the SNAP loaded microparticles ........................65

2.2.6 UV-Vis analysis of SNAP loaded microparticles ...............................65

2.2.7 In vitro nitric oxide release study of SNAP loaded microparticles

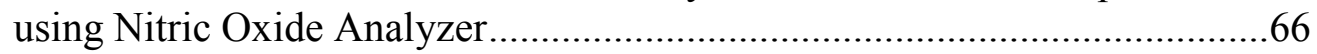

2.2.7.1 Controlled NO release analysis........................................66

2.2.7.2 Total NO release analysis .............................................66

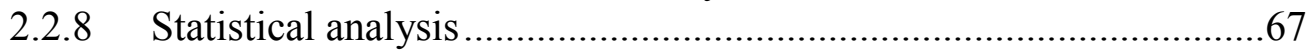

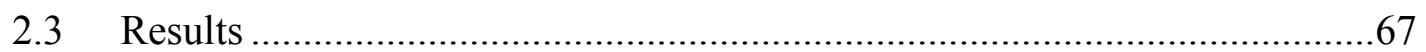

2.3.1 SNAP loaded PLLA microparticles.................................................67

2.3.2 Yield of the microparticles..............................................................67

2.3.3 Characterization of microparticles ....................................................68

2.3.3.1 Size and morphology of SNAP loaded microparticles ...68

2.3.3.2 SEM images ....................................................................

2.3.4 Encapsulation efficiency of the SNAP loaded microparticles ...........73

2.3.4.1 UV- Vis analysis of SNAP loaded microparticles ...........73

2.3.5 In vitro NO release profile study of SNAP loaded microparticles ...76 2.3.5.1 Controlled NO release study via Light ............................76

2.3.5.2 Effect of particle size on the light initiated controlled NO

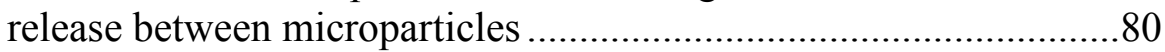

2.3.6 Total NO release study via light, Copper(II) ions and ascorbate.......81 
2.3.6.1 Effect of particle size on total NO release between

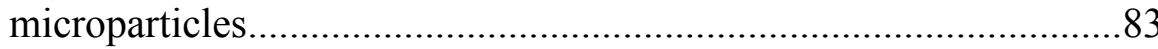

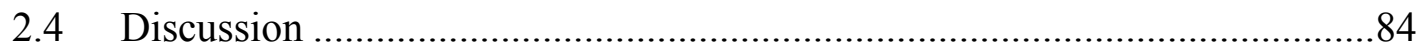

2.4.1 Size and characteristic of microparticle .............................................8

2.4.2 Encapsulation efficiency based on total NO release............................85

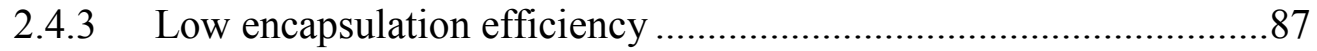

2.4.4 NO release profile and burst release ..................................................8

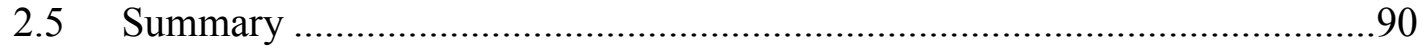

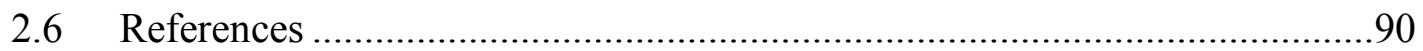

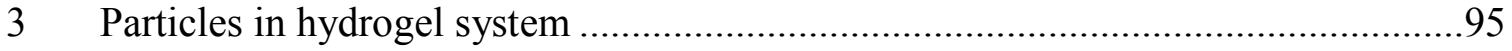

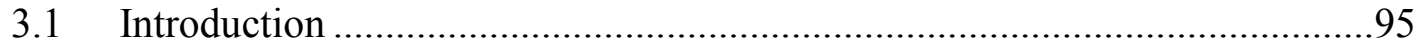

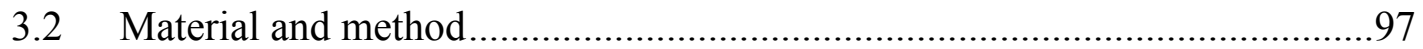

3.2.1 Preparation of SNAP loaded microparticles and chitosan-agarose

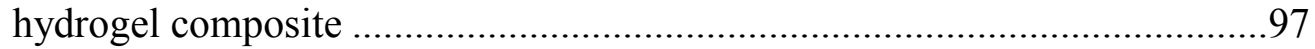

3.2.2 Preparation of ascorbic acid chitosan-agarose composite hydrogel .99

3.2.3 In vitro nitric oxide release study of Hydrogel-microparticles using

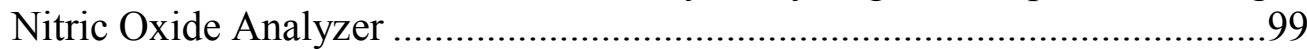

3.2.3.1 Total NO release using light .......................................100

3.2.3.2 Total NO release using ascorbic acid hydrogel system 100

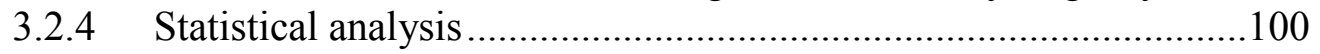

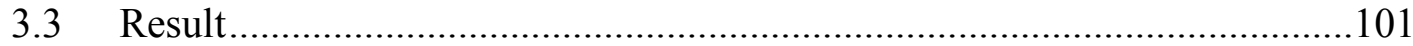

3.3.1 Microparticle Hydrogel Composite ..................................................101

3.3.2 Morphology of hydrogel-microparticle composite ...........................102

3.3.3 In vitro NO release profile study of microparticles hydrogel composite

3.3.3.1 Total NO release study via Light ...................................103

3.3.3.2 Effect of particle size on total NO release due to light in microparticle hydrogel composite .................................................106

3.3.4 Total NO release study via ascorbic acid hydrogel ........................107 3.3.4.1 Effect of particle size on total NO release due to ascorbic acid hydrogel in microparticle hydrogel composite ........................110

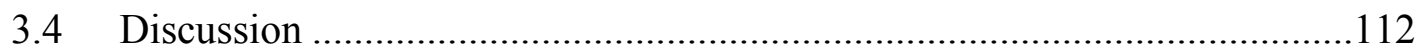

3.4.1 Hydrogel-microparticle composite …………...............................112

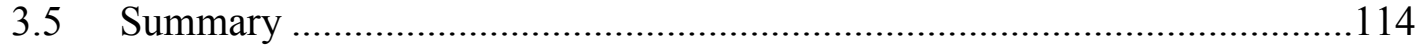

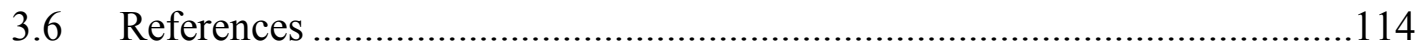




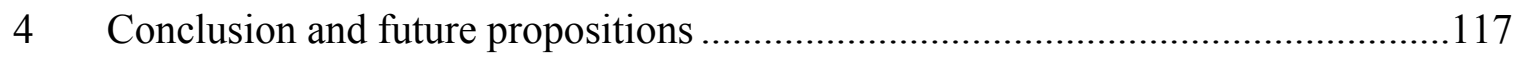

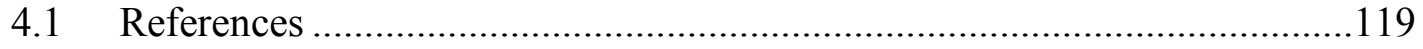

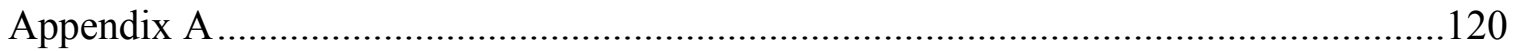




\section{List of figures}

Figure 1.1. Wound healing process (Hamdan. S. et al. 2017) (21)...................................17

Figure 1.2. Generation of NO during different phases of wound healing (Witte et al.

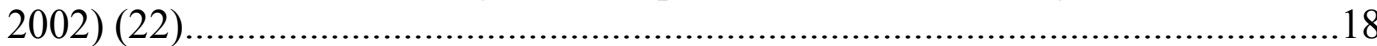

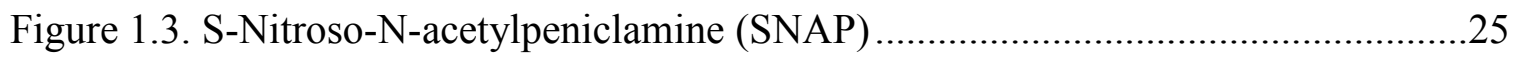

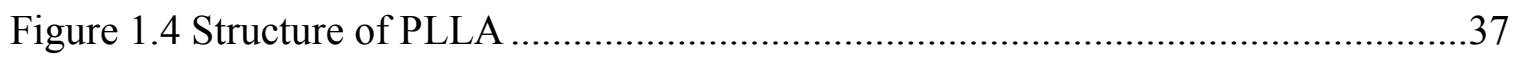

Figure 1.5 Microparticle encapsulation in hydrogel ....................................................42

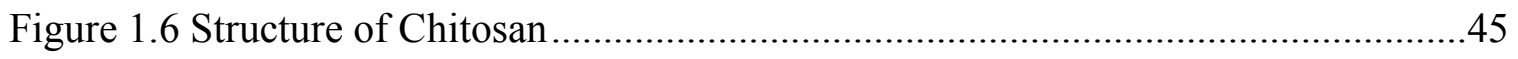

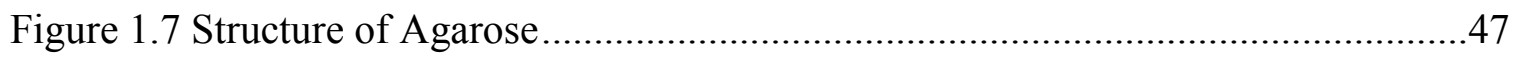

Figure 2.1 Preparation of the microparticles using double emulsion solvent evaporation method. Produced microparticles were collected, air dried and stored in dark at $20{ }^{\circ} \mathrm{C}$.

Figure 3.1 SEM images of microparticles made at 500 RPM (a),(c),(e),(g) shows the blank microparticle and SNAP loaded microparticle I,II,III respectively at magnification $1.00 \mathrm{~mm}$. (b),(d),(f),(h) surface topography of blank microparticle and SNAP loaded microparticle I,II,III respectively.

Figure 3.2 SEM images of microparticles made at 2500 RPM (i), (k),(m),(o) shows the blank microparticle and SNAP loaded microparticle I,II,III respectively at magnification $1.00 \mathrm{~mm} .(\mathrm{j}),(\mathrm{l}),(\mathrm{n}),(\mathrm{p})$ surface topography of blank microparticle and SNAP loaded microparticle I,II,III respectively.

Figure 3.3 Standard curve of SNAP in DCM:Methanol (3:2) …………………….........74

Figure 3.4 UV-vis analysis of SNAP loaded microparticles(500) $(\mathrm{n}=3) \ldots \ldots \ldots \ldots \ldots \ldots \ldots \ldots \ldots . . .75$

Figure 3.5 UV-vis analysis of SNAP loaded microparticles(500) $(\mathrm{n}=3) \ldots \ldots \ldots \ldots \ldots \ldots \ldots \ldots \ldots \ldots . . .75$

Figure 3.6 (A) Representative curve shows the controlled NO release from microparticle $(500)$. In the figures, trace (a) shows zero NO release in absence of light. (b) to (g) shows increasing and then decreasing NO release with varying intensit intensity of light at changing voltage.

Figure 3.7 Comparison of controlled light initiated NO release from microparticles(500) and microparticles(2500) 
Figure 3.8 Total NO release from the (a)microparticle $(500)$ (b) microparticles(2500) using light $(4 \mathrm{~V}), 50 \mu \mathrm{M} \mathrm{CuCl} 2$ and $100 \mathrm{mM}$ ascorbic acid.....

Figure 3.9 comparison of $\mathrm{NO}$ release rate from the microparticle(500) and microparticles $(2500)$

Figure 3.10 Comparison of total NO release from microparticles(500) and microparticles $(2500)$

Figure 3.11 Possible mechanism of SNAP bonding to PLLA by hydrogen bond..............86

Figure 4.1 Preparation of microparticle incorporated chitosan-agarose hydrogel

Figure 5.1 (a) and (b) shows hydrogel-microparticle composite. (c) and (d) shows blank hydrogel without microparticles. (e) and (f) depicts the ascorbic acid chitosanagarose hydrogel.

Figure 5.2 Light initiated total NO release from (a) Hydrogel-microparticles(500) and (b) hydrogel-microparticles $(2500)$ 105

Figure 5.3 comparison of light initiated total NO release rate from Hydrogelmicroparticles(500) and (b) hydrogel-microparticles $(2500)$

Figure 5.4 comparison of total NO release rate from the hydrogel-microparticle $(500)$ and hydrogel-microparticles $(2500)$

Figure 5.5 Illustration of ascorbic acid loaded chitosan agarose hydrogel system used for NO release study in hydrogel-microparticle composite. 108

Figure 5.6 Ascorbic acid initiated total NO release rate from the (a) hydrogelmicroparticle $(500)$ and (b) hydrogel-microparticles $(2500)$

Figure 5.7 comparison of ascorbic acid initiated NO release rate from the hydrogelmicroparticle $(500)$ and hydrogel microparticles $(2500)$.

Figure 5.8 comparison of total NO release from the hydrogel-microparticle $(500)$ and hydrogel-microparticles $(2500)$ 


\section{List of tables}

Table 1 Yield of produced microparticles ...............................................................6

Table 2 Composition (W/O/W), stirring speed, loadings, and particle size distribution and

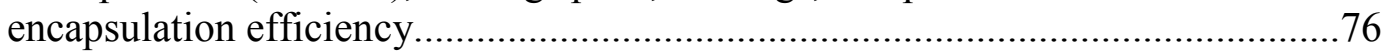

Table 3 Comparison of average total nitric oxide release and encapsulation efficiency from SNAP-PLLA microparticle $(500)$ and SNAP-PLLA microparticle $(2500) \ldots \ldots \ldots . . .86$

Table 4 comparison of average total nitric oxide release from hydrogelmicroparticle(500) and hydrogel-microparticle(2500) by exposure to light and ascorbic acid hydrogel 


\section{Acknowledgements}

I would like to thank my advisor, Dr. Megan Frost for her guidance, support, training and motivation throughout the research work. It was an honor and a privilege to work in the Frost Laboratory which made my research enjoyable and exciting, even at times when research didn't go as planned. I would also like to convey my gratitude to my committee members, Dr. Smitha Rao and Dr. Lanrong Bi for their guidance, help and support.

A special thanks to my family (my parents and younger brother) for believing in me over the years and their patience, and support in making this possible. I would like to dedicate my work to my parents and my brother who have always encouraged me continue pursuing my dreams and motivated me to never give up. I feel honored to say that I would not be here today without their support system. I would also like to thank my friends for their love, encouragement and help during my challenging times. 


\section{List of abbreviations}

\begin{tabular}{|c|c|}
\hline $\mathrm{DCM}$ & Dichloromethane \\
\hline DDD & Drug delivery device \\
\hline EGF & Epidermal Growth Factor \\
\hline $\mathrm{EtOH}$ & Ethanol \\
\hline eNOS & endothelial nitric oxide synthase \\
\hline FGF & Fibroblast growth factor \\
\hline KGF & keratinocyte growth factor \\
\hline NO & Nitric Oxide \\
\hline $\mathrm{O} / \mathrm{W}$ & Oil-in-water solvent evaporation method \\
\hline PBS & Phosphate buffer saline solution \\
\hline PDGF & Platelet derived growth factor \\
\hline PGA & Polyglycolic acid \\
\hline PLA & Polylactic acid \\
\hline PLLA & Poly (L-lactic) acid \\
\hline PLGA & Poly (lactic-co-glycolic) acid \\
\hline PVA & Poly (vinyl alcohol) \\
\hline SNAP & $S$-nitroso- $N$-acetyl-D-penicillamine \\
\hline TGF & Transforming Growth Factor-Beta \\
\hline RPM & Revolutions per minute \\
\hline RT & Room temperature \\
\hline $\mathrm{W} / \mathrm{O} / \mathrm{W}$ & water-in-oil-in-water solvent evaporation method \\
\hline VEGF & vascular endothelial growth factor \\
\hline
\end{tabular}




\begin{abstract}
After injury, wound healing is a complex sequential cascade of events essential for the proper recovery of the wound without the scar formation. Nitric oxide (NO) is a small, endogenous free-radical gas with antimicrobial, vasodilating and growth factor stimulating properties. NO has wide biomedical application especially in wound healing however, its usability is hindered due its administration problem as it is highly unstable.

In this work, poly (1-lactic acid) microparticles encapsulated with NO donor S-nitroso-Nacetyl-D-penicillamine (SNAP) were prepared using water-in-oil-water double emulsion solvent evaporation method for controlled delivery for NO at the specific site. The NO release from SNAP-PLLA microparticles prepared at two different stirring speeds (500 and 2500 RPM) was evaluated using three main SNAP decomposition triggers i.e. light, copper and ascorbic acid. The light controlled on and off mechanism of nitric oxide release from the microparticles at different intensities was demonstrated that can be used to control the amount of NO release depending on the need. An average total NO release of $6.11 \pm 1.71$ $\times 10^{-08}$ for 9 hrs. and $8.43 \pm 2.92 \times 10^{-08}$ for $13 \mathrm{hrs}$. was observed from microparticle $(500)$ and microparticle $(2500)$ respectively on exposure to light, copper (II) and ascorbate. Furthermore, these SNAP-PLLA microparticles were embedded into thermosensitive chitosan-agarose hydrogel to form microparticle-hydrogel composite. These composites have demonstrated NO release on exposure to light. An ascorbic acid chitosan-agarose hydrogel composite system was also used to evaluate the NO release in the presence of only ascorbic acid from the microparticle hydrogel composite. A significant difference in average total NO release from both microparticles as well as microparticle-hydrogel composite was observed because of varying size parameter. These findings suggest that these microparticle as well as microparticle-hydrogel composite formulations may be useful for wound site administration of nitric oxide to accelerate wound healing process delayed due to dysfunction in endogenous NO production caused by pathological infection.
\end{abstract}




\section{Introduction}

Millions of people suffer from acute and chronic wounds around the world annually whose treatments result in major health problems, economic loss, stress, and decreased quality of life of the patient, $(1,2)$. At least $1 \%$ of the population from developed countries will suffer complex chronic wounds during their lifetime and in United States alone more than 6.5 million people suffer from complex wounds (3), costing about $\$ 10$ billion are spent per year managing these wounds. The global wound care market is estimated to reach over $\$ 22$ billion (US) per year by the year 2020 (1). According to a study, wound infection is responsible for $77 \%$ of the surgical deaths (4). The relative risk of death in surgical patients with wound infection is 2.2 times higher than patients without infection. Burgeoning cost due to patient's wound care management have fueled the research in finding cost-effective wound treatments.

The primary objective in the management and treatment of wounds is to attain rapid healing of the skin with pristine functionality. Acute wounds normally heal in the orderly and timely natural process of wound healing, however, not all wounds heal satisfactorily. These chronic wounds present the major economic and ethical problem. Acute wounds heal through the reparative process of healing involving phases of inflammation, migration, proliferation and remodeling following the establishment of new skin layers with its barrier function (Figure 1). On the other side, chronic wounds encounter impaired and delayed healing due to either local factors such as contamination, or complications namely ulcers (diabetic, vascular, ischemic or pressure ulcers). This results in need for more long and careful treatment methods and therapeutic agents for proper recovery of the wound. Recent advances in the knowledge of pathophysiology of wound have led to the development of various techniques such as wound dressing. In the recent years, controlled drug delivery systems have spiked interest in curing the wounds. Several controlled release systems have been developed for direct sustained delivery of the drugs to the wound site.

Controlled Drug Delivery Systems (DDSs) are developed and designed to deliver drugs at target site at preset rates for predefined period. They may be classified into two broad 
categories; targeting and controlled release. Targeted DDSs are the systems which delivers the drugs to the desired tissues and organs while controlled DDSs are the systems controlling the release rate of drug (5). The use of these targeted delivery system is highly beneficial in treatment of chronic wounds as they eliminate the requirement for periodic administration of drugs in conventional dosage forms. Chronic Wound healing requires administration of high doses of antibiotics to control infection as chronic wounds lack sufficient blood supply. Conventionally, drugs are administered systemically into the body which exposes body to the high dose of antibiotics. Instead of systemic administration, localized and prolonged delivery of antibiotic is beneficial. In the form of controlled release system, topical administrations of antimicrobial can be used to avoid side effects of high dose systemic exposure. Topical delivery of the encapsulated active drug also ensures the safety of the drug as the skin never gets in contact with the entire drug at a time (6). In addition, controlled drug delivery systems can be used to provide susceptible drugs such as growth factors which are unstable but in encapsulated form can be stable. Drug release profiles can also be altered and tailored according to the needs of the specific situation. Today, polymeric materials still provide the most important parameters for drug delivery research, primarily because of their ease of processing and the ability of researchers to readily control their chemical and physical properties via molecular synthesis (7).

\subsection{Wound healing process}

The skin is physical barrier present on the human body which provides protection against the infection and diseases. In the event of an injury, a cascade of events is activated to repair the wounded tissue. As shown in the figure 1.1, wound healing is a dynamic process divided into four precise, sequential and regulated stages: (a) hemostasis (b) inflammation (c) proliferation and (d) remodeling $(8,9)$. The ideal wound healing events in a healthy adult are as follows: (a) fast hemostasis; (b) proper inflammation; (c) proliferation and migration of granulation tissue to the healing site; (d) angiogenesis; (e) rapid reepithelialization and (f) strengthen the healing tissue by synthesis and cross-linking of collagen in proper order (10). 
When the injury occurs, the process of hemostasis begins immediately with the vasoconstriction of the broken vessel slowing bleeding. The blood components provide a temporary plug to the wound site and initiate the wound healing cascade. Platelets at the injury site aggregate and interact with the soluble blood proteins and surrounding matrix forming a fibrin clot, and initiating the inflammatory stage (11). The platelets from this clot and the wound tissues release growth factors such as epidermal growth factor (EGF), fibroblast growth factor (FGF), platelet derived growth factor (PDGF) and transforming growth factor-beta (TGF- $\beta$ ) and cytokines. These molecules signals the migration of inflammatory cells to the wound by chemotaxis endorsing the inflammatory stage (12). The first inflammatory mediator on injury site are the neutrophils attracted by the signaling molecules which eliminate the entering microorganisms and cellular debris $(12,13)$. They also release reactive oxygen species (ROS) and proinflammatory cytokines for additional inflammatory cells recruitment $(13,14)$. The mast cells in this stage are responsible for release of vascular endothelial growth factor (VEGF), thus increasing in vascular permeability which is important in cell recruitment from the bloodstream to the wound site (15). They also signal the recruitment of monocyte from the bloodstream by releasing signaling molecules such as TGF- $\beta$, PDGF, FGF, keratinocyte growth factor (KGF), EGF and interleukin 1 (IL-1). Monocytes differentiate into macrophages after migrating from the bloodstream into the wound site where they clear bacteria, debris and apoptotic cells (including neutrophils) helping to resolve inflammation (16). Apart from their phagocytic role, macrophages also produce cytokines and growth factors such as TGF- $\alpha$, TGF- $\beta$, VEGF, FGF and PDGF. The duration of the inflammatory stage vary depending on the size and severity of the wound but in general takes $24 \mathrm{~h}$ to six days after injury to subside (17, 18). The signaling molecules released from the platelets, neutrophils and mast cells in response to injury causes the recruitment of fibroblasts and epidermal cells, thus transitioning into the next stage of wound healing - the proliferative stage. The proliferative stage following the inflammatory stage commences as matrix and tissue cells migrate into the wound site from the periphery. This phase is characterized by reepithelialization which is the proliferation and migration of epithelial cells over the fibrin clot. This phase is characterized by the replacement of fibrin clot with granulation tissue 
(fibroblasts, collagen, and newly formed extracellular matrix (ECM)) (19). In the regenerating injured tissue, endothelial cells and fibroblasts from the intact margins of wound site replicate and migrate into wound and secrete proteoglycans and glycosaminoglycans, the main constituents of ECM such as collagen. Epidermal cells, mainly keratinocytes are stimulated by the KGF released by fibroblasts as well as IL-1 and TNF- $\alpha$ released by macrophages and platelets. They proliferate along the wound edges and create an epithelial bridge as well as accumulate granulation tissue to close the wound below the fibrin clot (9). The cell and tissue proliferation is supported by the growth of blood vessels at the wound site. Keratinocytes release VEGF which stimulate the angiogenesis resulting in migrating of new vessels from surrounding intact capillaries into the wound site. The wound fibroblasts transition into contractile myofibroblasts which work to contract the wound edges together (17). The initial dermal tissue reconstruction begins after 3 to 5 days after initial injury while the proliferation phase can last a few days to several weeks (17). After ECM proliferation, the wound healing is followed by the last stage of wound healing which is remodeling phase. In this stage, the collagen reorganization and maturation occur. The unnecessary collagen and recently formed capillaries are degraded. The granulation tissue transitions into scar tissue, thus healing the wound. The remodeling stage can last from a few weeks to several months or a year (17). The newly closed wound is weaker than the pristine tissue as the tensile strength of scar tissue is less than pre-injured tissue (8). When the wound healing does not proceed by these steps, it results in chronic wounds. Depending on the type and size of the wound, the healing time can differ but no satisfactory reduction in area (20-40\%) after 4 treatment weeks or unhealed wounds up to 4 months are considered to be chronic $(10,20)$.

In all the chronic wounds, a problem in any stage of the normal wound healing mechanism causes impaired healing. Wounds are typically struck in two stages which are inflammatory stage and proliferation stage. Hyperactive inflammatory processes or bacterial infection in the inflammatory stage while insufficient tissue growth and its integration in proliferation stage can become the reason the wound is unable to reach the remodeling phase making it chronic. 


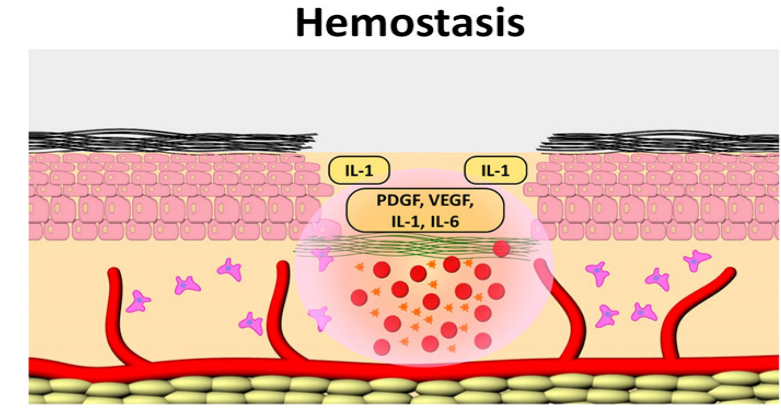

Proliferation

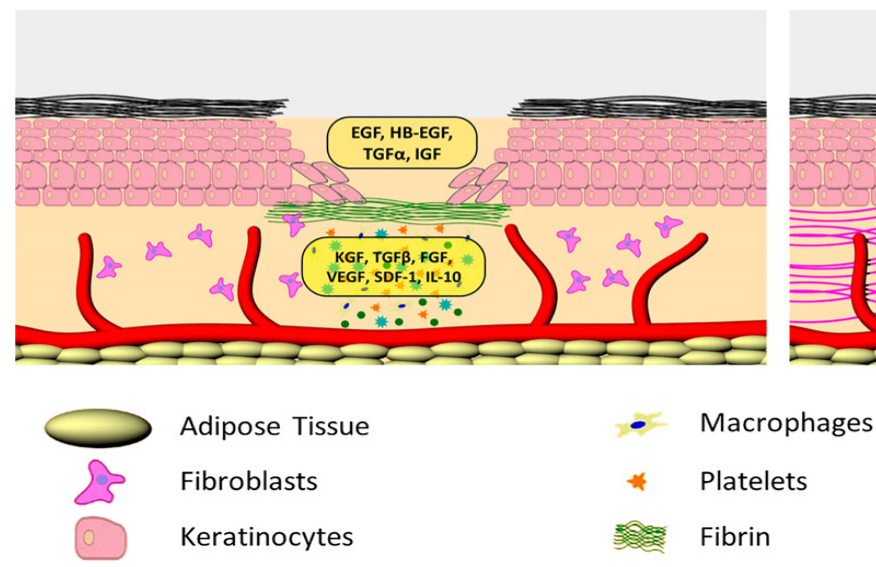

Inflammation

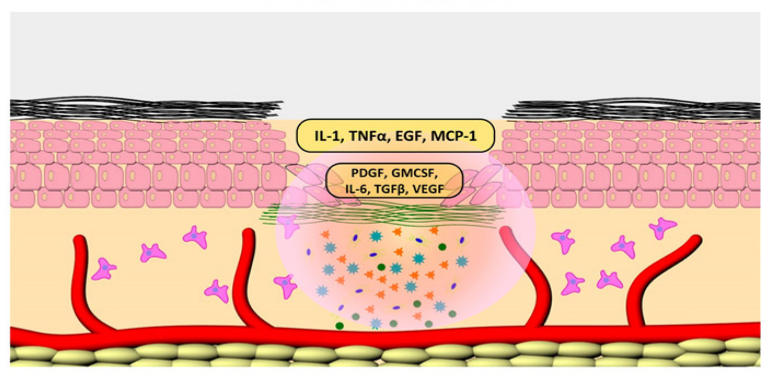

Remodeling

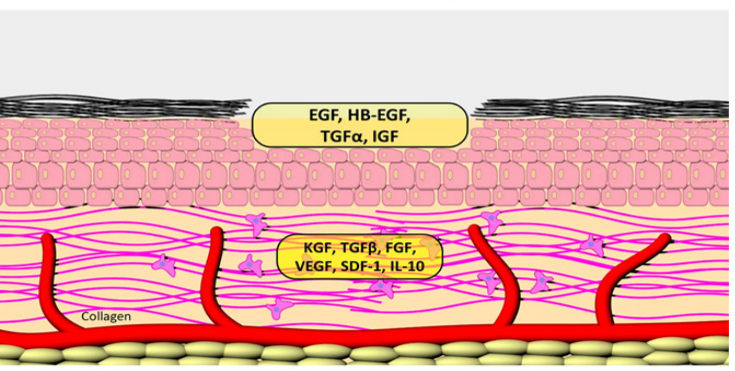

Blood Vessel
$\quad$ Neutrophils
$\quad \begin{aligned} & \text { Pocal and Circulating } \\ & \text { Red Blood Cells }\end{aligned}$

Figure 1.1. Wound healing process (Hamdan. S. et al. 2017) (21) 


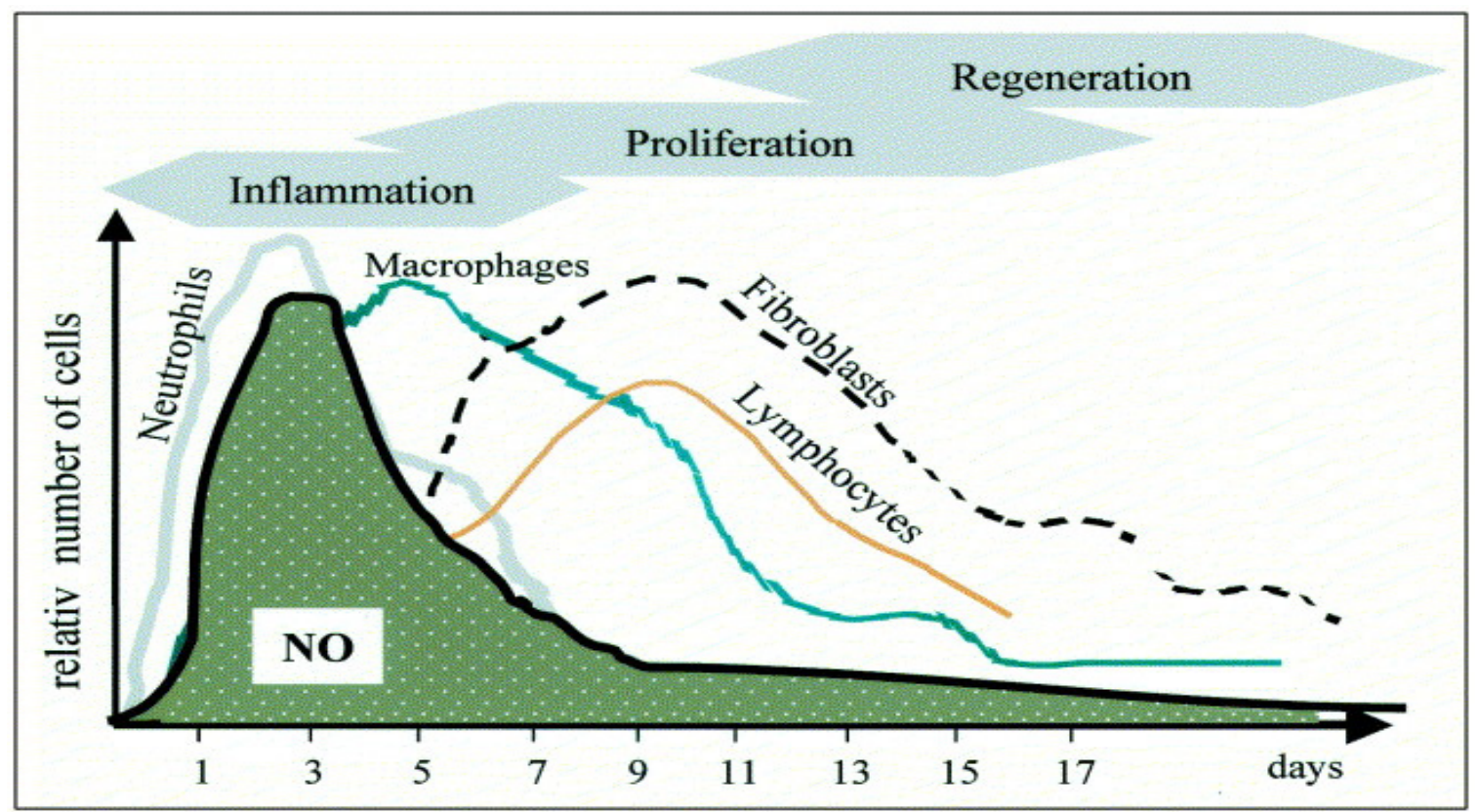

Figure 1.2. Generation of NO during different phases of wound healing (Witte et al. 2002) (22)

\subsubsection{Wound healing complications}

One of the characteristic of chronic wounds is chronic inflammation due to an excessive neutrophil infiltration (8). Although neutrophils are crucial in normal wound healing, their large volume and prolonged presence has adverse effects. The ECM components and wound repair mediators (such as VEGF and PDGF) are destroyed by the enzyme collagenase and elastase released in excessive amounts by neutrophils (23). In addition to this, surrounding tissues are damaged by accumulation of excessive reactive oxygen species in the wound (24). Chronic wounds also show abnormal cell growth as the fibroblasts exhibit low proliferation while keratinocytes at the wound edges showing hyperproliferation. This results in migration blockade of fibroblasts and keratinocytes into the wound $(23,24)$. Low wound fibroblast activity results in decreased angiogenesis and collagen formation. This leads to inadequate granulation tissue formation and decreased blood flow to the wound tissues resulting in insufficient wound closure. All this increases the possibility of tissue breakage as tensile strength of the new tissue is low, resulting in chronic wound and repeating wound opening process (25). 
The inflow of inflammatory cells to the wound site is rapid as they help in clearing tissue debris and bacteria to provide clean environment for wound healing. Although bacterial presence in wound is common, their concentration burden and growth state are critical factors in making a wound chronic. In acute wounds, bacterial infection is prevented by the immune system of the body as the bacteria is in free floating or planktonic state (26). In chronic wounds, the bacterial burden increases so much that the immune system cannot clear the viable bacteria. For example, in a diabetic patient inflammatory phase in wound healing is inhibited, impeding the neutrophils and macrophages migration to the wound. This leads to the decrease in cytokine release as well as low adherence and phagocytosis causing inefficient bacterial targeting (27). If bacteria remain un-checked in the body, it has the tendency to attach to wound surface, accumulate and excrete polymeric matrix forming a biofilm (28). At the outset, the platonic bacteria attach to the surface reversibly and is susceptible to antibiotics. After initial attachment, within hours it becomes irreversibly adhered to the surface, multiplying and secreting polymeric matrix, exopolysaccharide (EPS). Bacteria form microcolonies in the next stage and continue to secrete EPS causing the formation of thick film (29). A mature biofilm develops as the bacteria form column like structure due to the film growth. Bacteria begin to detach from the mature biofilm and spread elsewhere. Penetrating the EPS is harder than destroying individual bacteria cells which makes bacterial biofilm targeting a challenge for the immune system (30). The inflammatory response is elevated when the body recognizes the persistent presence of bacterial biofilm but is ineffective against the biofilm resulting to chronic inflammation along with the infection $(28,30,31)$. A broad variety of bacterial strains such as gram-positive Staphylococcus aureus (S. aureus) and Enterococcus faecalis (E. faecalis) as well as gram negative Pseudomonas aeruginosa and Escherichia coli (E. coli) are found to colonize chronic wounds and lead to biofilm development. Recently, a common bacterium gram negative Acinetobacter baumannii (A. baumannii) has emerged infecting wounded soldiers in Iraq and Afghanistan and is now widespread throughout the U.S hospitals (32-34). Antibiotics have been used widely for treatment of both acute and chronic bacterial infections, even though not effective equally against both of them (31). Antibiotics are effective against free floating easily accessible planktonic bacteria, but lost 
effectiveness against thick matrix of biofilms. They can target dividing planktonic bacteria but are unable to penetrate the biofilm, thus rendered ineffective against dormant bacteria in the biofilms (26). Despite their inefficacy, antibiotics are still largely used to treat bacterial infections where biofilms are present but another more effective method is required to treat chronic wounds.

\subsection{Nitric oxide}

The free radical gas nitric oxide (NO) is a small endogenous molecule involved in various biological functions. It acts as a chemical messenger as it can readily diffuse through cell membranes especially in the cells of vascular endothelium, immune and neural systems (35). It acts as a key mediator in cellular communication affecting the functions of these systems. It also plays important role in regulating processes such as cellular apoptosis and differentiation (36). NO was once known only as a dangerous pollutant generated by industries and automotive acting as a potential carcinogen $(37,38)$. However, the discovery of its synthesis in mammalian cells and its vital role in the physiological processes attracted a lot of medical and scientific interest (39). This led to a rapid increase in NO research (40). Now being one of the important mediators of intracellular as well as extracellular process, NO has become a major target of the pharmaceutical industry (41).

Endogenous NO is quite unstable having half-life of 1-5 $\mathrm{s}$ in physiological environments and rapidly reacts with biomolecules. Endogenous NO is produced by nitric oxide synthase (NOS) enzymatically by oxidizing $L$-arginine to $L$-citrulline. The three isoforms of NOS: endothelial nitric oxide synthase (eNOS), neuronal nitric oxide synthase (nNOS), and inducible nitric oxide synthase (iNOS) are present depending on the site. The NO generated by these three isoforms differs in their expression pattern and regulation (42). While eNOS and nNOS are calcium dependent and constitutively expressed producing low concentrations for short period, on the other hand iNOS is calcium independent which

produces high amount of NO for longer period (43). As a response to inflammatory cytokines, iNOS stimulates NO release in epithelial, endothelial and inflammatory cells. NO can help in the diagnosis of various human diseases such as cardiovascular, 
neurological, gastrointestinal, renal, genitourinary and even cancer (38). There are various specific functions of nitric oxide, out of which some major is: vascular tone maintenance by vasodilation, monocyte and neutrophil adhesion inhibitor to vascular endothelium, inflammation regulator, antioxidative property, neurotransmission regulator, platelet function regulator (40). Meanwhile, addition to these benefits NO is potentially a toxic agent. During oxidative stress, $\mathrm{O}_{2}$ intermediates during $\mathrm{NO}$ generation which can lead to antioxidant deficiency (41).

High NO production by iNOS induced by inflammatory cytokines lead to formation of reactive oxygen and nitrogen species resulting in cellular apoptosis (44). The physiological activity of NO is concentration dependent. Higher concentration of NO can lead to cytotoxicity overcoming the protective mechanisms of the cell (45).

\subsubsection{Role of nitric oxide in wound healing}

Figure 1.2 demonstrates the generation of nitric oxide during the different phases of wound healing process. The production of NO peaks during the inflammatory stage and diminishes toward the proliferative stage (22). After the skin injury, iNOS is elevated throughout the inflammation, granulation tissue formation and re-epithelialization which makes iNOS the most relevant NOS isoform to the wound healing process. During the inflammatory stage, iNOS in neutrophils and macrophages is stimulated to produce nitric oxide by cytokines (such as TNF- $\alpha$ and IL-1) (46-49). NO stimulates the vasodilation of the microvasculature to increase the blood flow in the wound and aid inflow of inflammatory cells from the bloodstream (22). The inflammatory stage is dominated by neutrophils and macrophages while in the proliferative stage fibroblasts are the most active cells responsible for collagen deposition at the wound site. Wound site fibroblasts have NO releasing capabilities while fibroblasts in un-injured skin tissue do not. NO inhibition in vivo has shown to impair wound healing due to decrease in wound collagen production $(50,51)$. NO is also produced by the intact keratincytes near the wound stimulating more keratinocyte proliferation and migration in the wound (48). 
During bacterial infection, iNOS production is upregulated from the macrophages due to signals from cytokines and bacterial lipopolysaccharides (LPS) which in turn stimulates the NO production from L-arginine. NO doesn't have significant direct bactericidal activity but acts as an intermediate and is part of non-specific immune response. NO helps in bactericidal action by reacting with the oxygen and oxygen radicals which form reactive byproducts. NO levels in the healthy tissues are low but during inflammatory conditions, free radical, reactive oxygen species such as superoxide $\left(\mathrm{O}_{2}-\right)$ are produced by macrophages and neutrophils through NADPH oxidase enzymatic action (44). NO and superoxide interact to form peroxinitrite (ONOO-) which is strongly antimicrobial. It induces oxidative stress on DNA nucleotides in bacteria leading to nucleotide cleavage and cell apoptosis $(52,53)$. Overexpression of iNOS causes high NO production which reacts with oxygen species such as superoxide and other biomolecules resulting in the formation of powerful oxidizing reactive oxygen and nitrogen species. These cause protein oxidation, DNA base deamination, impaired cellular function, lipid peroxidation, increased inflammation, enzymes nitrosylation and cell apoptosis (54).

Eukaryotic cells are also susceptible to damage by NO but requires a higher concentration than the level that damages bacterial cells because of protective mechanisms that scavenge nitrogen oxygen species (44). In the presence of bacteria products and oxidative stress, upregulation of thiol protein - metallothionein in eukaryotic cells acts as a protective mechanism. The thiol on the protein react with the reactive NO byproducts thus reducing intracellular levels of harmful reactive species $(55,56)$. This clearly shows that NO plays a significant role in wound healing which is why utilizing NO in the treatment of chronic wounds can prove extremely beneficial.

In the past few decades, a lot of research has been done to harness and release NO for the treatment of wounds. The extended use of NO is unlikely to show any bacterial resistance as compared to traditional antibiotics and alternatives such as silver (57). The therapeutic effects of different techniques such as gaseous release, small molecule and macromolecular NO delivery have been studied. The delivery method, concentration and time frame are among the several factors which determine the efficiency of the providing 
$\mathrm{NO}$ as a therapeutic agent in the wound healing. The simplest path to test NO's effect on wound is by administering the molecule in its gas phase (gaseous NO). Although gaseous $\mathrm{NO}$ is relatively easy to administer, difficulty in patient mobility due to equipment size (NO cylinder) and toxic effect of large quantity of NO limits its usage and require for sophisticated method.

\subsubsection{NO donors and their application}

To overcome the limitation in delivery of gaseous NO, material systems incorporated with NO donors have been studied and developed. The main goal is to develop a material capable of delivering controlled, predictable levels of NO under physiological conditions over a given period. The difficulty in non-systemic route of administration of NO is also a major challenge for administration of exogenous NO. For this reason, NO donors have been used widely which mimic the endogenous NO production by NOS. NO donors are functional moieties or small drugs that can generate NO exogenously when administered (58). There are many classical low molecular weight NO donors which are used, out of which four major widely used NO donors are diazeniumdiolates (NONOates), Snitrosothiols (RSNOs), metal-nitrosyl complexes, and NO drug hybrids (58). Jones et al. utilized probiotic NO-releasing patches in which immobilized Lactobacilli ferment glucose into lactic acid (anaerobic reaction) which in turn reacts to sodium nitrite to produce $\mathrm{NO}(\mathrm{g})$ topically diffusing freely through the adhesive. It may be a safe and cost-effective method as it showed accelerated wound healing in ischemic wounds of New Zealand's white rabbit (59).

The process of blending small molecule NO donor into a polymer or synthesizing the polymer along with the covalently attached NO donor are the primary method of producing NO from biomaterials. Among various potential NO donors, some compounds have proven useful for this purpose. Organic nitrates (e.g. nitroglycerin) and nitrites require the metabolic activation by the enzymes such as mitochondrial aldehyde dehydrogenase (mtALDH), even with this required conversion, they are widely used NO prodrugs (60). 
Their incorporation in polymer matrices restrict their bioactivation which makes them somewhat difficult to use.

Topical administration of acidified nitrate cream which generate NO via ascorbic acid reduction of nitrite to $\mathrm{NO}$ found to accelerate wound healing in normal and diabetic mice (61). It can be useful for targeted NO delivery due to its ease of administration, however, its use is limited because of cutaneous inflammation and erythema caused at the application site.

One of the interesting classes of NO releasing molecules for wound healing is diazeniumdiolates (NONOates) because of their ease of synthesis and predictable, controlled NO release in aqueous environments. Synthetic NO donors are formed via the reaction between NO and a variety of different amine groups. Shabani et al. applied a polyethylenimine cellulose NONOate polymer (PEIC-NO) topically to dermal wounds of rats showing an accelerated wound closure (62). Additionally, a significant increased collagen production is observed when human dermal fibroblasts were cultured with a polyvinyl NONO-ate hydrogel wound dressing. In diabetic mice model these hydrogel platforms exhibited thicker granulation and scar tissue but no significant increase in wound closure time (63). In another study, poly (lactic-co-glycolic acid)-polyethylenimine (PEI) nanoparticles (NO/PPNPs) incorporated with NONOate were developed which showed extended NO release for over 6 days (64). They were found to have a propensity to bind to negatively charged methicillin-resistant staphylococcus aureus (MRSA) and P. aeruginosa surface due to positive charge of nanoparticles. This showed bactericidal efficacy of nanoparticles without any toxic effect to fibroblast cells and favorable wound healing in MRSA-infected full-thickness wounds. Regardless of their advantages, potential of carcinogenic nitrosamine formation due to NONOate decomposition products interaction hinders the clinically use of many NONOates.

\subsubsection{S-Nitrosothiols}

S-Nitrosothiols (RSNOs) are thiol derived endogenously produced class of NO-releasing compounds. S-Nitroso-N-acetylpenicillamine (SNAP) is an example of an RSNO shown 
in Fig 1.3. These NO donors (RSNO) are known to release NO through thermal, photolytic, and transition metal decomposition pathways (65). RSNOs can be characterized by UVVis spectroscopy. The primary RSNO group exhibit a $\pi \rightarrow \pi^{*}$ transition centered near 340 $\mathrm{nm}$ (major peak). A less significant second smaller peak due to the $\mathrm{n} \rightarrow \pi^{*}$ transition occurs near $550 \mathrm{~nm}$ in primary and secondary RSNOs (66). RSNOs display either a red/pink (primary and secondary) or green (tertiary) color because of the visible range absorbance. The copper (metal) ion mediated and ascorbic acid mediated decomposition are the two mechanisms of NO release from RSNO. $\mathrm{Cu}+$ decompose the RSNO into nitric oxide and the corresponding disulfide (65).

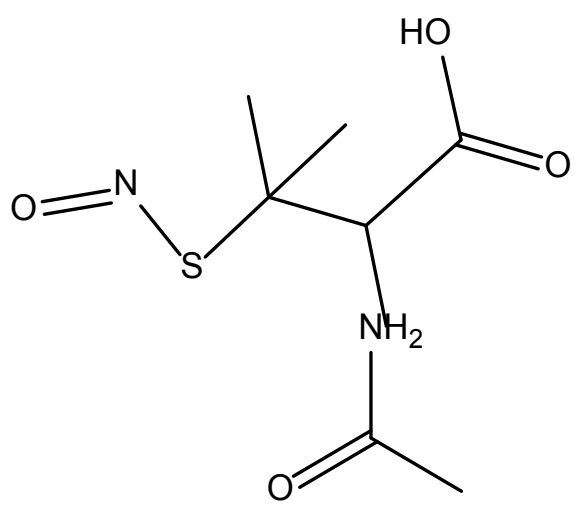

Figure 1.3. S-Nitroso-N-acetylpenicillamine (SNAP)

S-nitrosothiols react with ascorbic acid in two reactions depending on the latter's concentration.

At low concentration, ascorbate acts as a reducing agent which generates $\mathrm{Cu}^{+}$from $\mathrm{Cu}^{+2}$, thus decomposing RSNOs into nitric oxide and the corresponding disulfide, whereas at higher ascorbate concentration, the reaction is independent of $\mathrm{Cu}^{+2}$ and products formed are nitric oxide and the RSNO derived thiol (66). Another pathway for NO release from RSNOs is via photo initiated decomposition. This is a crucial factor as light can act as an on/off switch to generate NO in a controllable manner whenever needed. This avoids the need of other NO release triggering mechanisms (67). 


\section{$2 \mathrm{RSNO} \rightarrow 2 \mathrm{NO}+\mathrm{RSSR}$}

Frost et al. showed photo initiated $\mathrm{NO}$ release from a tri-layer silicon rubber film in which one layer blended with S-Nitroso-N-acetyl-D,L-penicillamine derivatized fumed silica was sandwiched between two layers of pure silicon rubber without need of $\mathrm{Cu}$ ion and ascorbate. Intensity and duration of the light exposed to the film determined the amount of NO generated (68). Gierke et al., covalently linked a S-Nitroso-N-acetyl-D-penicillamine to polydimethylsiloxane and developed a photo initiated NO releasing polymer material (SNAP-PDMS) (69). A number of RSNO based NO releasing biomaterials have been proposed for wound healing application with antithrombotic and antibacterial activity.

\subsection{Microparticles drug delivery system}

\subsubsection{General}

Several NO releasing materials such as polymeric nanoparticles, dendrimers, inorganic nanoparticles (metallic nanoparticles, silica nanoparticles, liposomes, carbon nanotubes and quantum dots have been described in different research articles $(40,70-75)$. Their synthesis, characterization and application for NO delivery have been thoroughly studied. However, their use in the wound healing is still a relatively novel approach which has yet to be explored.

Microparticles (also known as microspheres) are the small, spherical particulate system with the diameter ranging in the size of $1 \mu \mathrm{m}-1000 \mu \mathrm{m}$. They have been proven ideal in providing sustained and controlled release. In recent years, polymeric microparticles have gained a great deal of interest in the field of drug delivery because of their well-defined production parameters to obtain desired size ranges (76). The freedom to control the dimension of the microparticles makes their injectability easier. They hold a wonderful opportunity due to their drug reservoir and bioactive molecule carrier ability as well as their controlled drug release characteristics. The release from the particles can be achieved either by degradation of the polymer matrix or leaching of the drug from the particles (77). 
Different biodegradable carrier matrices have been used for the microparticle production (synthetic or natural) in therapeutics delivery (78). Natural polymers such as collagen, chitosan, alginate and proteins used in microparticle production undergo enzymatic degradation while hydrolytic degradation happens in synthetic polymers such as polylactic acid (PLA), polycaprolactone (PCL), polyglycolic acid (PGA) and polylactic-co-glycolic acid (PLGA).

Microparticles are an attractive drug delivery strategy for a variety of drugs including proteins, peptides, hydrophilic and hydrophobic small molecules. Alteration in the polymer weight, hydrophilicity and particle size can directly control the release of the therapeutic molecule carrying by the microparticle. Microparticle protects the encapsulated drug against degradation, offers controlled and local delivery over periods and simple administration compared to alternatives such as macro sized implants. Polymeric systems can incorporate hydrophilic and lipophilic drugs with relatively high efficiency. A drug can be homogeneously distributed throughout the polymer matrix (microparticles) or form a drug reservoir by encapsulating into polymer (microcapsules). These properties make microparticle drug carriers potentially tunable in controlling dose and rate of drug delivery.

\subsubsection{Different preparation methods of polymeric microparticles}

Microparticles are usually a homogenous mixture comprised of two main components: polymer and active agent. The core of the microparticle may be solid, liquid, or even gas (79). There are various methods employed for preparation of microparticles for different biomedical applications. The employed method used to encapsulate the drug in the polymer matrix should meet the following requirements $(80,81)$ :

1) The processing parameters of the microparticle fabrication must not affect the stability and biological activity of the drug.

2) The encapsulation efficiency of the drug and yield of the microparticle should be high enough and in the desired range respectively. 
3) The microparticle characteristics (such as quality and the drug release profile) should be highly reproducible.

Some of the basic polymeric microparticle fabrication methods are described below:

\subsubsection{Coacervation (precipitation by non-solvent addition)}

In this method solid crystals or aqueous solution of the drug is dispersed in an organic polymer solution following an addition of second organic solvent (non-solvent) in which polymer is insoluble thus causing phase separation. A phase inducer (e.g. mineral oil) is added to induce coacervation. Due to insolubility of hydrophilic drugs, high encapsulation efficiency can be obtained by this method, however high cost of handling oils, high microparticle aggregate formation and difficult to control parameters makes it less promising. The parameters which affect the outcome of this method are the drug: polymer ratio, the polymer solvent, the process temperature, stirring rate or the non-solvent volume or type.

\subsubsection{Emulsion salting out}

In this technique, polymer is dispersed in water miscible organic solvent along with the drug. This drug containing polymeric organic solution is emulsified into an electrolyte (magnesium acetate, calcium chloride, sodium chloride, magnesium chloride) saturated aqueous solution (82). A stable oil in water $(\mathrm{O} / \mathrm{W})$ emulsion is produced due to the salting out effect which prevents mixing of polymeric organic solvent with an aqueous phase saturated with electrolytes. An excessive amount of water is added to the emulsion which induces reverse salting out effect leading to the formation of microparticles as the water miscible solvent diffuses into aqueous phase from the emulsion droplets. The dissolution of polymer in nonhalogenated solvents and no requirement for high energy/pressure device for emulsification are advantages of this method, however, there is concern about the recycling of massive quantities of salt used can be a problem. 


\subsubsection{Spray drying}

In spray drying, organic polymer solution along with suspended drug is spray dried to form the microparticles. Compared to $\mathrm{O} / \mathrm{W}$ emulsion evaporation technique (unsuitable for hydrophilic drugs), spray drying can incorporate both hydrophilic and hydrophobic drugs in the microparticles. The organic solvent used in spray drying should evaporate quickly in the drying phase so solvents such as methylene chloride or ethyl acetate should be used to dissolve polymer. For example, NO donor containing inhalable PLGA microparticles produced by spray drying have been shown to target and control release of NO to the macrophage for killing intracellular Mycobacterium tuberculosis (83). But there are few draw backs of this method such as formation of needle shaped crystals due to incompatibility of drug and polymer, and fibers formation due to insufficient dispersion force which break up polymer solution (84).

\subsubsection{Solvent partitioning precipitation}

In solvent partitioning, a drug containing polymer in organic solvent solution is slowly injected into a stream of mineral oil or vegetable oil. The drug and the polymer coprecipitate as the organic solvent is soluble in oil while drug and polymer are not which led to their partitioning in oil. The solubility of the drug is a crucial factor in this method. The drug will precipitate along with polymer if it is soluble in polymer solution while the polymer will precipitate around solid drug if it suspended in solution. The size of the microparticles in this method are affected by the drug: polymer ratio, diameter of the needle, choice of organic solvent and flow rate of the mineral oil (85).

\subsubsection{Supercritical fluid extraction method}

Supercritical fluids are the substances which are above their critical point $(T>T c)$. These show gaseous flow properties and liquid's dissolving power. These have no surface tension which make them easily penetrable through the materials. At critical point they experience changes in their density which relates to their solvent power which can be controlled by 
temperature and pressure. (86). There are two major two techniques used in SCF. The first is to dissolve the drug and polymer in the SCF and then fast expansion of supercritical solution (RESS) following spraying the melt forming particles. The second is to melt the drug and polymer in SCF and then precipitating the particles from gas saturated solution (PGSS) after releasing the gas (87). This method requires special equipment and therefore are not widely used on the bench scale.

\subsubsection{Solvent evaporation method}

The solvent evaporation method has been widely explored for the polymeric microparticle preparation in delivering different drugs $(80,87,88)$. There are several variable parameters which affect the properties of the microparticles that are drug solubility, solvent type, polymer composition (type, viscosity and molecular weight), diffusion rate, temperature, stirring rate, internal morphology and drug loading $(81,84,87,89)$. The successful entrapment of the drug into the microparticles defines the productiveness of this method to produce microspheres. The drugs which are either insoluble or poorly soluble in the continuous phase (i.e. the aqueous medium) have been most successfully encapsulated using this process (90). Several types of drugs and active agents have been formulated into polymeric systems, including nitric oxide releasing agents $(74,91,92)$. Different solvent evaporation techniques are available for microparticle preparation depending on the hydrophobicity or the hydrophilicity of the drug for efficient drug encapsulation.

\subsection{Single emulsion}

Single emulsion process involves oil-in-water $(\mathrm{O} / \mathrm{W})$ emulsification. In this system, polymer and drug (to be encapsulated) are dissolved in a volatile solvent (i.e., organic phase) and emulsified in a surfactant containing aqueous phase. This method is suitable and frequently used for encapsulating insoluble or poorly water-soluble and lipid soluble drugs. 
This is the simplest method among all emulsion techniques and other methods are variation of the single emulsion. This system involves four major steps: (a) polymer and hydrophobic drug are dissolved in an organic solvent; (b) organic phase, called dispersed phase, is emulsified in an aqueous phase called continuous phase resulting formation of droplets; (c) solvent is extracted from dispersed phase by solvent evaporation, thus hardening droplets into particles and (d) microparticles are recovered and dried by eliminating the residual solvent.

In most systems to prepare microparticles using oil in water emulsions, the components necessary are organic phase (polymer and drug dissolved in a volatile solvent), a surfactant dissolved in an aqueous phase. This organic and aqueous phase are mixed to form emulsion $(\mathrm{O} / \mathrm{W})$ by appropriate stirring using a propeller or a magnetic bar at suitable temperature conditions. A surfactant is amphipathic in nature and is added into the aqueous phase to stabilize the formed droplets and prevent them from coalescing and microparticle flocculation. Surfactants align at the droplet surface and lowers the free energy at the interface between the two phases, thus promote their stability. One of the widely used surfactant is poly vinyl alcohol (PVA).

After the emulsion formation, solvent removal is done by either evaporation or extraction to harden the droplets. The solvent removal by evaporation involves maintaining the emulsion at a reduced or atmospheric pressure to evaporate the volatile solvent. This results in the evaporation of the organic solvent at the water-air interface after its leaching out of the droplet into aqueous phase. On the other hand, in extraction, the emulsion is added to a large quantity of water which causes the organic solvent to diffuse out of the oil droplets. The extraction method depends on the following factors: the temperature of medium/water, emulsion volume to extraction medium ratio and the solubility of the polymer, solvent and the dispersed phase. Extraction method is faster than evaporation process, however the microparticles made by extraction are more porous which can lead to undesirable drug release profiles. The formation of particles with a high porosity will occur due to high extraction leading to undesirable drug release profiles (93). 
The major disadvantage of $\mathrm{O} / \mathrm{W}$ emulsification process is the poor encapsulation of the hydrophilic drugs. One reasons can be the hydrophilic drug not dissolving in the organic solvent. Other being, hydrophilic drug diffusing or partitioning out from the dispersed organic phase into the continuous aqueous phase resulting in loss of the drug and deposition of microcrystalline fragments of the drug onto the microsphere surface which causes initial burst release of the drug (81). Four alternative methods have been described and employed to increase encapsulation of hydrophilic drugs. These methods are water-inoil-water $(\mathrm{W} / \mathrm{O} / \mathrm{W})$ emulsion, o/w co-solvent method, oil in oil $(\mathrm{O} / \mathrm{O})$ emulsion, and waterin-oil (W/O) emulsion (94).

In the co-solvent system, the drug (which is not soluble in one organic solvent) is dissolved in a second solvent (co-solvent). In oil in oil $(\mathrm{O} / \mathrm{O})$ emulsion method, the drug can be dissolved or suspended in oil phase and then emulsified in another oil phase (87). Another method known as water-in-oil (W/O) emulsion is also employed in which polymer and drug are solubilized in water-miscible organic solvent (such as acetonitrile) and then dispersed in oil following the evaporation of organic solvent from dispersed phase and washing off the oil by solvents (such as n-hexane) (80).

\subsection{Double emulsion}

For hydrophilic or drugs insoluble in organic solvent, a double emulsion process is used. One method which can be employed is solid-in-oil-in-water emulsion $(\mathrm{S} / \mathrm{O} / \mathrm{W})$ process provided the size of the drug form is small $(1-10 \mu \mathrm{m})$. A very low drug particle size is necessary for high encapsulation in desired microparticles. Both hydrophilic drugs (cisplatin) and hydrophobic drugs (87) have been encapsulated using this method. The number of drug properties including parameters affecting this method are properties of the drug crystal such as their hardness, elasticity, melting point, hygroscopicity, and decomposition due to thermal or light sensitivity. A smooth mortar and pestle can be used for a non-hygroscopic drug while a ball mill apparatus can be used for hygroscopic drugs. However, there are certain drawbacks of this method such as ball mill is low efficient in terms of drug recovery, drug might get contaminated while size reduction process (ex. wear 
and tear of the ball), drug might show sedimentation (higher density) or flotation (low wettability) (87).

Another method to encapsulate hydrophilic drugs is water-in-oil in water $(\mathrm{W} / \mathrm{O} / \mathrm{W})$ emulsion process. In this process, firstly a $\mathrm{W} 1 / \mathrm{O}$ primary emulsion is formed by mixing an aqueous drug solution (W1) to an organic solvent containing polymer (organic phase) along with the stirring or sonication. This primary emulsion is then dispersed in another surfactant containing aqueous phase (W2) to form the $\mathrm{W} 1 / \mathrm{O} / \mathrm{W} 2$ emulsion. This method is widely used to encapsulate peptides and proteins at high encapsulation efficiency (87). The main drawback of this method is stabilization of the inner emulsion which can result in the loss of drug containing aqueous droplets to the external aqueous phase (W2). A surfactant that dissolve in the organic solvent (such as sorbitan) can be used to stabilize inner emulsion.

\subsection{Microparticles preparation by solvent evaporation method}

\subsubsection{Materials}

\subsubsection{Dispersed phase}

\subsection{Polymer}

One of the major deciding factor in encapsulating a therapeutic agent in microparticle is the polymer used. Different polymer parameters such as type of polymer, molecular weight, composition (homopolymer or copolymer), concentration and hydrophilicity influence the encapsulation of drug in microparticle. The type of polymer and its degradation properties affect the drug release rates. Degradable polymers can be categorized in two groups depending on the rate of their functional group hydrolysis: surface (heterogeneous) eroding and bulk eroding (homogenous) (95). Surface eroding polymers (ex. polyanhydrides and poly (ortho esters)) degrade from the surface only as they are composed of hydrophobic monomers while bulk eroding polymers (ex. PLA, PLGA) degrade throughout the polymer matrix. Surface eroding polymers degrade into 
oligomers and monomers at polymer-water interface as water is not able to penetrate the polymer matrix whereas in bulk eroding polymers water permeates through polymer and degrades throughout the matrix. Surface eroding polymers are fast degrading compared to bulk eroding polymer due to their composition (more hydrophobic monomers) (95).

Polymer molecular weight, concentration and hydrophilicity affect the degradation rate, encapsulation efficiency and drug release rates. Usually, high polymer weight and concentration show increased encapsulation efficiency and low initial burst release. Increase in hydrophilicity increases the degradation rate of polymer and high release rate (76).

\subsection{Solvent}

An acceptable solvent required for the solvent evaporation technique should meet following criteria: ability to dissolve the polymer, poor solubility in continuous phase, highly volatile and low boiling point, low toxicity (94). Chloroform has been used frequently as a solvent but has been replaced with dichloromethane due to former's toxicity and low vapor pressure. Dichloromethane shows high volatility, poor miscibility with water and low boiling point which has made it the most common solvent for solvent evaporation technique. However, due to carcinogenic nature of dichloromethane, less toxic solvents such as ethyl acetate have also been studied as a potential substitute. Although less toxic, ethyl acetate shows partial miscibility in water (4.5 times higher compared to dichloromethane) which decreases the encapsulation efficiency of microparticles and are non-uniform. So, currently dichloromethane is the most used solvent because of fast evaporation, high drug encapsulation efficiency and more spherical and uniform form microparticles (94).

\subsubsection{Continuous phase}

\subsection{Surfactant}

Surfactants are used in the solvent evaporation method to stabilization of the dispersed phase in the continuous phase. It stabilizes the emulsion by reducing the surface tension of 
the continuous phase, thus avoiding the coalescence and agglomeration of the dispersed droplets. They are the amphiphilic in nature and are chosen based on polarity of both immiscible phases, microparticle desired size distribution and sphericity (94). In literature surfactants are divided into four types depending on the nature of their hydrophilic molecule part: anionic, cationic, amphoteric and non-ionic. For ex. PVA (polyvinyl alcohol) is the most used surfactant. The microparticle size decreases with the increase in surfactant concentration as it lowers surface tension of continuous phase until critical micelle concentration $(\mathrm{CMC})$ is reached. Surface tension does not decreases after reaching critical micelle concentration (CMC) (94).

\subsubsection{Operating conditions}

\subsubsection{Agitation}

Agitation is an important parameter for controlling the microparticle size. Li et al. (2008) also reported agitation to be one of the most important parameter for controlling the microparticle size only next to the physicochemical properties of materials. Factors such as container geometry; position, number and size of impeller; impeller to container diameter ratio determines the size distribution of the droplets formed in the emulsion. The increase in agitation rate decrease the average microparticle size (94).

\subsubsection{Temperature and pressure}

The evaporation rate of the solvent during the microparticle formation can be accelerated either by increasing the temperature of the continuous phase or by reducing the reactor pressure. However, elevated temperature can result in lower yield, larger size distribution, lower drug encapsulation efficiency and coarser morphology (96). It can result in denaturation or degradation of the drug. Applying reduced pressure can be an option as it increases encapsulation efficiency. But, it is not easy and lowering pressure compared to the saturated vapor pressure of the solvent at a given temperature can boil the solvent (94). 


\subsection{Poly (l-lactic acid)}

Biodegradable polymers have gained wide use in biomedical and pharmaceutical industry. A suitable biodegradable polymer would have following properties:

(1) Metabolization and elimination from the body by normal physiological pathways

(2) Easy fabrication

(3) Degradation into non-toxic substances

(4) No inflammatory reaction after application

(5) Degradation product of the biodegradable polymer should be carbon dioxide and water.

According to these criteria, polylactide (PLA) and their co-polymers are considered as suitable biodegradable polymers. PLA has asymmetric $\alpha$-carbon which can be described as d- or 1- stereochemical centers (or R or S, respectively) resulting in two enantiomeric forms of poly D-lactic acid (PDLA) and poly L-lactic acid (PLLA). PLLA is crystalline or semi crystalline while PDLA is completely amorphous due to disordered polymer chains. Fig 1.4 shows the structure of PLLA. PLLA has melting temperature (56) of approximately $150^{\circ} \mathrm{C}$ and a glass transition temperature $(\mathrm{Tg})$ of approximately $50^{\circ} \mathrm{C}$. Due to crystallinity, PLLA is less permeable compared to the amorphous polymers. Crystallinity of PLLA also gives its mechanical strength and stiffness properties. Poly (l-lactic acid) (PLLA) has been widely studied for drug delivery because of its biodegradability and biocompatibility. Molecular weight of the polymer is directly related to polymer chain size which affects the degradation rate. High molecular weight polymers require more time to degrade. However, for PLLA this effect is opposite because of inverse relation of crystallinity with molecular weight. PLLA which is pure polymer is likely to sustain the drug release for longer period due to its longer degradation half-life (97). 


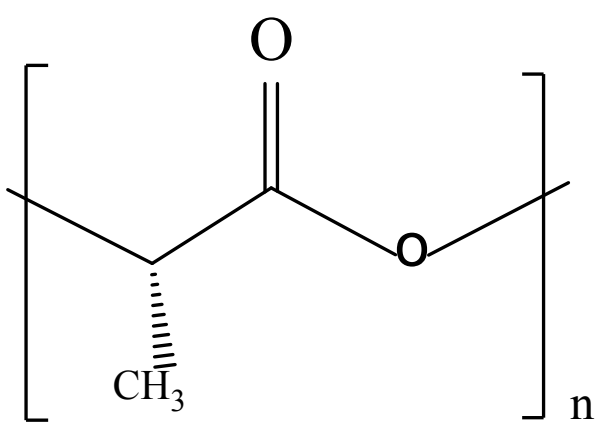

Figure 1.4 Structure of PLLA

\subsubsection{Drug release from PLA devices}

Polymer degradation is the breakdown of polymer chains into oligomers and monomers resulting in decrease in molecular weight after degradation. After degradation, erosion occurs in the polymer which is the loss of material from the polymer bulk resulting in weight loss.

The drug release from the PLA devices occur using two main mechanisms: diffusion and degradation/erosion. At first the drug release is diffusion controlled and at the end it is degradation/erosion controlled. Drug release from the PLA device starts with the water absorption following hydrolysis and erosion. The drug release profile of PLA device degradation is mostly tri-phasic, sometimes bi-phasic which are first order release kinetics and rarely mono-phasic which is zero-order release kinetics. Tri-phasic profile is observed usually in large particles as heterogeneous degradation occurs inside the particles while biphasic release profile is observed in smaller particles. Thus, alteration of release profile can be done by using different sizes of particles (98).

There are three distinct phases of drug release: burst release, slow release and second burst. In the first phase i.e. burst release free, non-encapsulated drug from and/or near the surface of the particles is released. It can also happen due to the crack formation or disintegration of the device. The burst release results in faster drug release in the medium immediately after exposure which decreases the lifetime of the device. It is usually an undesirable 
negative phenomenon. However, initial burst is sometimes desirable such as in cutaneous wound healing which can provide immediate relief followed by prolonged release to promote gradual healing $(99,100)$. The main disadvantages of the burst release are unpredictability and uncontrollable amount of drug release. It may be controlled or avoided by different methods $(76,87)$.

In the second phase, also called lag-phase drug diffusion occurs slowly through the polymer or the pores due to polymer hydration. It is slow release phase and degradation of the polymer occurs simultaneously (98). Third phase also known as second burst is fast release phase which marks the beginning of polymer erosion. In this phase, drug is now released by both diffusion and erosion as polymer is hydrolyzed to oligomers and monomers. Evidently, not all the drug release profiles follow the traditional tri-phasic release profile. In cases of rapid, short or absence of second release phase, release may slow down at the end. Several factors affect the drug release profile which make release profile prediction very difficult.

\subsection{Hydrogel}

Hydrogels are hydrophilic three-dimensional, and crosslinked polymer networks that can absorb significant amount of water or biological fluid up to $90 \%$ of their dry weight. They are soft and elastic material usually used above their glass transition temperature. They have the capability to absorb water attributed to the presence of hydrophilic side groups (hydroxyl, carboxyl, amide and sulfonic groups) connected to the polymer backbone (101). Crosslinks present in the hydrophilic hydrogel make them insoluble in aqueous environment to maintain their structure. Because of their structural (compositionally and mechanically) similarity to the living tissue (extracellular matrix) and ability to promote cell adhesion and proliferation, the hydrogels have gained wide interest in the field of tissue engineering and drug delivery (101). 


\subsubsection{Classification of hydrogels}

Hydrogels can be classified based on a variety of their characteristics such as nature of material, nature of side groups, mechanical and structural features, method of preparation, physical structure, crosslinking and their responsiveness to the physiological environment stimuli (101).

The simplest way to classify the hydrogel is based on origin of polymer: natural or synthetic (101). Natural polymers are obtained from recyclable material such plants and animals. The hydrogel of natural polymer is biocompatible, biodegradable and have bioactive site in their structure but mechanically weak compared to synthetic polymers. Whereas the synthetic hydrogels have well known structures which enable their modification for required properties. Some examples of natural polymers include agarose, alginate, chitosan, fibrin, collagen and hyaluronic acid while Synthetic polymers include poly (ethylene glycol), poly (ethylene oxide), polyvinyl alcohol, polyacrylates and polypeptides $(101)$.

Based on the nature of side group, the hydrogel can be classified as neutral, cationic, anionic or ampholytic. On the basis of preparation method, hydrogel may compose of one type of monomer (homopolymer), two types of monomers (copolymer), and more than three types of polymer (multipolymer). One type of polymer can be synthesized or crosslinked in the presence of another inflated polymer network (101). At Least one type of polymer must be hydrophilic in each type.

Based on the configuration, hydrogels are classified on the basis of physical and chemical structure which are amorphous, semi crystalline and crystalline (101).

Classified on the basis of cross linking hydrogels are divided in two categories: chemically cross-linked hydrogels and physically cross-linked hydrogels chemical crosslinking means covalent bond formation between different functional groups of polymers (101). It can be done by different methods such as free radical polymerization, high energy irradiation, enzymes use and complementary groups' chemical reaction. Physically cross-linked 
hydrogels are usually the biodegradable hydrogels which do not need any external crosslinking agent. Cross-linking agents can be toxic and non-biodegradable and often requires their residual removal before their use in the biomedical industry. Highly ordered and assembled chain segments in polymer are held together by secondary molecular forces such as hydrogen bonding, Van der Waal forces or hydrophobic interactions which keep together their amorphous hydrophilic phase (101).

Another way to classify hydrogel is to divide them based on their responsiveness to the external environment stimuli. Stimuli responsive hydrogels are stimuli sensitive that are able to sense and express varying degree of response to even minor changes in the external environment conditions such as physical stimuli (temperature, electric or magnetic field, light, pressure, and sound) or the chemical stimuli ( $\mathrm{pH}$, solvent composition, ionic strength, and molecular species).

Temperature responsive hydrogels: These exhibit temperature dependent sol-gel transition Tgel, which correlate to the lower critical solution temperature (LCST), and gel-sol transition temperature Tp (upper critical solution temperature, UCST), which correlates to dissipation or precipitation of a gel (102). The $\mathrm{H} 2 \mathrm{O}$ molecules bonds with the polar groups of the polymer through hydrogen bonding at temperature below LCST. With the increase in temperature above LCST, these polymer network collapse as the hydrogen bonds release to the bulk leading to large gain in entropy. Some examples of thermoresponsive hydrogels are hydrogels containing polymers such as chitosan PEG-poly, N-isopropylacrylamide hydrogel (PNIPAA), methyl cellulose. They can have useful applications in sustained drug delivery and tissue engineering (103).

$\mathrm{pH}$ responsive hydrogels: The functional group of a polymer in $\mathrm{pH}$ responsive hydrogel is added to a weak acidic group (ex. acrylic acid) or weak basic group (ex. amine). It results in sudden swelling of the polymer due to change in $\mathrm{pKA}$ and $\mathrm{pH}$. Some polymers have carboxylic acid as functional groups which accept hydrogen at low $\mathrm{pH}$. They exchange carboxylic ion for cation above $\mathrm{pKA}$ value and become ionized at higher $\mathrm{pH}$. As the 
carboxyl groups gets ionized, the volume and swelling capacity of these polymers increase till it reaches a plateau near $\mathrm{pH} 7$ (102).

\subsubsection{Hydrogels in wound therapy}

The hydrogel structure can have structural similarity to ECM due to their high water content (104). They have a key role in tissue engineering as they can help in cellular recognition by acting as an artificial ECM. A hydrogel must meet certain criteria to promote new tissue formation which include physical, biological and biocompatibility parameters.

Hydrogel can be real helpful in the treatment of the wound. They are appropriate for cleaning a dry, sloughy or necrotic wound through dead tissue rehydration. They can increase autolytic debridement and provide coolness to the wound surface leading to a noticeable pain reduction and increasing patient acceptability. Hydrogels do not adhere to the wound if required can be easily removed due to their chemical properties. The hydrogels which fulfill all these parameters are suitable to be used as 'ideal dressing' (105). Some commercially available hydrogels for skin regeneration are TegadermTM Hydrogel Wound Filler (3M), DermagelTM Hydrogel Sheets (Medline Industries), and AquaDermTM Hydrogel Sheet (DermaRite).

Hydrogels can be used as drug delivery systems which can be used for controlled delivery and release of bioactive molecules into the wound (106). In majority of cases, Slow release profile of bioactive molecules is desirable to assure their presence during the required whole period of healing. For this objective, some researchers have incorporated growth factors (VEGF, FGF etc.) in the hydrogel. These growth factors are released along the entire wound healing process and are important in the process. Topical delivery of FGF using glycosaminoglycan hydrogel by Liu et al. demonstrated its potential in compromised wound healing patients (107). Hydrogels can be incorporated with RGD sequences similar to natural ECM to enhance cellular recognition of ECM and to promote cell adhesion (108). Due to their properties, hydrogels are a good potential candidate for protein/drug release as they are able to maintain protein structure and drug activity. The rate of release from 
the 3D matrix of the hydrogel is generally diffusion controlled through aqueous channels within hydrogels (109). Therefore, it is difficult to control the release of protein/drug over a long time using hydrogel. To overcome this limitation, different strategies such as microparticle incorporation in the hydrogel have been studied (110). As shown in Fig 1.5, microparticles can be encapsulated with the drug can be embedded in the hydrogel.

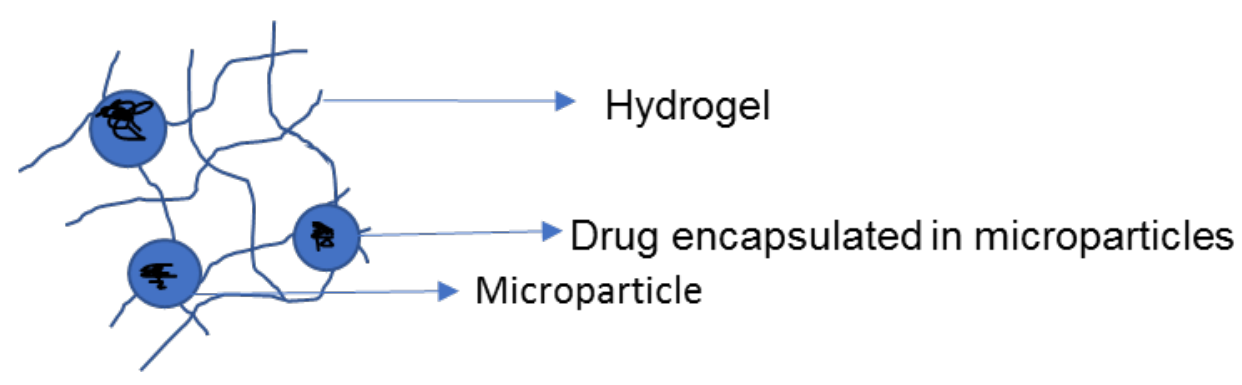

Figure 1.5 Microparticle encapsulation in hydrogel

Microparticles, nanoparticles and liposomes have been studied and developed as controlled drug release systems. Therefore, these carriers can be incorporated in the hydrogel to avoid a burst release of bioactive molecules from hydrogel. The carrier entrapment in the hydrogel network enhances the efficiency of bioactive molecules release in vivo at the targeted site. Moreover, hydrogel support the carrier, increases stability of the bioactive molecule within carrier and provide an extra diffusion barrier for drug release which increases the drug release period. Alongside, it allows the scaling down of therapeutic dose administered to the patient (111). Bae et al. developed a sustained drug delivery system by incorporating PLGA microparticles a sustained release system for local drug delivery that incorporated model drug (5-fluorouracil) loaded PLGA microparticles into thermosensitive chitosan hydrogel. This system demonstrated a decrease in the drug burst release and was 
proposed to be used as an injectable drug carrier system (112). After considering the excellent properties and sustained bioactive molecule release applications of hydrogel in wound healing, these systems will be further studied for its future application in biomedical and clinical industry.

\subsubsection{Chitosan}

Chitosan is a linear polymer of $\beta$-(1-4) linked D-glucosamine residues and N-acetyl-Dglucosamine groups (Fig 1.6). It is a natural, semi crystalline and positively-charged polysaccharide made by deacetylation of chitin, a component present in the exoskeleton of crustaceans. The major parameters affecting the chemical characteristics of chitosan are its molecular weight and degree of acetylation. Depending on degree of deacetylation, chitosan is commercially available in low molecular weight, medium molecular weight and high molecular weight (113). When using chitosan as a dressing for wound treatment degree of deacetylation is a crucial factor.

The size of Chitosan polymer molecules is large. The water holding capacity of high molecular weight (HMW) chitosan in a hydrogel is more compared to a low molecular weight (LMW). Chitosan molecules can form bonds or interaction with other molecules as the chitosan deacetylation results in appearance of amino groups. Amino groups have an antimicrobial effect. Therefore, degree of deacetylation is a key factor in anticipating the extent of wound healing acceleration by the hydrogel. Chitosan with high degree of deacetylation can be a desired parameter for gel preparation for wound treatment (114). Chitosan is insoluble in inorganics and at neutral $\mathrm{pH}$ but is soluble in weak acids such as acetic acid (115).

Chitosan can aid to wound healing as it stimulates hemostasis and accelerates tissue regeneration. A natural product is often preferred over a synthetic material for biomedical research due to its biocompatibility. Chitosan is biodegradable as it can be metabolized by human enzymes (such as lysozyme) and is non-toxic. The chitosan has been approved by FDA in the USA for use in wound dressings $(74,75)$. Chitosan preparations are shown to be toxic to bacteria, fungi and parasites. Its structural similarity to glycosaminoglycan's 
and hydrophilic nature makes it an attractive material for a tissue engineering scaffold. Nacetylglucosamine is an anti-inflammatory drug which is monomeric unit of chitosan can help in wound repair.

Chitosan may provide a cool and soothing effect when applied to an open wound inducing an analgesic effect. When applied as a topical agent on open wound such as skin burns and ulcers, excellent pain relief was observed by chitosan (70). Due to anti-inflammatory effects of chitosan, these are beneficial for the treatment of prolonged inflammation at the wound site. Water-soluble chitosan has shown to significantly suppress the expression and secretion of proinflammatory cytokines Water-soluble chitosan significantly suppresses the secretion and expression of proinflammatory cytokines (tumor necrosis factor- $\alpha$ and interleukin-6) and inducible nitric oxide synthase in astrocytes (70).

Chitosan has an antimicrobial activity due to presence of its cationic nature. The cationic amino groups of chitosan bind to the anionic groups of the bacteria causing the inhibition of growth of these bacteria which can be useful during the infectious period of a burn wound, as bacterial infection may delay the healing. This positively charged nature of chitosan due to cations also facilitate the effective cell growth and protein attachment. Ribeiro et al. developed and studied the chitosan hydrogel wound dressing on the isolated fibroblast cells from rat skin to assess the cytotoxicity of the hydrogel. The results displayed cell adhesion and proliferation was promoted by the chitosan hydrogel and cell viability studies showed non-cytotoxic nature of hydrogel and its degradation byproducts. This suggested that chitosan hydrogel may help in skin regeneration (116). Both either blending or crosslinking of chitosan with other polymers are effective procedure to enhance its physical and mechanical properties along with expanding its area of application (117).

It could also form polyelectrolyte complexes with various natural and synthetic polymers which can have various applications in biomedical (drug delivery) and medicine industry (118). Many altered chitosan hydrogels exhibit thermosensitive characteristics. For example, Bhattaraim et al. developed a copolymer by grafting PEG onto the chitosan which 
resulted in a thermally reversible hydrogel with LCST of $34^{\circ} \mathrm{C}$. This injectable thermogel was studied for in vitro drug release of model protein bovine serum albumin (119).

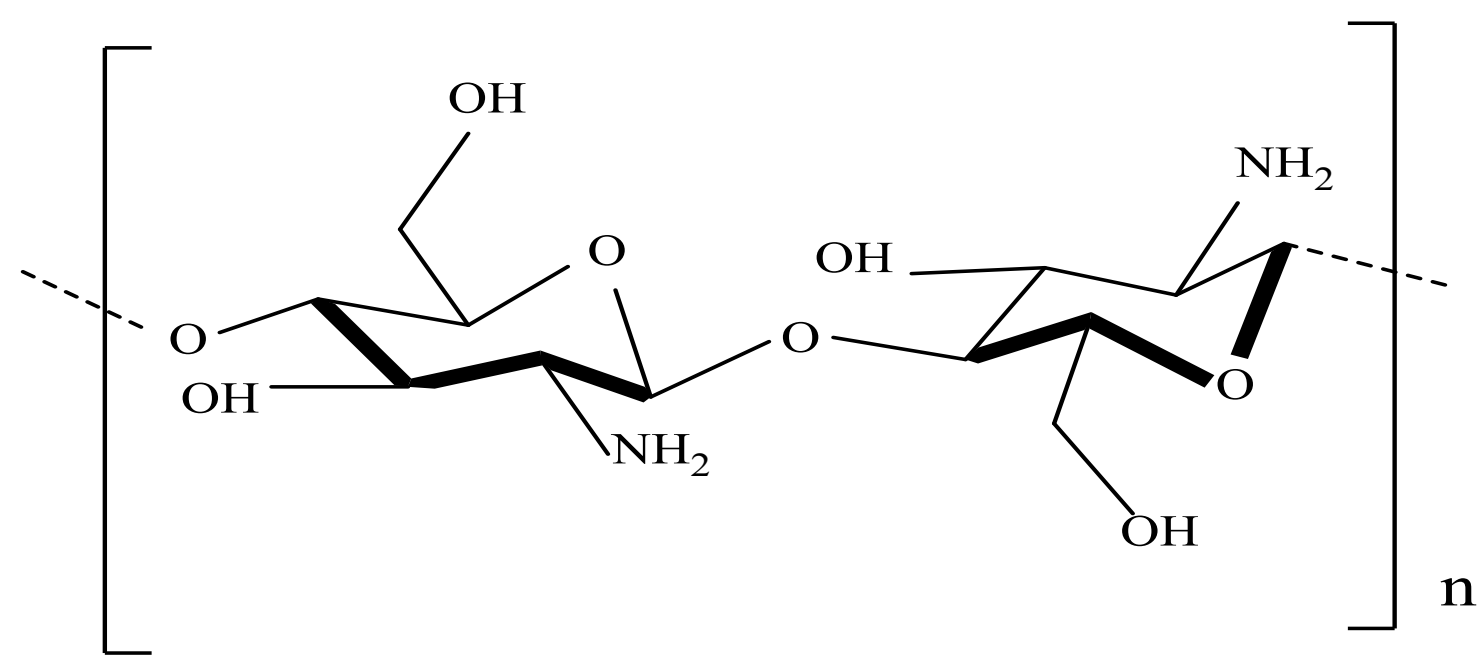

Figure 1.6 Structure of Chitosan

\subsubsection{Agarose}

Agarose is a natural polysaccharide consist of repeating units of alternating $\beta$ (1-4)-linked D-galactose and $\alpha$ (1-3)-linked 3, 6 anhydro-L-galactose residues. It is a neutral polysaccharide obtained from marine red algae (e.g. Rhodophyta). Agarose exhibit LCST at $35^{\circ} \mathrm{C}$ similarly to other temperature sensitive hydrogels. The agarose hydrogel can be hydrophilic, hydrated and swollen below LCST while it collapse, gets hydrophobic and dehydrated (120). The process of gelation is fully reversible without any change in polymer properties.

Agarose can undergo coil-helix transition which makes agarose hydrogels concentration and temperature. The agarose structure becomes more ordered due to conversion of coils to helices through hydrogen bonds. It also affects the mechanical properties of the hydrogel 
(121). Agarose undergo thermoreversible gelation in water environment. Agarose chains are in random coil state at elevated temperature and assemble themselves in left handed dual helices as temperature decreases due to hydrogen bonding and Van der Waal interactions. This helices network becomes more stable and rigid because of further aggregation due to long range interactions leading to hydrogel formation. Again, heating destroys the helices in the hydrogel causing the random coil chain formation.

The agarose polymer concentration, $\mathrm{pH}$ and solvent type also affects the thermal and mechanical properties of the agarose hydrogel. The biocompatibility and capacity of agarose to form very strong gels, even at low concentrations, make it suitable for Tissue Engineering applications. Agarose has shown to support non-immunogenic neurite extension and widely studied in nerve tissue regeneration applications. However, agarose hydrogel show very low cell adhesion and slow degradation rate (122). According to numerous studies agarose itself does not contain cell adhesive amino acid which results in agarose hydrogels not supporting enough cell adhesiveness and proliferation (123). To overcome this limitation, agarose can be modified by either chemically functionalizing with the peptides containing cell recognition motif such as RGD or combining with other polysaccharides. A chitosan-agarose composite hydrogel system developed by GómezMascaraque et al. displayed cell adhesion and proliferation (124). Another composite hydrogel (hyaluronic acid-agarose) developed by Zhang et al. exhibited potential for wound healing (125).

To overcome these limitations, agarose can be chemically functionalized or combined with other polysaccharides $(122,124,125)$. Gómez-Mascaraque et al. developed a hydrogel (chitosan/agarose) that allowed cell adhesion and proliferation $(122,124,125)$. Zhang and collaborators combined hyaluronic acid with agarose and produced a hydrogel that revealed a great potential to improve the wound healing (125). 


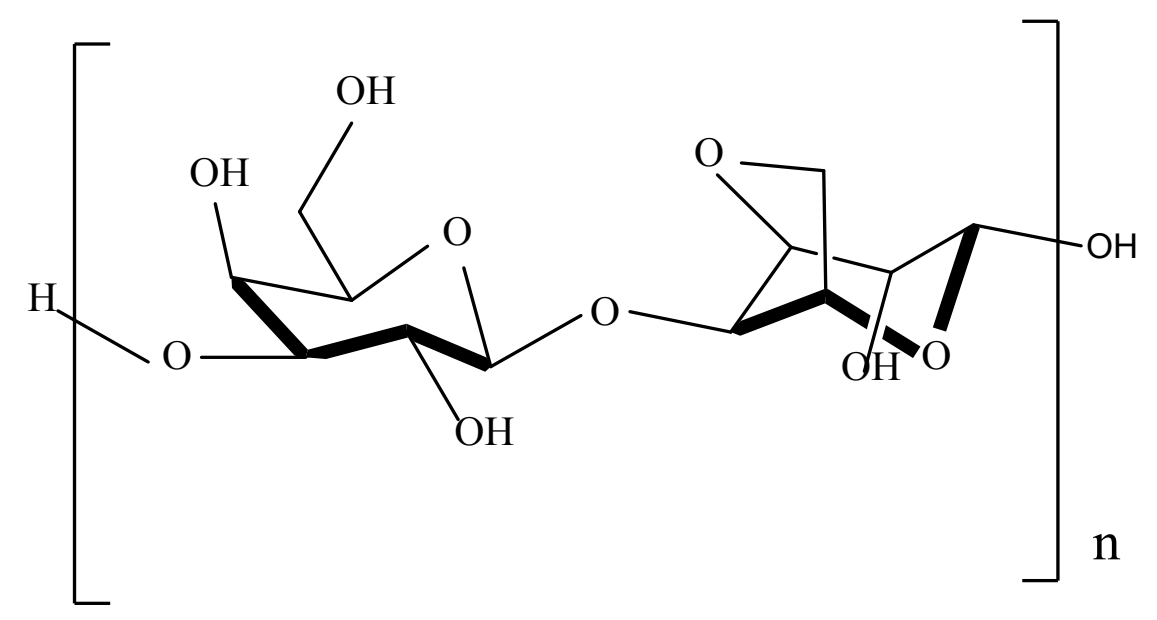

Figure 1.7 Structure of Agarose

\subsection{Statement of Purpose}

Several NO releasing polymer systems have been reported in the literature with promising results for potential biomedical and pharmaceutical applications. Despite their potential benefits, none of the NO releasing material reported to date have been clinically applied. Some of the main problems with these materials are: 1) short durations of NO release 2) production of toxic byproducts 3 ) instability of the NO donor during storage 4) poor dosage control 5) exorbitant cost of $\mathrm{NO}$ donor, and 6) lack of efficient local and systemic delivery. The purpose of developing NO delivering polymer system, particularly in wound healing, is to mimic the antimicrobial role of NO released by the macrophages, endothelial cells and other cells. For this purpose, it is extremely crucial to develop materials which can deliver similar physiologically relevant levels of $\mathrm{NO}$ without risk of any toxic byproduct.

The primary purpose of this thesis research is to develop a simple, cost effective, novel polymer system which can address some of the major challenges listed above. A double emulsion solvent evaporation method was employed to produce poly (1-lactic) acid (PLLA) microparticles encapsulating NO donor S-nitroso-N-acetylpenicillamine (SNAP). The 
resultant microparticles were shown to release NO in a controllable manner with photo initiated. In the presence of copper (II) ions and ascorbate, the microparticles were shown to have instantaneous NO release. Two distinct size of microparticles were produced using two different stirring rates to study differences in their NO loading and NO release profile.

The SNAP loaded NO releasing microparticles were incorporated into a thermosensitive chitosan-agarose hydrogel system. In wound healing, the hydrogel can provide a moist environment in addition to promoting cell growth while NO releasing microparticles can provide antibacterial effect. Therefore, the combination of hydrogel and NO releasing microparticle was studied. The NO release profile of the hydrogel-microparticle composite was studied. The composite was tested for NO release using photoinitiation. Another chitosan-agarose hydrogel containing ascorbic acid was tested to initiate NO release from this hydrogel-microparticle composite. This system may be beneficial for the treatment of chronic wounds.

\subsection{References}

1. Tricco AC, Cogo E, Isaranuwatchai W, Khan PA, Sanmugalingham G, Antony J, et al. A systematic review of cost-effectiveness analyses of complex wound interventions reveals optimal treatments for specific wound types. BMC medicine. 2015;13(1):90.

2. Werner S, Grose R. Regulation of wound healing by growth factors and cytokines. Physiological reviews. 2003;83(3):835-70.

3. Gottrup F. A specialized wound-healing center concept: importance of a multidisciplinary department structure and surgical treatment facilities in the treatment of chronic wounds. The American journal of surgery. 2004;187(5):S38S43.

4. Jan WA, Khan SM, Jehanzeb M, Muazzam M. Surgical site infection and pattern of antibiotic use in a tertiary care hospital in Peshawar. Journal of Ayub Medical College Abbottabad. 2010;22(3):141-5.

5. Putnam D, Kopeček J. Polymer conjugates with anticancer activity. Biopolymers Ii: Springer; 1995. p. 55-123. 
6. Gutowska A, Bark JS, Kwon IC, Bae YH, Cha Y, Kim SW. Squeezing hydrogels for controlled oral drug delivery. Journal of Controlled Release. 1997;48(2):141-8.

7. Vogelson CT. Advances in drug delivery systems. Modern drug discovery. 2001;4(4):49-50.

8. Diegelmann RF, Evans MC. Wound healing: an overview of acute, fibrotic and delayed healing. Front Biosci. 2004;9(1):283-9.

9. Li J, Chen J, Kirsner R. Pathophysiology of acute wound healing. Clinics in dermatology. 2007;25(1):9-18.

10. Siddiqui AR, Bernstein JM. Chronic wound infection: facts and controversies. Clinics in dermatology. 2010;28(5):519-26.

11. Midwood KS, Williams LV, Schwarzbauer JE. Tissue repair and the dynamics of the extracellular matrix. The international journal of biochemistry \& cell biology. 2004;36(6):1031-7.

12. Martin P. Wound healing--aiming for perfect skin regeneration. Science. 1997;276(5309):75-81.

13. Tschaikowsky K, Sittl R, Braun G, Hering W, Rügheimer E. Increased fMet-LeuPhe receptor expression and altered superoxide production of neutrophil granulocytes in septic and posttraumatic patients. Journal of Molecular Medicine. 1993;72(1):18-25.

14. Singer AJ, Clark RA. Cutaneous wound healing. New England journal of medicine. 1999;341(10):738-46.

15. Wulff BC, Wilgus TA. Mast cell activity in the healing wound: more than meets the eye? Experimental dermatology. 2013;22(8):507-10.

16. Delavary BM, van der Veer WM, van Egmond M, Niessen FB, Beelen RH. Macrophages in skin injury and repair. Immunobiology. 2011;216(7):753-62.

17. Baum CL, Arpey CJ. Normal cutaneous wound healing: clinical correlation with cellular and molecular events. Dermatologic surgery. 2005;31(6):674-86.

18. Macri L, Clark R. Tissue engineering for cutaneous wounds: selecting the proper time and space for growth factors, cells and the extracellular matrix. Skin pharmacology and physiology. 2009;22(2):83-93.

19. Yamaguchi Y, Yoshikawa K. Cutaneous wound healing: an update. The Journal of dermatology. 2001;28(10):521-34. 
20. Lazarus GS, Cooper DM, Knighton DR, Margolis DJ, Pecoraro RE, Rodeheaver $\mathrm{G}$, et al. Definitions and guidelines for assessment of wounds and evaluation of healing. Archives of dermatology. 1994;130(4):489-93.

21. Hamdan S, Pastar I, Drakulich S, Dikici E, Tomic-Canic M, Deo S, et al. Nanotechnology-Driven Therapeutic Interventions in Wound Healing: Potential Uses and Applications. ACS central science. 2017;3(3):163-75.

22. Witte MB, Barbul A. Role of nitric oxide in wound repair. The American Journal of Surgery. 2002;183(4):406-12.

23. Demidova-Rice TN, Hamblin MR, Herman IM. Acute and impaired wound healing: pathophysiology and current methods for drug delivery, part 1: normal and chronic wounds: biology, causes, and approaches to care. Advances in skin \& wound care. 2012;25(7):304.

24. Greaves NS, Iqbal SA, Baguneid M, Bayat A. The role of skin substitutes in the management of chronic cutaneous wounds. Wound repair and regeneration. 2013;21(2):194-210.

25. Guo Sa, DiPietro LA. Factors affecting wound healing. Journal of dental research. 2010;89(3):219-29.

26. Hall-Stoodley L, Costerton JW, Stoodley P. Bacterial biofilms: from the natural environment to infectious diseases. Nature reviews microbiology. 2004;2(2):95108 .

27. Hatanaka E, Monteagudo P, Marrocos M, Campa A. Neutrophils and monocytes as potentially important sources of proinflammatory cytokines in diabetes. Clinical \& Experimental Immunology. 2006;146(3):443-7.

28. Seth AK, Geringer MR, Hong SJ, Leung KP, Mustoe TA, Galiano RD. In vivo modeling of biofilm-infected wounds: a review. Journal of surgical research. 2012;178(1):330-8.

29. Høiby N, Ciofu O, Johansen HK, Song Z-j, Moser C, Jensen PØ, et al. The clinical impact of bacterial biofilms. International journal of oral science. 2011;3(2):55-65.

30. James GA, Swogger E, Wolcott R, Secor P, Sestrich J, Costerton JW, et al. Biofilms in chronic wounds. Wound Repair and regeneration. 2008;16(1):37-44.

31. Costerton JW, Stewart PS, Greenberg EP. Bacterial biofilms: a common cause of persistent infections. Science. 1999;284(5418):1318-22. 
32. Eliopoulos GM, Maragakis LL, Perl TM. Acinetobacter baumannii: epidemiology, antimicrobial resistance, and treatment options. Clinical infectious diseases. 2008;46(8):1254-63.

33. Eveillard M, Joly-Guillou M. Emerging Acinetobacter baumannii infections and factors favouring their occurrence. Pathologie-biologie. 2012;60(5):314-9.

34. Peleg AY, Seifert H, Paterson DL. Acinetobacter baumannii: emergence of a successful pathogen. Clinical microbiology reviews. 2008;21(3):538-82.

35. Burke AJ, Sullivan FJ, Giles FJ, Glynn SA. The yin and yang of nitric oxide in cancer progression. Carcinogenesis. 2013;34(3):503-12.

36. Butler A. The biological roles of nitric oxide. Chemistry and industry. 1995(20):828-30.

37. Dusse LMSA, Vieira LM, Carvalho MdG. Nitric oxide revision. Jornal Brasileiro de Patologia e Medicina Laboratorial. 2003;39(4):343-50.

38. Scatena R, Bottoni P, Pontoglio A, Giardina B. Pharmacological modulation of nitric oxide release: new pharmacological perspectives, potential benefits and risks. Current medicinal chemistry. 2010;17(1):61-73.

39. Friedman A, Friedman J. New biomaterials for the sustained release of nitric oxide: past, present and future. Expert opinion on drug delivery. 2009;6(10):1113-22.

40. Saraiva J, Marotta-Oliveira SS, Cicillini SA, Eloy JdO, Marchetti JM. Nanocarriers for nitric oxide delivery. Journal of drug delivery. 2011;2011.

41. Ignarro LJ. Nitric oxide: biology and pathobiology: Academic press; 2000.

42. Bonavida B, Khineche S, Huerta-Yepez S, Garbán H. Therapeutic potential of nitric oxide in cancer. Drug resistance updates. 2006;9(3):157-73.

43. Koehler JJ, Zhao J, Jedlicka SS, Porterfield DM, Rickus JL. Compartmentalized nanocomposite for dynamic nitric oxide release. The Journal of Physical Chemistry B. 2008;112(47):15086-93.

44. Wink DA, Mitchell JB. Chemical biology of nitric oxide: insights into regulatory, cytotoxic, and cytoprotective mechanisms of nitric oxide. Free Radical Biology and Medicine. 1998;25(4):434-56.

45. Coneski PN, Schoenfisch MH. Nitric oxide release: Part III. Measurement and reporting. Chemical Society Reviews. 2012;41(10):3753-8. 
46. Fang FC, Vazquez-Torres A. Nitric oxide production by human macrophages: there9s NO doubt about it. American Journal of Physiology-Lung Cellular and Molecular Physiology. 2002;282(5):L941-L3.

47. MacMicking J, Xie Q-w, Nathan C. Nitric oxide and macrophage function. Annual review of immunology. 1997;15(1):323-50.

48. Öztürk F, Ermertcan AT. Wound healing: a new approach to the topical wound care. Cutaneous and ocular toxicology. 2011;30(2):92-9.

49. Schwentker A, Vodovotz Y, Weller R, Billiar TR. Nitric oxide and wound repair: role of cytokines? Nitric oxide. 2002;7(1):1-10.

50. Schäffer M, Efron PA, Thornton FJ, Klingel K, Gross SS, Barbul A. Nitric oxide, an autocrine regulator of wound fibroblast synthetic function. The Journal of Immunology. 1997;158(5):2375-81.

51. Stallmeyer B, Kämpfer H, Kolb N, Pfeilschifter J, Frank S. The function of nitric oxide in wound repair: inhibition of inducible nitric oxide-synthase severely impairs wound reepithelialization. Journal of Investigative Dermatology. 1999;113(6):1090-8.

52. Delaney S, Delaney JC, Essigmann JM. Chemical-biological fingerprinting: probing the properties of DNA lesions formed by peroxynitrite. Chemical research in toxicology. 2007;20(11):1718-29.

53. Douki T, Cadet J. Peroxynitrite mediated oxidation of purine bases of nucleosides and isolated DNA. Free radical research. 1996;24(5):369-80.

54. Carpenter AW, Schoenfisch MH. Nitric oxide release: Part II. Therapeutic applications. Chemical Society Reviews. 2012;41(10):3742-52.

55. Jones ML, Ganopolsky JG, Labbé A, Wahl C, Prakash S. Antimicrobial properties of nitric oxide and its application in antimicrobial formulations and medical devices. Applied microbiology and biotechnology. 2010;88(2):401-7.

56. Schwarz MA, Lazo JS, Yalowich JC, Allen WP, Whitmore M, Bergonia HA, et al. Metallothionein protects against the cytotoxic and DNA-damaging effects of nitric oxide. Proceedings of the National Academy of Sciences. 1995;92(10):4452-6.

57. Privett BJ, Broadnax AD, Bauman SJ, Riccio DA, Schoenfisch MH. Examination of bacterial resistance to exogenous nitric oxide. Nitric Oxide. 2012;26(3):169-73.

58. Fontana K, Mutus B. Nitric Oxide-Donating Devices for Topical Applications. Nitric Oxide Donors: Novel Biomedical Applications and Perspectives. 2017:55. 
59. Jones M, Ganopolsky JG, Labbé A, Gilardino M, Wahl C, Martoni C, et al. Novel nitric oxide producing probiotic wound healing patch: preparation and in vivo analysis in a New Zealand white rabbit model of ischaemic and infected wounds. International wound journal. 2012;9(3):330-43.

60. Chen Z, Zhang J, Stamler JS. Identification of the enzymatic mechanism of nitroglycerin bioactivation. Proceedings of the National Academy of Sciences. 2002;99(12):8306-11.

61. Weller R, Finnen MJ. The effects of topical treatment with acidified nitrite on wound healing in normal and diabetic mice. Nitric Oxide. 2006;15(4):395-9.

62. Shabani M, Pulfer SK, Bulgrin JP, Smith DJ. Enhancement of wound repair with a topically applied nitric oxide-releasing polymer. W ound Repair and Regeneration. 1996;4(3):353-62.

63. Masters KSB, Leibovich SJ, Belem P, West JL, Poole-W arren LA. Effects of nitric oxide releasing poly (vinyl alcohol) hydrogel dressings on dermal wound healing in diabetic mice. Wound Repair and regeneration. 2002;10(5):286-94.

64. Nurhasni H, Cao J, Choi M, Kim I, Lee BL, Jung Y, et al. Nitric oxide-releasing poly (lactic-co-glycolic acid)-polyethylenimine nanoparticles for prolonged nitric oxide release, antibacterial efficacy, and in vivo wound healing activity. International journal of nanomedicine. 2015;10:3065.

65. Singh RJ, Hogg N, Joseph J, Kalyanaraman B. Mechanism of nitric oxide release from S-nitrosothiols. Journal of Biological Chemistry. 1996;271(31):18596-603.

66. Holmes AJ, Williams DLH. Reaction of ascorbic acid with S-nitrosothiols: clear evidence for two distinct reaction pathways. Journal of the Chemical Society, Perkin Transactions 2. 2000(8):1639-44.

67. Williams DLH. The chemistry of S-nitrosothiols. Accounts of chemical research. 1999;32(10):869-76.

68. Frost MC, Meyerhoff ME. Controlled photoinitiated release of nitric oxide from polymer films containing S-nitroso-N-acetyl-DL-penicillamine derivatized fumed silica filler. Journal of the American Chemical Society. 2004;126(5):1348-9.

69. Gierke GE, Nielsen M, Frost MC. S-Nitroso-N-acetyl-D-penicillamine covalently linked to polydimethylsiloxane (SNAP-PDMS) for use as a controlled photoinitiated nitric oxide release polymer. Science and technology of advanced materials. 2011;12(5):055007.

70. Ahmed S, Ikram S. Chitosan based scaffolds and their applications in wound healing. Achievements in the Life Sciences. 2016;10(1):27-37. 
71. Duan S, Cai S, Yang Q, Forrest ML. Multi-arm polymeric nanocarrier as a nitric oxide delivery platform for chemotherapy of head and neck squamous cell carcinoma. Biomaterials. 2012;33(11):3243-53.

72. Duong HT, Kamarudin ZM, Erlich RB, Li Y, Jones MW, Kavallaris M, et al. Intracellular nitric oxide delivery from stable NO-polymeric nanoparticle carriers. Chemical Communications. 2013;49(39):4190-2.

73. Kandoth N, Vittorino E, Sciortino MT, Parisi T, Colao I, Mazzaglia A, et al. A Cyclodextrin-Based Nanoassembly with Bimodal Photodynamic Action. Chemistry-A European Journal. 2012;18(6):1684-90.

74. Seabra AB, Durán N. Nitric oxide-releasing vehicles for biomedical applications. Journal of Materials Chemistry. 2010;20(9):1624-37.

75. Seabra AB, Marcato PD, de Paula LB, Durán N, editors. New strategy for controlled release of nitric oxide. Journal of Nano Research; 2012: Trans Tech Publ.

76. Yeo Y, Park K. Control of encapsulation efficiency and initial burst in polymeric microparticle systems. Archives of pharmacal research. 2004;27(1):1.

77. Edlund U, Albertsson A-C. Degradable polymer microspheres for controlled drug delivery. Degradable aliphatic polyesters: Springer; 2002. p. 67-112.

78. Jung T, Breitenbach A, Kissel T. Sulfobutylated poly (vinyl alcohol)-graft-poly (lactide-co-glycolide) $\mathrm{s}$ facilitate the preparation of small negatively charged biodegradable nanospheres. Journal of controlled release. 2000;67(2):157-69.

79. Seekell RP, Lock AT, Peng Y, Cole AR, Perry DA, Kheir JN, et al. Oxygen delivery using engineered microparticles. Proceedings of the National Academy of Sciences. 2016:201608438.

80. Jalil R, Nixon J. Microencapsulation using poly (dl-lactic acid) I: effect of preparative variables on the microcapsule characteristics and release kinetics. Journal of microencapsulation. 1990;7(2):229-44.

81. Jalil R, Nixon J. Biodegradable poly (lactic acid) and poly (lactide-co-glycolide) microcapsules: problems associated with preparative techniques and release properties. Journal of microencapsulation. 1990;7(3):297-325.

82. Sah E, Sah H. Recent trends in preparation of poly (lactide-co-glycolide) nanoparticles by mixing polymeric organic solution with antisolvent. Journal of Nanomaterials. 2015;16(1):61. 
83. Verma RK, Singh AK, Mohan M, Agrawal AK, Verma PR, Gupta A, et al. Inhalable microparticles containing nitric oxide donors: saying NO to intracellular Mycobacterium tuberculosis. Molecular pharmaceutics. 2012;9(11):3183-9.

84. Bodmeier R, Chen H. Preparation of Biodegradable Poly ( \pm ) lactide Microparticles Using a Spray-Drying Technique. Journal of Pharmacy and Pharmacology. 1988;40(11):754-7.

85. Bodmeier R, McGinity JW. Polylactic acid microspheres containing quinidine base and quinidine sulphate prepared by the solvent evaporation technique. II. Some process parameters influencing the preparation and properties of microspheres. Journal of microencapsulation. 1987;4(4):289-97.

86. Williams JR, Clifford AA, Al-Saidi SH. Supercritical fluids and their applications in biotechnology and related areas. Molecular biotechnology. 2002;22(3):263.

87. Wischke C, Schwendeman SP. Principles of encapsulating hydrophobic drugs in PLA/PLGA microparticles. International Journal of pharmaceutics. 2008;364(2):298-327.

88. Wang J, Schwendeman SP. Mechanisms of solvent evaporation encapsulation processes: prediction of solvent evaporation rate. Journal of pharmaceutical sciences. 1999;88(10):1090-9.

89. Bodmeier R, McGinity J. Solvent selection in the preparation of poly (DL-lactide) microspheres prepared by the solvent evaporation method. International journal of pharmaceutics. 1988;43(1-2):179-86.

90. Bodmeier R, McGinity JW. The preparation and evaluation of drug-containing poly (dl-lactide) microspheres formed by the solvent evaporation method. Pharmaceutical research. 1987;4(6):465-71.

91. Riccio DA, Schoenfisch MH. Nitric oxide release: Part I. Macromolecular scaffolds. Chemical Society Reviews. 2012;41(10):3731-41.

92. Yoo JW, Lee JS, Lee CH. Characterization of nitric oxide-rel easing microparticles for the mucosal delivery. Journal of Biomedical Materials Research Part A. 2010;92(4):1233-43.

93. Jeyanthi R, Thanoo B, Metha R, DeLuca P. Effect of solvent removal technique on the matrix characteristics of polylactide/glycolide microspheres for peptide delivery. Journal of Controlled Release. 1996;38(2-3):235-44.

94. Li M, Rouaud O, Poncelet D. Microencapsulation by solvent evaporation: State of the art for process engineering approaches. International Journal of pharmaceutics. 2008;363(1):26-39. 
95. Göpferich A. Mechanisms of polymer degradation and erosion. Biomaterials. 1996;17(2):103-14.

96. Freitas S, Merkle HP, Gander B. Microencapsulation by solvent extraction/evaporation: reviewing the state of the art of microsphere preparation process technology. Journal of controlled release. 2005;102(2):313-32.

97. Makadia HK, Siegel SJ. Poly lactic-co-glycolic acid (PLGA) as biodegradable controlled drug delivery carrier. Polymers. 2011;3(3):1377-97.

98. Fredenberg S, Wahlgren M, Reslow M, Axelsson A. The mechanisms of drug release in poly (lactic-co-glycolic acid)-based drug delivery systems - a review. International journal of pharmaceutics. 2011;415(1):34-52.

99. Huang X, Brazel CS. On the importance and mechanisms of burst release in matrixcontrolled drug delivery systems. Journal of controlled release. 2001;73(2):121-36.

100. Kamaly N, Yameen B, Wu J, Farokhzad OC. Degradable controlled-release polymers and polymeric nanoparticles: mechanisms of controlling drug release. Chem Rev. 2016;116(4):2602-63.

101. Ahmed EM. Hydrogel: Preparation, characterization, and applications: A review. Journal of advanced research. 2015;6(2):105-21.

102. Jagur-Grodzinski J. Polymeric gels and hydrogels for biomedical and pharmaceutical applications. Polymers for Advanced Technologies. 2010;21(1):27-47.

103. Kopecek J. Hydrogels: From soft contact lenses and implants to self-assembled nanomaterials. Journal of Polymer Science Part A: Polymer Chemistry. 2009;47(22):5929-46.

104. Slaughter BV, Khurshid SS, Fisher OZ, Khademhosseini A, Peppas NA. Hydrogels in regenerative medicine. Advanced materials. 2009;21(32-33):3307 -29.

105. Boateng JS, Matthews KH, Stevens HN, Eccleston GM. Wound healing dressings and drug delivery systems: a review. Journal of pharmaceutical sciences. 2008;97(8):2892-923.

106. Saltzman WM, Olbricht WL. Building drug delivery into tissue engineering design. Nature Reviews Drug Discovery. 2002;1(3):177-86.

107. Liu Y, Cai S, Shu XZ, Shelby J, Prestwich GD. Release of basic fibroblast growth factor from a crosslinked glycosaminoglycan hydrogel promotes wound healing. Wound repair and regeneration. 2007;15(2):245-51. 
108. Shin H, Jo S, Mikos AG. Biomimetic materials for tissue engineering. Biomaterials. 2003;24(24):4353-64.

109. Tabata Y. The importance of drug delivery systems in tissue engineering. Pharmaceutical science \& technology today. 2000;3(3):80-9.

110. Hoare TR, Kohane DS. Hydrogels in drug delivery: progress and challenges. Polymer. 2008;49(8):1993-2007.

111. Alkayyali LB, Abu-Diak OA, Andrews GP, Jones DS. Hydrogels as drug-delivery platforms: physicochemical barriers and solutions. Therapeutic delivery. 2012;3(6):775-86.

112. Bae JW, Go DH, Park KD, Lee SJ. Thermosensitive chitosan as an injectable carrier for local drug delivery. Macromolecular Research. 2006;14(4):461-5.

113. Takahashi T, Imai M, Suzuki I. High-potential molecular properties of chitosan and reaction conditions for removing p-quinone from the aqueous phase. Biochemical engineering journal. 2005;25(1):7-13.

114. Berger J, Reist M, Mayer JM, Felt O, Gurny R. Structure and interactions in chitosan hydrogels formed by complexation or aggregation for biomedical applications. European Journal of Pharmaceutics and Biopharmaceutics. 2004;57(1):35-52.

115. Huang S, Fu X. Naturally derived materials-based cell and drug delivery systems in skin regeneration. Journal of Controlled Release. 2010;142(2):149-59.

116. Ribeiro MP, Espiga A, Silva D, Baptista P, Henriques J, Ferreira C, et al. Development of a new chitosan hydrogel for wound dressing. Wound repair and regeneration. 2009;17(6):817-24.

117. Lin-Gibson WH, Kennedy S, Welsh E. Chitosan hydrogels: crosslink kinetics and gel properties. Polym Mater: Sci Eng. 2003;88:199.

118. Ostrowska-Czubenko J, Gierszewska-Drużyńska M. Effect of ionic crosslinking on the water state in hydrogel chitosan membranes. Carbohydrate Polymers. 2009;77(3):590-8.

119. Bhattarai N, Ramay HR, Gunn J, Matsen FA, Zhang M. PEG-grafted chitosan as an injectable thermosensitive hydrogel for sustained protein release. Journal of Controlled Release. 2005;103(3):609-24.

120. Goycoolea F, Argüelles-Monal W, Lizardi J, Peniche C, Heras A, Galed G, et al. Temperature and $\mathrm{pH}$-sensitive chitosan hydrogels: DSC, rheological and swelling evidence of a volume phase transition. Polymer Bulletin. 2007;58(1):225-34. 
121. Buckley CT, Thorpe SD, O'Brien FJ, Robinson AJ, Kelly DJ. The effect of concentration, thermal history and cell seeding density on the initial mechanical properties of agarose hydrogels. Journal of the mechanical behavior of biomedical materials. 2009;2(5):512-21.

122. Zamora-Mora V, Velasco D, Hernández R, Mijangos C, Kumacheva E. Chitosan/agarose hydrogels: Cooperative properties and microfluidic preparation. Carbohydrate polymers. 2014;111:348-55.

123. Sakai S, Hashimoto I, Kawakami K. Agarose-gelatin conjugate for adherent cellenclosing capsules. Biotechnology letters. 2007;29(5):731-5.

124. Gómez-Mascaraque LG, Méndez JA, Fernández-Gutiérrez M, Vázquez B, San Román J. Oxidized dextrins as alternative crosslinking agents for polysaccharides: application to hydrogels of agarose-chitosan. Acta biomaterialia. 2014;10(2):798811.

125. Zhang L-M, Wu C-X, Huang J-Y, Peng X-H, Chen P, Tang S-Q. Synthesis and characterization of a degradable composite agarose/HA hydrogel. Carbohydrate polymers. 2012;88(4):1445-52. 


\section{Particle synthesis and characterization}

\subsection{Introduction}

Nitric oxide (NO) is an important signaling agent present naturally in the body responsible for a variety of biological functions such as controlling hemostasis, regulating vascular tone, encouraging revascularization and fight infection (1). NO is also involved in physiological processes such as anti-inflammation (2) and wound healing $(3,4)$. The pharmaceutical application of $\mathrm{NO}$ is complex because of its short life, high reactivity and short diffusional distance (5). The therapeutic application of gaseous $\mathrm{NO}(\mathrm{gNO})$ is very complicated and expensive. Therefore, a stable NO storage material in the form of a wide range of NO donor molecules such as nitrites, S-nitroso (RSNO) compounds and diazeniumdiolates (NONOates) $(4,6,7)$ have been studied for NO delivery. Several studies have attempted to the use NO donors by blending or covalently attaching them to the biodegradable materials for controlled delivery of tunable levels of NO. Coneski et al. synthesized the NO releasing functionalized polyesters which delivered NO in the range of $0.0-2.28 \mu \mathrm{mol}(8)$.

NONOates and nitrosothiols (RSNOs) are the two most promising groups of small molecules NO donors recently studied for the delivery of NO (9). Ameer group developed another class of NO releasing biodegradable polyesters which are two different variations of diazeniumdiolated poly (diol-citrate) elastomers. In the first report Zhao et al. synthesized and characterized the first variation of elastomer film which released $\mathrm{NO}$ over a period of 3 days and has a degradation rate of $20 \%$ over 6 weeks (10). In the second report, Wang et al. employed the photo-crosslinking method to the elastomer system which augmented the NO release time to 7 days while reducing the degradation rate to between $26-32 \%$ over 6 months (11). 
Pulfer et al. prepared polyethylenimine microsphere derivatized with a NONOate moiety. These microspheres have a diameter of 10-50 $\mu \mathrm{m}$ with a release of $194 \mathrm{nmol} \mathrm{NO} / \mathrm{mg}$ and a half-life of $66 \mathrm{~h}$ under physiological conditions (12). In another study, a small molecule donor diazeniumdiolated proline (PROLI/NO) was encapsulated within biodegradable PLGA and polyethylene oxide-co-lactic acid (PELA) microspheres by Jeh et al. for delivery of NO (13). Zhang et al., produced fumed silica particles by first tethering alkylamines onto the surface of silica and then these amine groups were converted to corresponding $\mathrm{N}$-diazeniumdiolate groups via reaction with $\mathrm{NO}$ gas at high pressure which produced NO for few hours (14). Schoenfisch's group synthesized gold (15), silica nanoparticles (16) and polypropylenimine dendrimers (17) which were functionalized with NONOate moieties released NO for several hours. The same group also developed O2protected diazeniumdiolate-modified mesoporous silica nanoparticles which had the longest NO release time with a half-life of 3 weeks at $\mathrm{pH} 7.4$ (18). Diethylenetriamine (DETA) NONOate was successfully encapsulated into poly (lactic-co-glycolic acid) (PLGA) microparticles using solvent evaporation method by Yoo et al. The Deta nonoate loaded PLGA microparticles released $\sim 0.3 \mu \mathrm{mol} \mathrm{NO} / \mathrm{mg}$ polymer in a controlled manner for just over 6 h (19). Similarly, Nurhasni et al. developed NO releasing poly(lactic-coglycolic acid)-polyethylenimine (PEI)/ diazeniumdiolate nanoparticles (NO/PPNPs) with an average size of $162 \pm 19 \mathrm{~nm}$ for antibacterial and wound healing activity (20). The PEI was selected as the NO donor polymer. The synthesized PEI/diazeniumdiolate (PEI/NONOate) was incorporated in to PLGA nanoparticles resulting in NO release capacity of $1.4 \mu \mathrm{mol} \mathrm{NO} / \mathrm{mg}$ of polymer with a release lifetime of over 6 days. The main drawback of these particles in general is possibility of formation of carcinogenic $\mathrm{N}$ nitrosamines as a side product during the production of $N$-diazeniumdiolates using polyamine compounds (21).

Another NO donor employed for storage and delivery of NO is S nitrosothiol (RSNO) which are endogenously produced in vivo and are non-toxic. Several mechanisms such as heat, light, exposure to copper and ascorbic acid triggers their decomposition and release NO (22-24). 
The different RSNO decomposition mechanisms are useful for their bio applications as in vivo body conditions can stimulate the NO release from RSNOs. Researchers have utilized S-nitrosothiols as NO donor within polymer systems. Gierke et al. synthesized a silicon rubber material in which SNAP was covalently linked to polydimethylsiloxane (SNAPPDMS). This material released NO via light trigger (25). Brisbois et al. investigated and found two low water uptake biomedical grade polymers Elast-eon E2As and CarboSil have potential to control the release of NO from the SNAP. When doped within these polymers, SNAP is exceptionally stable within them and can release NO up to 3 weeks at physiological conditions $(26,27)$. In another study, Wo et al. demonstrated that long term NO release can be achieved from carbosil-SNAP because of slow crystal dissolution process as SNAP concentration in carbosil when exceeds its solubility makes the SNAP present in carbosil in crystalline form. The hydrophobicity of the carbosil polymer and stabilization of crystalline SNAP by intermolecular hydrogen bonds limits the water diffusion only to the polymer surface which keeps this composite stable during storage for at least 8 months $(28,29)$.

Frost et al. anchored RSNOs to fumed silica particles with a NO loading of 21-138 $\mathrm{nmol} / \mathrm{mg}$ and blended into polyurethane and silicone rubber matrixes to create films that release $\mathrm{NO}$ at controlled rates. The formed films embedded with particles were capable of NO release when exposed to copper(II) ions or ascorbic acid or upon shining light (30). A RSNO modified polyamidoamine (PAMAM) dendrimer was developed by Stasko et al., which can store up to $2 \mu \mathrm{mol} \mathrm{NO}$ per $\mathrm{mg}$ of dendrimer. They can release NO for $10 \mathrm{~min}$ to several hours via copper(II) and light exposure (31). These types of particles have an issue as the NO donors in these systems are immobilized on the surface exposed to the matrix environment. The presence of even tiny amounts of copper or ascorbate ions can induce burst release which is a problematic effect. Lautner et al. encapsulated SNAP into PLGA (RG 503H and RG 504) microspheres using solid-oil-in water (S/O/W) emulsion method. The microspheres were $20-125 \mu \mathrm{m}$ in size and exhibited controlled localized NO release from PLGA RG 504 in the presence of copper(II) and ascorbate (32). 
The aim of this study was to develop a microparticle based NO delivery system that could provide physiologically relevant levels of NO for proper wound healing without yielding any toxic by-product. The NO donor used in this study i.e. S-nitroso-N-acetyl-Dpenicillamine (SNAP) breaks down into N-acetyl-L-penicillamine (NAC) after the loss of NO which is non-toxic at low levels (33). Even after the loss of acetate group from the NAC, the product penicillamine itself is FDA approved for reversing heavy metal ion poisoning (34).Herein, PLLA microparticles encapsulated with S-nitroso-N-acetyl-Dpenicillamine (SNAP) have been developed for efficiently delivery of NO. PLLA microparticles were prepared using double emulsion method at two different stirring speeds. The size, morphology, and encapsulation efficiency of these microparticles were evaluated. The controlled NO release profile of SNAP loaded microparticles was assessed using light activated release. The total amount of NO released via combination of light, copper (II) and ascorbate ion was also explored.

\subsection{Material and method}

\subsubsection{Materials}

Microparticles were prepared using biodegradable poly (l-lactide) acid (PLLA) granules purchased from NatureWorks LLC (Blair, NE, USA). Emulsifying agent poly vinyl alcohol (PVA) with molecular weight of 9,000-10,000 80\% hydrolyzed was purchased from Aldrich (St. Louis, MO, USA). Chelator for transition metal ion Chelex ${ }^{\circledR} 100$ resin (100200 mesh, sodium form) and Cyclam (1, 4, 8, 11-tetraazacyclotetradecane) were obtained from Bio-Rad (Hercules, CA, USA) and Acros Organics (Geel, Belgium) respectively. L$(+)$-Ascorbic Acid used for experiments was purchased from J.T Baker $(>=99.5 \%)$ (Center Valley, PA, USA). Copper chloride $\left(\mathrm{CuCl}_{2}\right)$ anhydrous was purchased from Alfa Aesar (Haverhill, MA). DMSO and Dichloromethane were obtained from Sigma-Aldrich (St. Louis, MO, USA). For stirring emulsion, air stirrer (Model no. A) with a speed ranging from rpm 200 - 10,000 and air input pressure ranging from 30 -100 psi was purchased from Arrow Engineering Co. (Pennsylvania, N.J., USA). 


\subsubsection{SNAP synthesis}

$\mathrm{N}$-acetyl-D-penicillamine, and sodium nitrite (99.999\% trace metals basis) were obtained from Sigma-Aldrich (St. Louis, MO, USA). For the synthesis of S-nitroso-Nacetylpenicillamine (SNAP) crystals, $200 \mathrm{mg}$ of $\mathrm{N}$-acetylpenicillamine (NAP) was dissolved in $5 \mathrm{ml}$ methanol and sonicated for 5 mins. The solution was acidified by slowly adding two milliliters of $1 \mathrm{M} \mathrm{HCl}$ and $100 \mu \mathrm{l}$ of concentrated $\mathrm{H}_{2} \mathrm{SO}_{4}$. Then $144.9 \mathrm{mg} \mathrm{NaNO} 2$ were added to the acidified NAP solution and vortex mixed till all the sodium nitrite was dissolved avoiding light. A dark green/red color in the SNAP solution gradually developed and the solution was ice cooled for $45 \mathrm{~min}$. After cooling, the methanol in the SNAP solution was evaporated by rotary evaporation and SNAP crystals were collected using vacuum filtration. The crystals were washed thrice by $\mathrm{diH}_{2} \mathrm{O}$ repeatedly and air-dried. The SNAP crystals were kept in refrigerator $4^{\circ} \mathrm{C}$ in dark until further use.

\subsubsection{S-nitroso-N-acetylpenicillamine (SNAP) loaded poly (l-lactic) - polyvinyl alcohol microparticles}

\subsubsection{Preparation of SNAP loaded microparticles}

Biodegradable SNAP loaded PLLA microparticles were prepared using water-in-oil-water $(\mathrm{W} / \mathrm{O} / \mathrm{W})$ solvent evaporation method. This method was chosen over much simpler waterin-oil solvent evaporation because S-nitroso-N-acetylpenicillamine (SNAP) is insoluble in dichloromethane and partially soluble in water which can lead to drug loss during preparation method. S-nitroso-N-acetylpenicillamine (SNAP) was dissolved in dimethyl sulfoxide (DMSO) as it is completely soluble in DMSO.

The schematic representation of the snap-loaded microparticle production is shown in Fig 2.1. First, 20\% (w/w) PLLA polymer solution was prepared. For this, $200 \mathrm{mg}$ of poly (1lactic acid) (PLLA) was dissolved in $1 \mathrm{ml}$ of dichloromethane (DCM) in a $20 \mathrm{ml}$ disposable scintillation vials. $10 \mathrm{mg}$ cyclam $(1,4,8,11$-tetraazacyclotetradecane) were added to the polymer solution to bind any copper ion present in the solution. Then, $20 \mathrm{mg}$ of SNAP were taken in an Eppendorf tube and dissolved in 100 $\mu$ l dimethyl sulfoxide (DMSO) and vortex mixed. The SNAP solution was poured to the polymer solution and vortexed gently 
avoiding light. The polymer-SNAP solution was sonicated for $2 \mathrm{~min}$ in a bath sonicator. This forms the primary (water in oil) emulsion which is green in color. In a beaker, 1\% (w/w) poly (vinyl) alcohol (PVA) was prepared as an emulsifier. For this, in a beaker 500 mg of PVA were dissolved in $50 \mathrm{ml}$ chelex containing $0.01 \mathrm{M} \mathrm{HCl}$ using a magnetic stirrer. Chelex is used as a chelator to bind any copper ion in the $\mathrm{diH}_{2} \mathrm{O}$. Chelex when added to $\mathrm{diH}_{2} \mathrm{O}$ raise the $\mathrm{pH}$ and make it basic. $\mathrm{HCl}$ was added to the PVA emulsifying solution to prevent decomposition of SNAP at a basic $\mathrm{pH}$ as SNAP is stable at acidic $\mathrm{pH}$ levels. The primary solution was then introduced dropwise to aqueous 1\% PVA solution containing $0.01 \mathrm{M} \mathrm{HCl}$ to form water in oil in water $(\mathrm{W} / \mathrm{O} / \mathrm{W})$ emulsion. The final emulsion was stirred using air stirrer at two different speeds $500 \mathrm{rpm}$ and $2500 \mathrm{rpm}$ at $25^{\circ} \mathrm{C}$ for $3 \mathrm{~h}$ in a fume hood to remove organic solvent. While stirring, the final solution was kept in an ice bath to prevent thermal decomposition of SNAP. The microparticles were collected by centrifuge at 2000 RPM and then recovered by washing three times with chelex treated $\mathrm{diH}_{2} \mathrm{O}$. The microparticles were air dried for $24-48 \mathrm{~h}$ and stored at $-20^{\circ} \mathrm{C}$ until further usage.

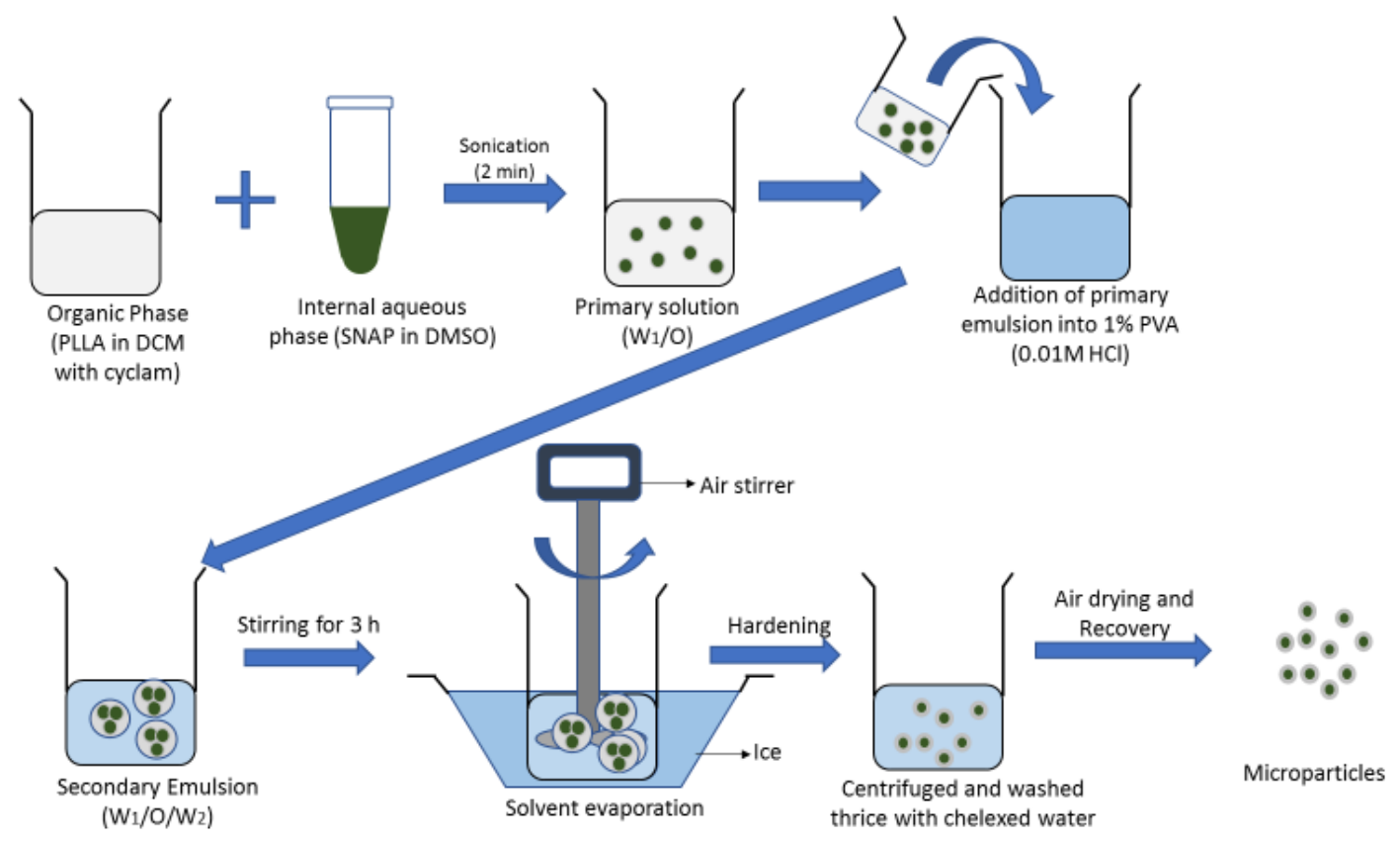

Figure 2.1 Preparation of the microparticles using double emulsion solvent evaporation method. Produced microparticles were collected, air dried and stored in dark at $-20{ }^{\circ} \mathrm{C}$. 


\subsubsection{Yield of the prepared microparticles}

Yield of the microparticles prepared by double emulsion was calculated to evaluate the effectiveness of the method. It is calculated using equation:

Yield $=\underline{\mathrm{W} \text { (microparticles) }}$

W (total)

$\mathrm{W}$ (microparticle) is the weight of the produced microparticles

$\mathrm{W}$ (total) is the total weight of the polymers and the drug used to prepare microparticles

\subsubsection{Characterization of the SNAP loaded microparticles}

The morphology, size and size distribution of the SNAP loaded and blank microparticles both 500 and $2500 \mathrm{rpm}$ were investigated by using scanning electron microscope (SEM) with a Hitachi S-4700 field emission-scanning electron microscopy (FE-SEM). Samples were prepared by mounting a small amount of microparticles to the two-sided carbon tape on the top of the metallic sample holder. Then the samples were sputter coated with $5 \mathrm{~nm}$ of platinum-palladium (Pt/Pd) alloy with a current of $20 \mathrm{~mA}$ using Cressington $208 \mathrm{HR}$ with a MTM-20 film thickness monitor. Size distribution was determined by measuring the diameters of microparticle population from SEM image using ImageJ software.

\subsubsection{UV-Vis analysis of SNAP loaded microparticles}

UV-Vis spectroscopy was used to determine the SNAP loading in the microparticles. To determine the SNAP concentration in each batch of microparticles, 20mg of the microparticles were dissolved in $2 \mathrm{ml}$ of $3: 2$ dichloromethane to methanol. The concentration of SNAP was measured by measuring absorbance at $340 \mathrm{~nm}$ using UV-Vis

(Perkin Elmer Lambda 35 spectrometer (Waltham, MA). The absorbance coefficient of SNAP was calculated by taking absorbance of different concentration of pure SNAP in dichloromethane: methanol solution.

Encapsulation Efficiency $=($ Actual Loading $* 100) /$ Theoretical Loading $)$ 
Where, actual loading $=($ weight of SNAP in analyzed microparticles*100)/weight of microparticles used in UV-Vis analyses

Theoretical loading $=($ weight of SNAP added in microparticles*100)/weight of polymer and SNAP added during the preparation

\subsubsection{In vitro nitric oxide release study of SNAP loaded microparticles using Nitric Oxide Analyzer}

The NO release measurements were quantified using ozone based chemiluminescence detection with a Sievers 280i Nitric Oxide Analyzer (NOA) (GE Instruments, Boulder, CO, USA) using $200 \mathrm{ml} / \mathrm{min}$ flow rate, with both house nitrogen as the sweep gas. The instrument was calibrated with Zero NO gas and 45 PPM standard calibration NO (Air Liquid Healthcare America Corp. Plumsteadville, PA). Nitrogen was used as a sweep gas for carrying NO into NOA.

\subsubsection{Controlled NO release analysis}

For controlled light-initiated NO release measurements, 5mg SNAP loaded microparticles were placed in an amber vial and illuminated with different light intensity from a $470 \mathrm{~nm}$ LED (VAOL-5GSBY4, Mouser electric, Mansfield, TX) using variable voltage (3V, 4.5V, $6 \mathrm{~V}, 7.5 \mathrm{~V}, 9 \mathrm{~V}, 12 \mathrm{~V})$. The $\mathrm{NO}$ release was measured for $2 \mathrm{~min}$ at each voltage first increasing and then decreasing.

\subsubsection{Total NO release analysis}

For total NO release, $5 \mathrm{mg}$ of microparticles were placed in amber vial with a magnetic stir bar attached to the LED source. $0.5 \mathrm{ml}$ solution of $50 \mu \mathrm{M} \mathrm{CuCl}_{2}$ and $100 \mathrm{mM}$ ascorbic acid was added to the microparticles after a plateau was reached in the detected NO concentration, which is usually $15 \mathrm{~min}$. Along with the $\mathrm{CuCl}_{2}$ and ascorbic acid solution, microparticles were illuminated with same LED light at $4 \mathrm{~V}$. 
Both NO release experiments were performed at room temperature. The NO levels in ppb unit were converted to moles $\mathrm{sec}^{-1} \mathrm{mg}^{-1}$ unit using the NOA instrument constant that was determined by quantitative reduction of a known quantity of nitrite. Integration of NO release curves for given period was done to calculate the amount of NO released in both experiments.

\subsubsection{Statistical analysis}

The statistical analysis of the data was done using MS-excel 2016. All the data were shown as the means $( \pm \mathrm{SD})$. Two-tailed homoscedastic student's t-test was used to statistically analyze the data. The $p$ value $(<0.05)$ was considered significant. The graphs were made using the GraphPad Prism version 7.00 for Windows, GraphPad Software (La Jolla, California, USA) (www.graphpad.com).

\subsection{Results}

\subsubsection{SNAP loaded PLLA microparticles}

SNAP loaded microparticles were successfully prepared using $\mathrm{w} / \mathrm{o} / \mathrm{w}$ double emulsion method with air driven stirrer by air engineering co. (Pennsylvania, N.J.) at varying stirrer speed parameter. In this study PLLA polymer was used for encapsulation of SNAP which is a slow degrading polymer. The double emulsion system was used because the PLLA polymer dissolves in methylene chloride but the NO donor SNAP does not. In this study, DMSO was used to encapsulate as an inner aqueous solvent for dissolving SNAP. In this study, microparticles prepared at stirring rate of 500RPM and 2500RPM are denoted as microparticles $(500)_{\text {and }}$ microparticles $(2500)$.

\subsubsection{Yield of the microparticles}

Yield of the microparticle was calculated to evaluate the effectiveness of the preparation method. Yield of microparticles produced at both the stirring speeds were calculated using equation number 1 . Results and standard deviations are reported in the table 1. 
Table 1 Yield of produced microparticles

\begin{tabular}{l|l}
\hline \multicolumn{1}{c|}{ Composition } & \multicolumn{1}{c}{ yield (\%) \pm s.d. } \\
\hline SNAP-PLLA Microparticles (500) & $61.78 \pm 5.4$ \\
SNAP-PLLA Microparticles (2500) & $54.4 \pm 4$ \\
\hline
\end{tabular}

There is no significant difference $(\mathrm{p}=0.133)$ in the yield of microparticles produced at different speeds.

\subsubsection{Characterization of microparticles}

\subsubsection{Size and morphology of SNAP loaded microparticles}

Microparticles produced looks like white powder when visually observed. For more precise observations scanning electron microscope (SEM) was employed. Observed microparticles showed cluster formation and smaller particles adhered to larger particles. On visual observation, smaller microparticles ( $<150 \mu \mathrm{m}$ approx.) appear to be spherical without any irregularities. On the other hand, larger microparticles ( $>150 \mu \mathrm{m}$ approx.) appear to have irregular appearance and show either fully collapsed structured or pits on the surface of the microparticles. The SNAP loaded microparticles have smooth surface topography with visible pores which can be observed in fig 3.1 (d), (f), (h) and fig 3.2 (l), (n), (p). The blank microparticles, consisting of only PLLA however, have a rough surface with visible pores fig 3.1 (b) and fig 3.2 (j). Irregularities can be seen on the surface of the larger microparticles denoted by asterisk $(*)$.

Size distribution of SNAP loaded PLLA microparticles was measured using image J analysis tool and at least 150 microparticles were measured for each picture. The microparticles prepared at different speed (500 RPM and 2500 RPM) showed significant difference $(\mathrm{p}=0.02)$ in size. The average size measured of the SNAP loaded microparticles prepared at $500 \mathrm{RPM}$ stirring rate was $256.7 \pm 11.7 \mu \mathrm{m}$ while microparticles at $2500 \mathrm{RPM}$ were $207.4 \pm 19.7 \mu \mathrm{m}$. The blank microparticles, surprisingly, showed a smaller size 
compared to the SNAP loaded microparticles for both stirring speeds. Most of the microparticles were in the range of $\sim 100-300 \mu \mathrm{M}$ with some below $100 \mu \mathrm{m}$ and some above $300 \mu \mathrm{m}$.

It has to be emphasized that size measuring method here may not be accurate. It is possible to use tools that can be used for accurate diameter measurements from SEM images and the from size distribution of the sample. For these accurate measurements, the sample has to be prepared as a monolayer of the separated microparticles. Microparticles may be in different focus planes due to three-dimensional cluster formation which can affect the diameter measurements as some microparticles may be either near or farther behind microscope. For this analysis, oval and elliptical tool of the ImageJ software was used to measure every single microparticle by hand. Scale was set to match the scale bar of each SEM image. This method of measuring diameter of microparticles along with the image quality may cause significant inaccuracy to the result. Therefore, microparticle size distribution has to be considered approximate. 


\subsubsection{SEM images}

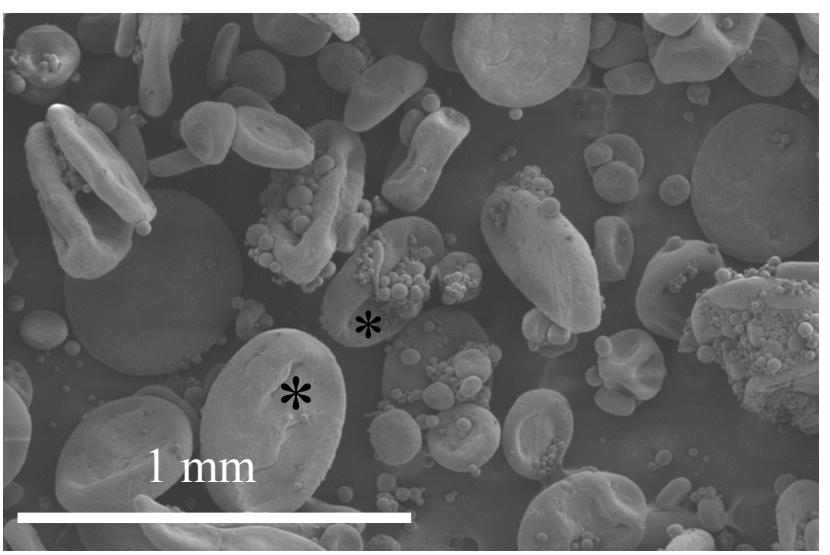

(a)

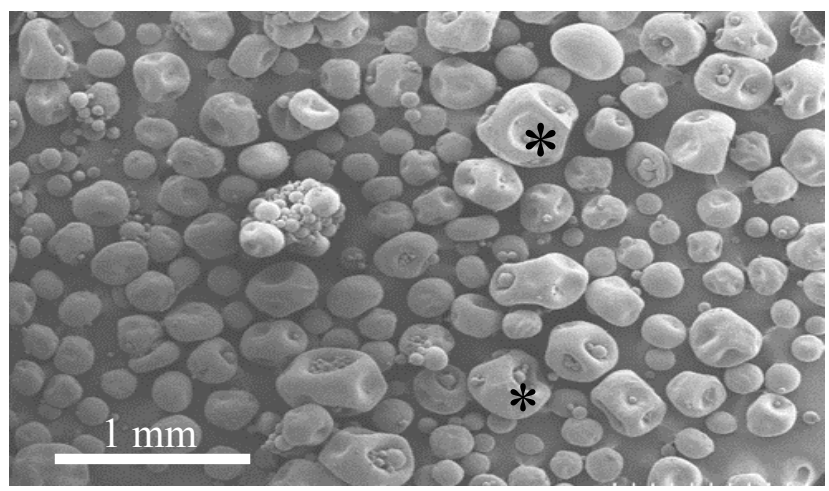

(c)

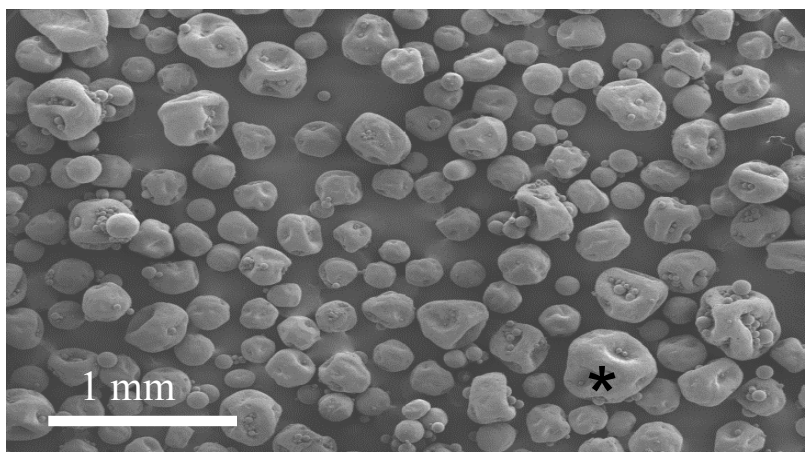

(e)

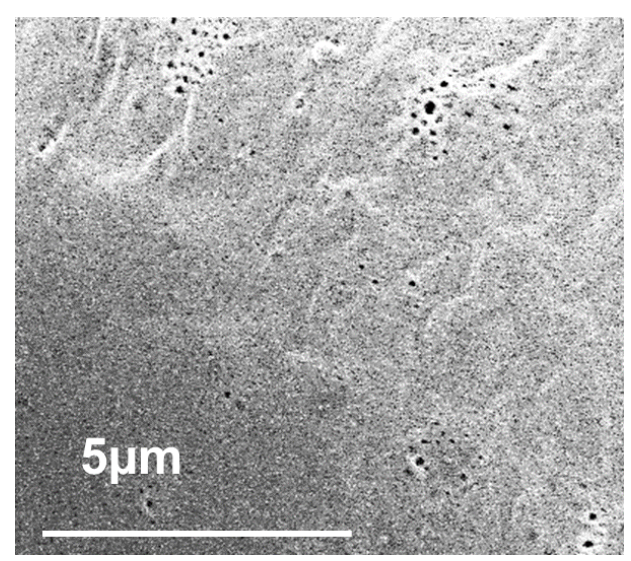

(b)

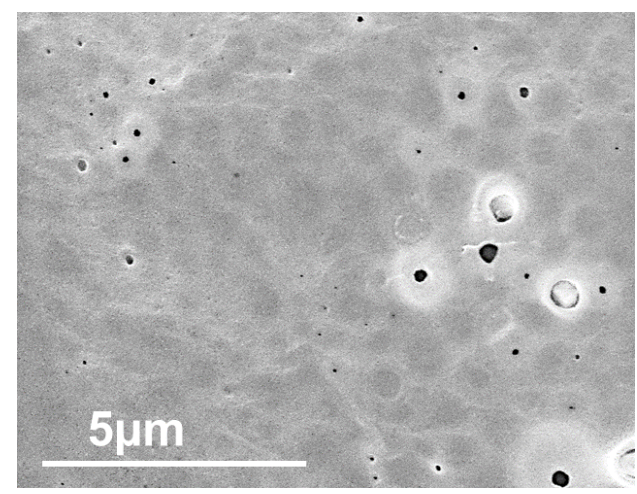

(d)

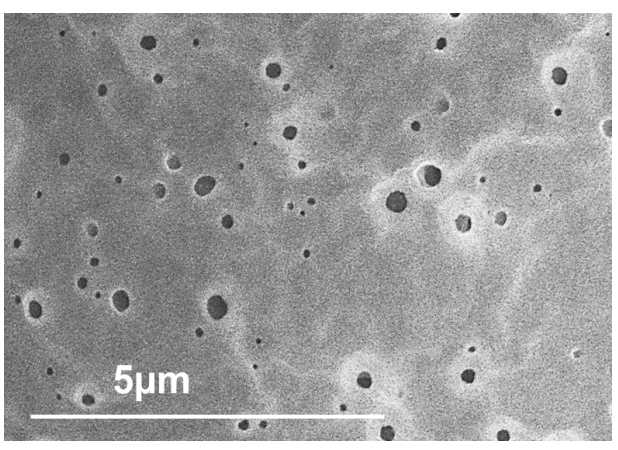

(f) 


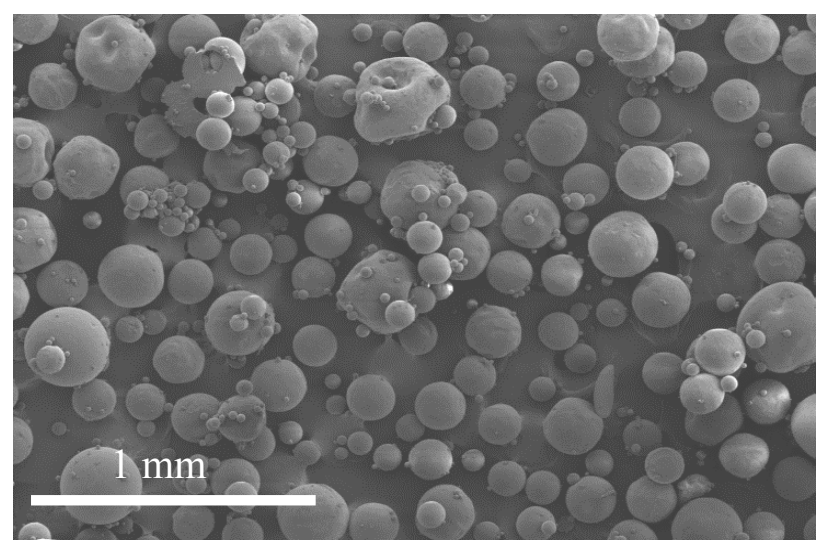

(g)

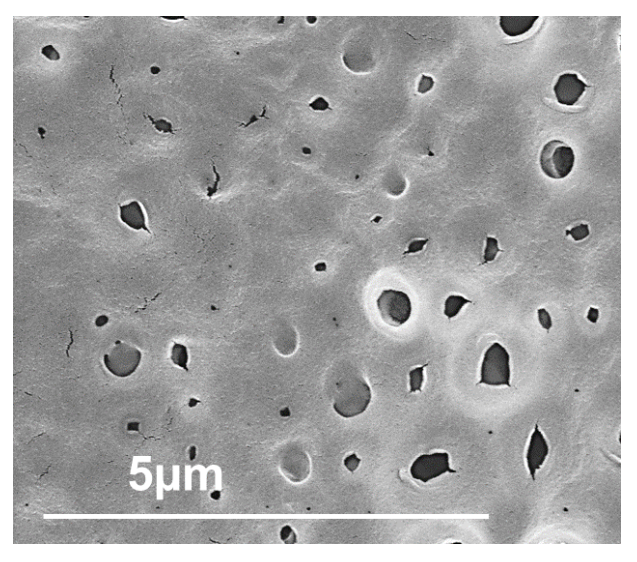

(h)

Figure 2.2 SEM images of microparticles made at 500 RPM (a), (c), (e), (g) shows the blank microparticle and SNAP loaded microparticle I, II, III respectively at magnification $1.00 \mathrm{~mm}$. (b), (d), (f), (h) surface topography of blank microparticle and SNAP loaded microparticle I, II, III respectively. 


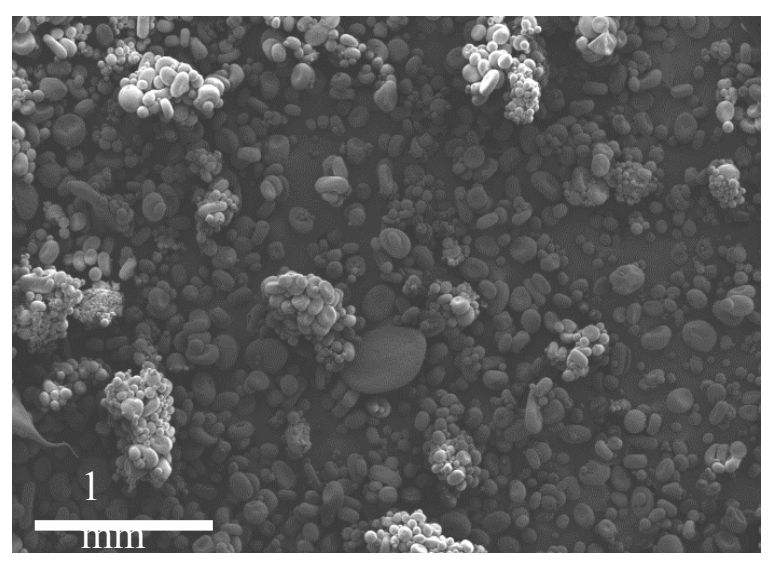

(i)

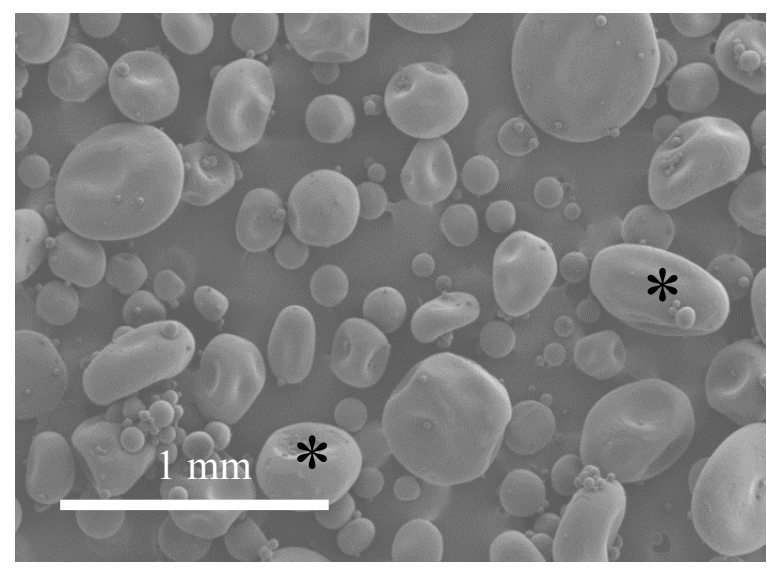

(k)

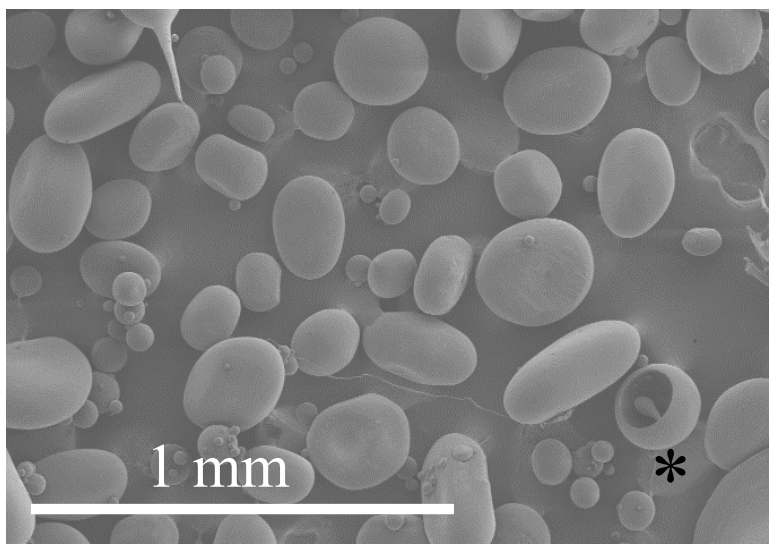

(m)

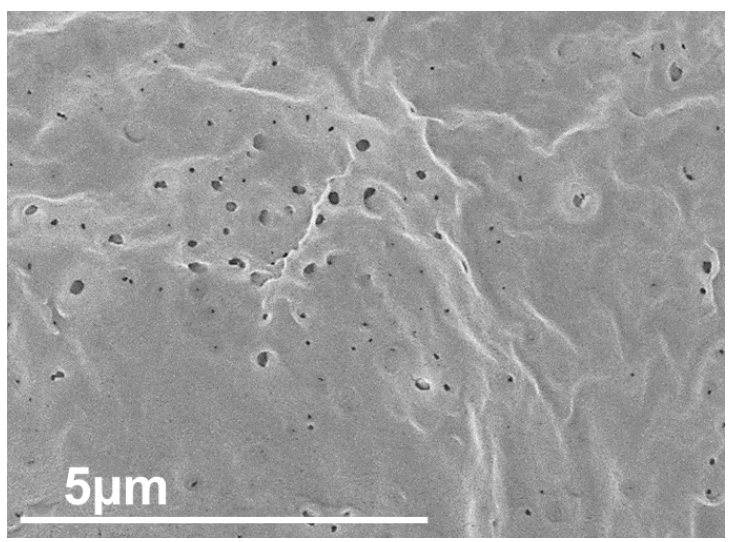

(j)

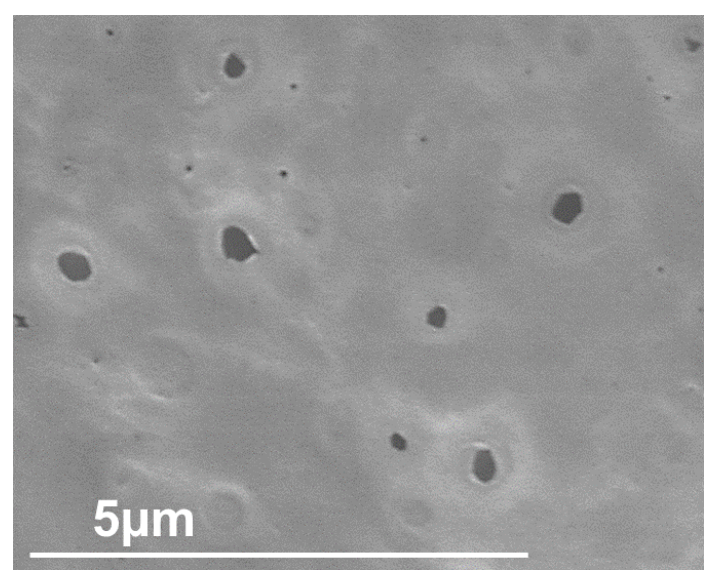

(I)

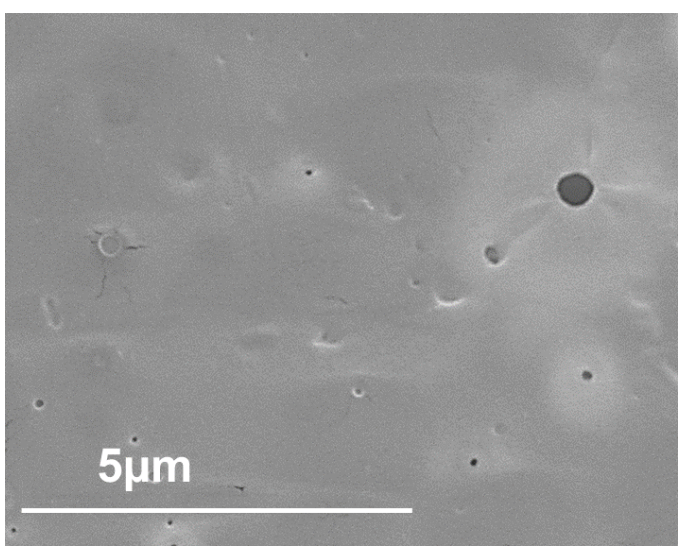

(n) 


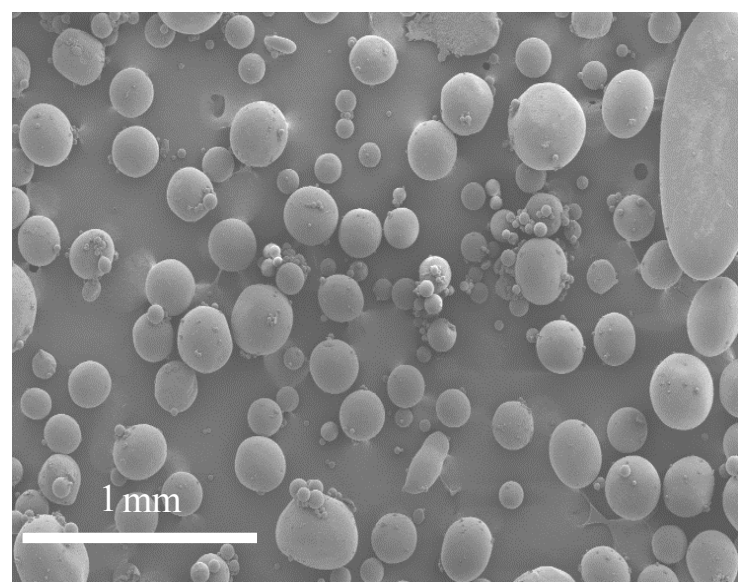

(0)

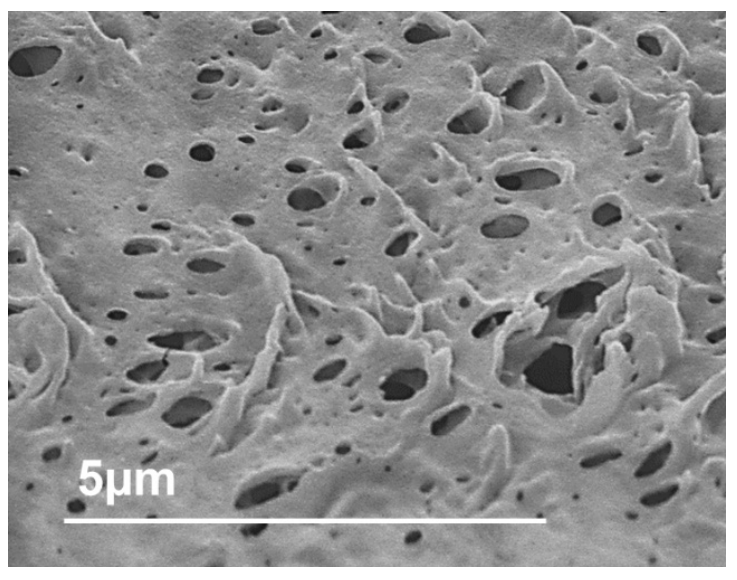

(p)

Figure 2.3 SEM images of microparticles made at 2500 RPM (i), (k), (m), (o) shows the blank microparticle and SNAP loaded microparticle I, II, III respectively at magnification $1.00 \mathrm{~mm} .(\mathrm{j}),(1),(\mathrm{n}),(\mathrm{p})$ surface topography of blank microparticle and SNAP loaded microparticle I, II, III respectively.

\subsubsection{Encapsulation efficiency of the SNAP loaded microparticles}

\subsubsection{UV-Vis analysis of SNAP loaded microparticles}

The SNAP loading of the PLLA microparticles were determined by UV/VIS absorption after dissolving the microparticles in DCM: methanol (3:2). The molar absorptivity of SNAP in DCM: methanol was determined by making standard curve of SNAP (Fig. 3.3) which was calculated by taking absorbance of dissolved SNAP in DCM: methanol at different concentrations. 


\section{Standard curve of SNAP in DCM:Methanol (3:2)}

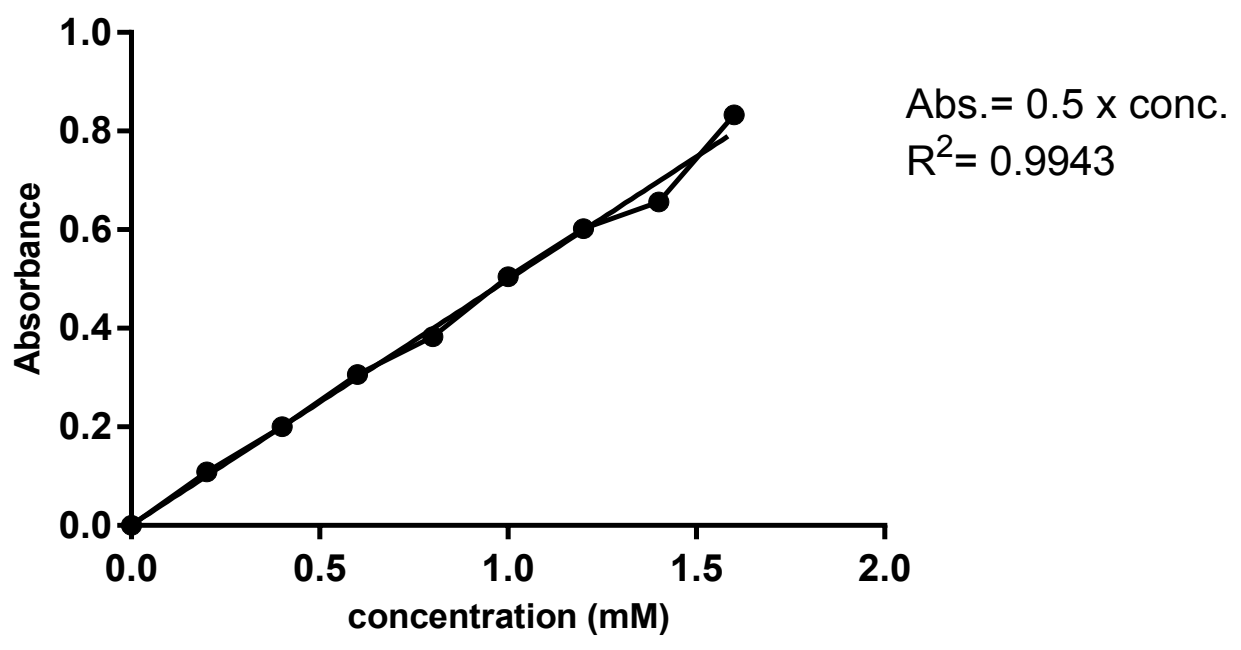

Figure 2.4 Standard curve of SNAP in DCM: Methanol (3:2)

The UV-Vis analysis of the SNAP loaded microparticles(500) and SNAP loaded microparticles(2500) are shown below in Fig. 3.4 and Fig. 3.5. The molar absorptivity of SNAP in DCM: methanol at $340 \mathrm{~nm}$ was determined as $\varepsilon S N A P=500 \mathrm{M}^{-1} \mathrm{~cm}^{1}$. The characteristic absorbance of SNAP at 340 correlated to the $\pi \rightarrow \pi^{*}$ electronic transitions of the S-NO functional group. 


\section{Microparticles (500 RPM)}

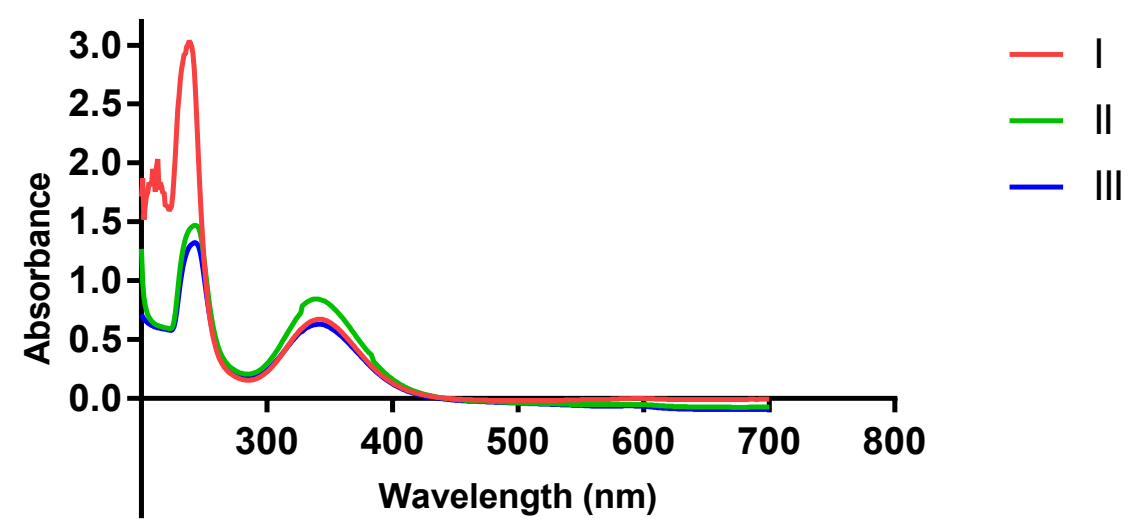

Figure 2.5 UV-vis analysis of SNAP loaded microparticles $(500)(\mathrm{n}=3)$

\section{Microparticles (2500 RPM)}

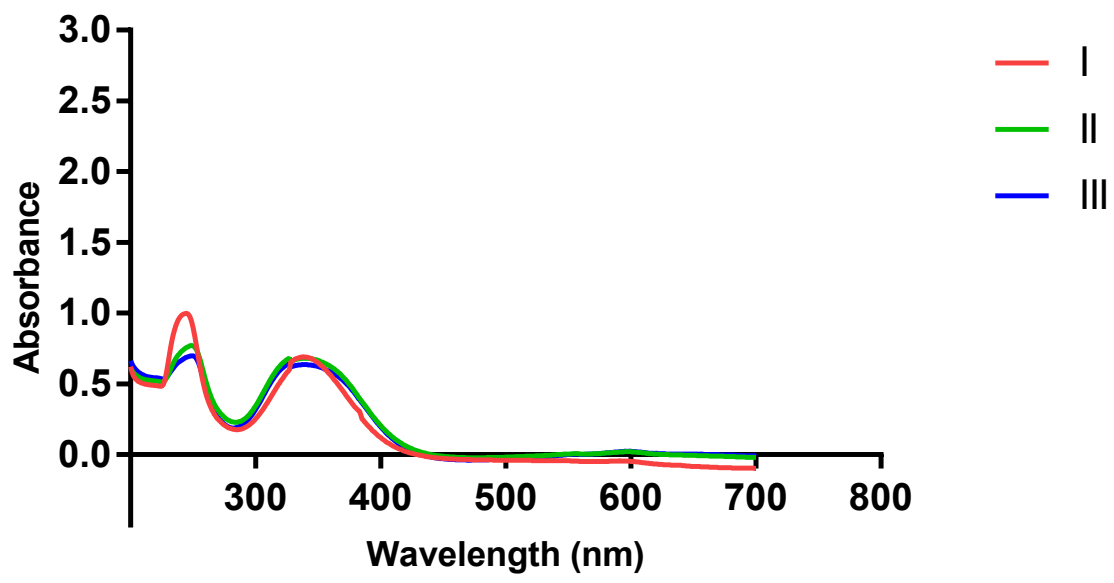

Figure 2.6 UV-vis analysis of SNAP loaded microparticles $(2500)(\mathrm{n}=3)$

The double emulsion method (W/O/W) yielded PLLA microparticles encapsulating SNAP an encapsulation efficiency of $\sim 35 \%$ for microparticle $(500)$ and $\sim 33 \%$ for microparticles(2500) (see Table 2). There was no significant difference $(p=0.54)$ in the encapsulation efficiency between the PLLA microparticle(500) and PLLA microparticles(2500). The amount of encapsulated SNAP was approximately 0.13-0.17 $\mu \mathrm{mol}$ per mg microsphere. 
Table 2 Composition (W/O/W), stirring speed, loadings, and particle size distribution and encapsulation efficiency

\begin{tabular}{|l|l|l|l|l|l|}
\hline Composition & $\begin{array}{l}\text { Stirring } \\
\text { speed } \\
(\mathrm{RPM})\end{array}$ & $\begin{array}{l}\text { SNAP } \\
\text { amount } \\
(\mathrm{mg})\end{array}$ & $\begin{array}{l}\text { particle size } \\
(\mu \mathrm{m})\end{array}$ & $\begin{array}{l}\text { Loading } \\
(\mathrm{w} / \mathrm{w}) \%\end{array}$ & $\begin{array}{l}\text { Encapsulation } \\
\text { efficiency (\%) }\end{array}$ \\
\hline $\begin{array}{l}\text { Blank-PLLA } \\
\text { microparticle }\end{array}$ & 500 & 0 & $190 \pm 21.3^{*}$ & n.a & n.a \\
\hline $\begin{array}{l}\text { SNAP-PLLA } \\
\text { Microparticle }\end{array}$ & 500 & 20 & $256 \pm 11.7^{* *}$ & $3.2 \pm 0.51$ & $35.2 \pm 5.6$ \\
\hline $\begin{array}{l}\text { Blank-PLLA } \\
\text { microparticle }\end{array}$ & 2500 & 0 & $130^{*} \pm 69.7^{*}$ & n.a & n.a \\
\hline $\begin{array}{l}\text { SNAP-PLLA } \\
\text { Microparticle }\end{array}$ & 2500 & 20 & $207 \pm 19.7^{* *}$ & $3.0 \pm 0.13$ & $33.0 \pm 1.4$ \\
\hline
\end{tabular}

* Standard deviation of different microparticle size in a single batch of microparticles $(n=1)$

** Standard deviation of different microparticle size in three different batches of microparticles $(n=3)$

\subsubsection{In vitro NO release profile study of SNAP loaded microparticles}

\subsubsection{Controlled NO release study via Light}

For in vitro monitoring of NO release from the microparticles, an amber vial was employed to contain the microparticles to avoid light induced NO photo-release. NO donor SNAP is sensitive to light which can release NO in a highly controllable manner. When SNAP is encapsulated in PLLA microparticles, photoinitiation of SNAP-PLLA microparticles was done to evaluate controlled NO release from them. LED light was applied to the microparticles to trigger the NO release. The SNAP loaded PLLA microparticles in dry powdered form when irradiated with light with a varying intensity. The microparticles showed a linear response with varying light intensities. Fig 6. (A), (B) demonstrates that the NO release was controlled by altering the drive voltage applied to the LED, thus changing the intensity of light produced by LED. 
In the absence of light, virtually no nitric oxide was released (trace a). However, as the light incident on SNAP microparticles is turned on and the light intensity is increased by stepping through $0,3,4.5,6,7.5,9$ and $12 \mathrm{~V}$ of applied potential (traces a, b, c, d, e, f and g), the NO release increases. The produced NO was purged out from the vial with a continuous $\mathrm{N}_{2}$ stream which is detected by the gold standard for NO release measurements that is chemiluminescence method. The voltage was kept $0 \mathrm{~V}$ usually for 15 mins to measure the NO concentration of the purged gas until a plateau reached in NO detection. The light intensity was kept constant for exact 2 mins at every voltage point and then decreased in the same manner. The SNAP loaded microparticles show an immense potential as a controlled NO-releasing material. This shows that light can be used as an external stimulus to trigger NO release which allows precise control of turning on and off mechanism for increasing and decreasing NO flux. This can provide an accurate amount of NO levels required to achieve specific physiological responses such as wound healing.

NO release from both SNAP-PLLA microparticle(500) and SNAP-PLLA microparticles(2500) was studied using this light method. 


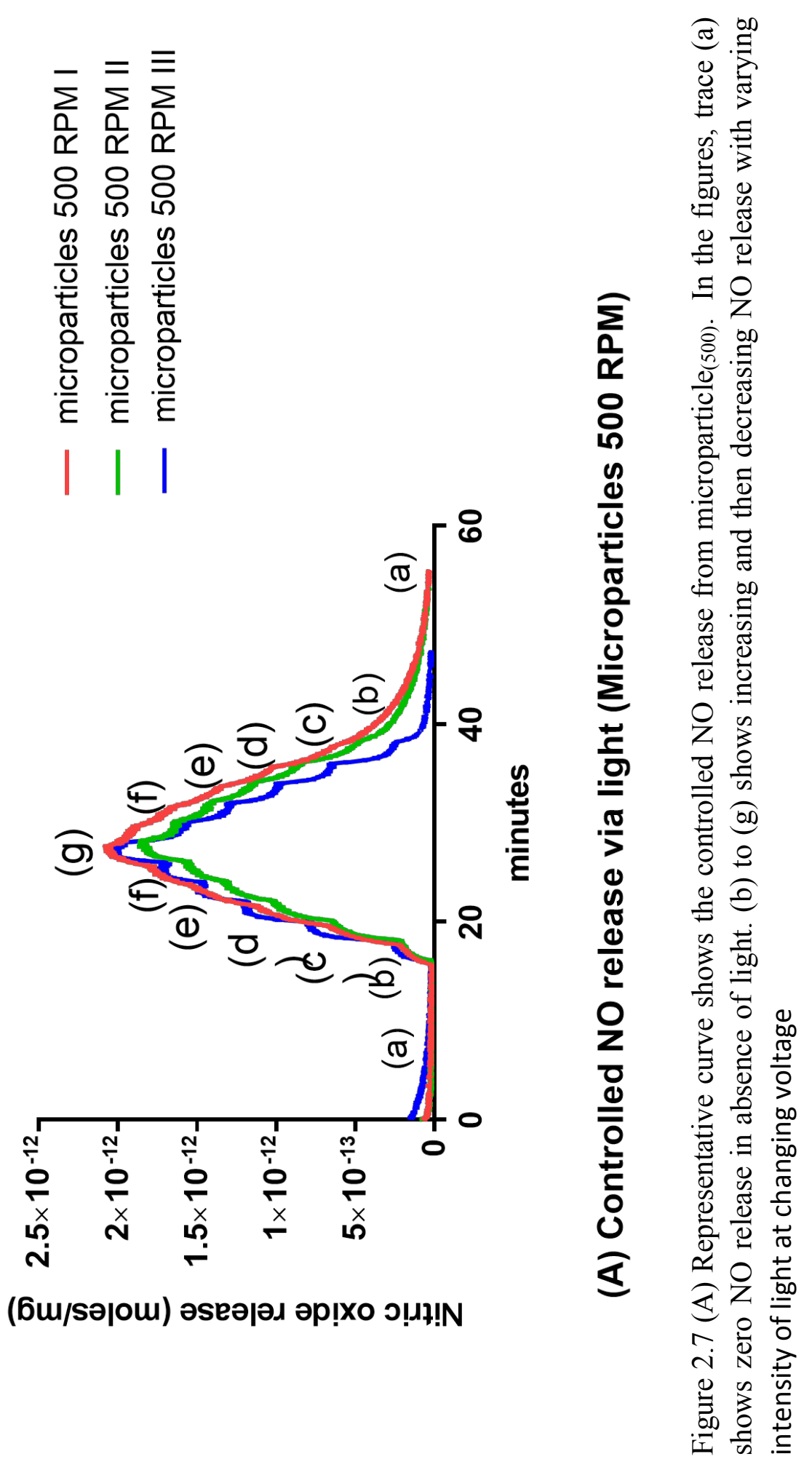




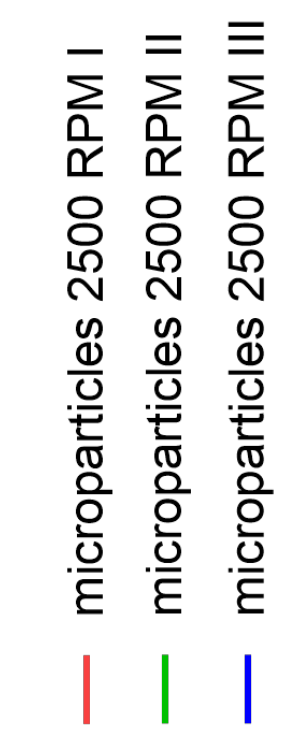

(త)

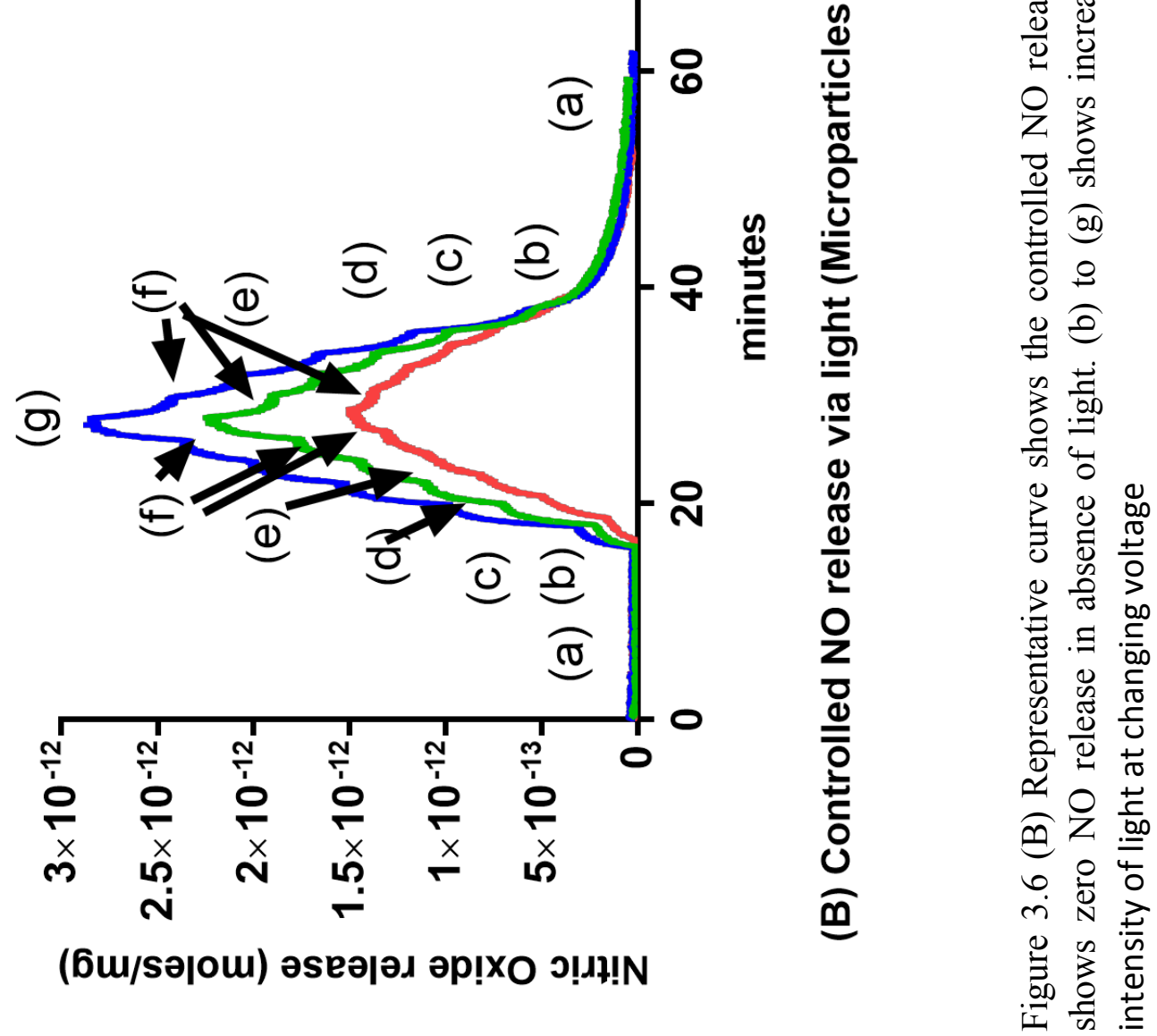




\subsubsection{Effect of particle size on the light initiated controlled NO release between microparticles}

In this study, when the amount of NO released from the microparticle $(500)$ and microparticle $_{(2500)}$ via light were compared, it did not show any significant difference $(p=0.67)$ between the two. The NO release from inside the microparticles through SNAP decomposition occurs mainly through the diffusion. This determines that the amount of NO release depends on the surface area of the microparticles the light has been shined upon. The particles with bigger surface area should release more NO compared to the particles with smaller surface area. However, according to the Fig. 3.7, no significant difference $(\mathrm{p}=0.67)$ has been observed between both microparticles. This may infer that either controlled NO release via light over a given period of time does not depend on the surface area of the microparticle or the size difference between both the microparticles is not significant enough to produce significantly different NO amounts. 


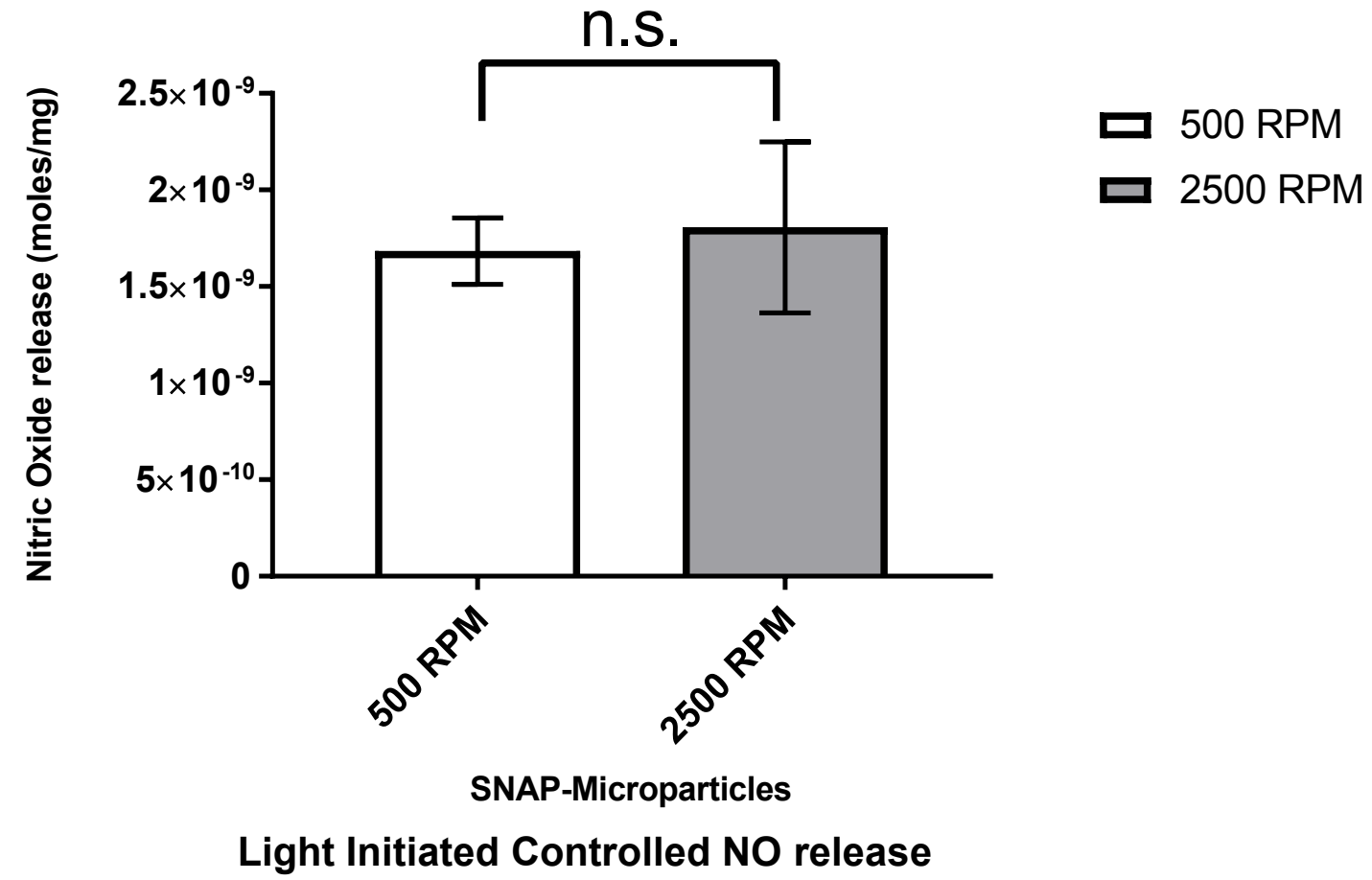

Figure 2.8 Comparison of controlled light initiated NO release from microparticles(500) and microparticles $(2500)$

\subsubsection{Total NO release study via light, Copper (II) ions and ascorbate}

Total NO means the overall amount of NO that can be released or available for release from the SNAP encapsulated in the PLLA microparticles. Total NO release measurements were done using all the triggers responsible for NO production from SNAP. This includes light (photo initiation) using LED, copper (II) ions and ascorbic acid. The NO release measurements were done at room temperature. In Fig. 3.8 (a) and (b), the microspheres were shown to release $\mathrm{NO}$ in the presence of $50 \mu \mathrm{M} \mathrm{CuCl} 2$ and $100 \mathrm{mM}$ ascorbic acid which are SNAP decomposition initiators. In the experimental setup during NO detection, after reaching a plateau by purging out the released $\mathrm{NO}$ for $15 \mathrm{mins}, 0.5 \mathrm{ml}$ of $50 \mu \mathrm{M} \mathrm{CuCl} 2$ and $100 \mathrm{mM}$ ascorbic acid was added to the vial using a syringe which showed an instantaneous release of NO from microparticles. LED light was turned on at $4 \mathrm{~V}$ which acts as an additional factor in NO release. The SNAP-PLLA microparticle showed to follow biphasic 
NO release profile that is an initial burst release and then a slower release period, which is typical for PLGA drug delivery devices (DDDs).

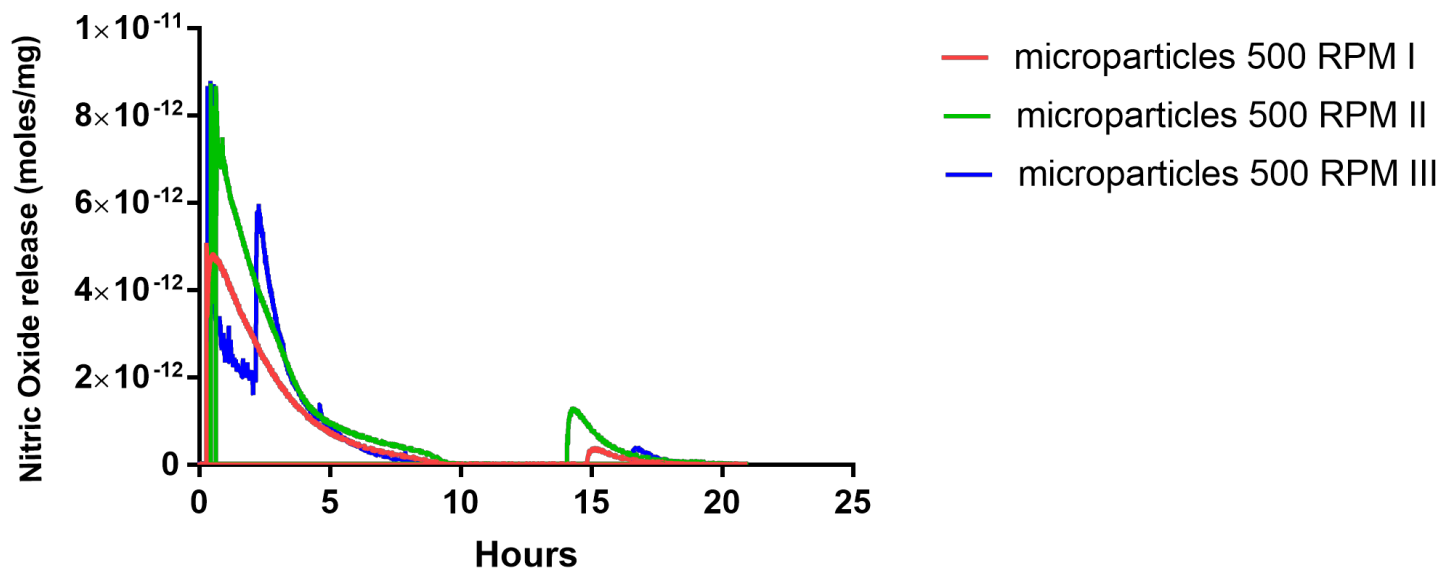

(a) Total NO release (Microparticles 500 RPM)

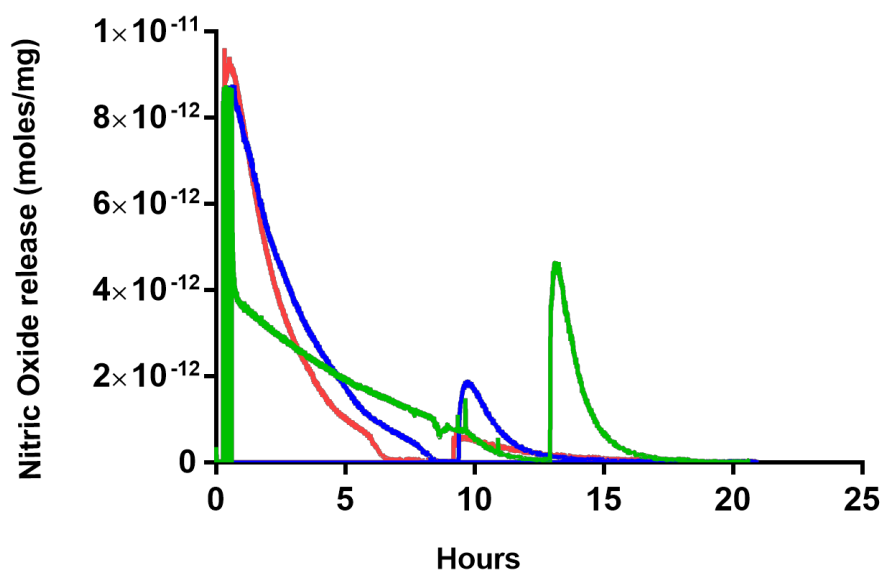

- microparticles 2500 RPM I

- microparticles 2500 RPM II

- microparticles 2500 RPM III

(b) Total NO release (Microparticles 2500 RPM)

Figure 2.9 Total NO release from the (a) microparticle $_{(500)}$ (b) microparticles $_{(2500)}$ using light $(4 \mathrm{~V}), 50 \mu \mathrm{M} \mathrm{CuCl} 2$ and $100 \mathrm{mM}$ ascorbic acid

As shown above, in this study, drug release profile reveals initial burst followed by slower release period. During the initial burst period that ensues over the first two hours, approximately $42.1 \% \pm 7.6$ and $39.2 \% \pm 14.8$ of total $\mathrm{NO}$ measured is released from the SNAP-PLLA microparticle(500) and SNAP-PLLA microparticles(2500) respectively. After the burst release, the drug is released more slowly over next several hours. The 
microparticle $_{(500)}$ and microparticles $(2500)$ showed uninterred $\mathrm{NO}$ release for over 9 hours and 13 hours respectively as seen in Fig. 3.9. After that time, the copper (II) ions in the solution probably got exhausted and another $0.5 \mathrm{ml}$ of $50 \mu \mathrm{M} \mathrm{CuCl} 2$ and $100 \mathrm{mM}$ ascorbic acid was added to check more NO release. It was observed that only light was not enough for further release which may be due to not enough penetration of light into the microparticles. Both microparticles showed extended NO release for approximately 2 hours before stopping more NO release. Addition of another $0.5 \mathrm{ml}$ of $50 \mu \mathrm{M} \mathrm{CuCl} 2$ and $100 \mathrm{mM}$ ascorbic acid did not cause any more NO release which showed no more possibility of NO production from the used microparticles.

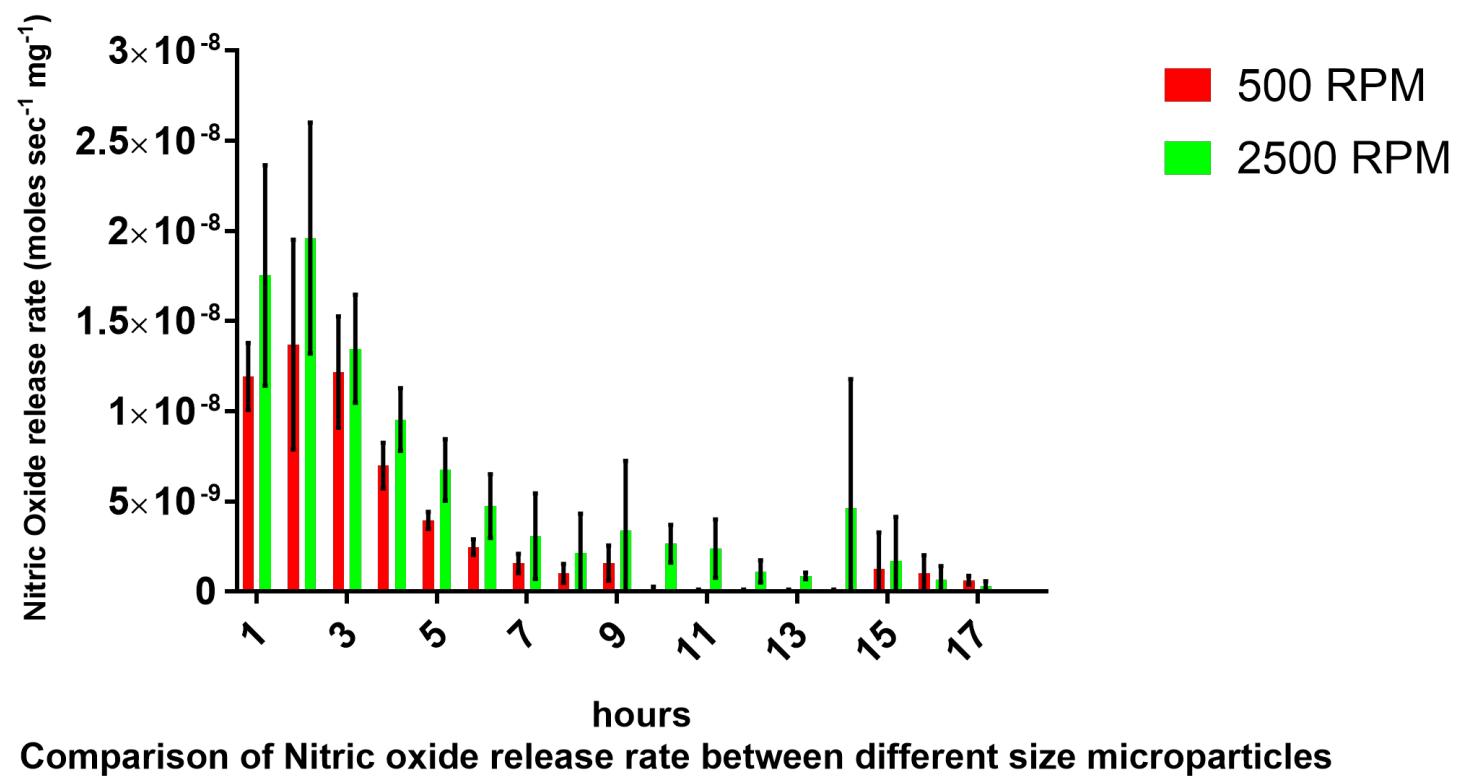

Figure 2.10 comparison of $\mathrm{NO}$ release rate from the microparticle $(500)$ and microparticles $(2500)$

\subsubsection{Effect of particle size on total NO release between microparticles}

As shown in the fig. 3.10 below, using the student's two tail t-test, the comparison of the total amount of NO produced between the microparticle $(500)$ and microparticle $(2500)$ exhibited a statistically significant difference $(\mathrm{p}=0.03)$ in the NO released from both particles. This can infer that particle size affects the amount of release from the particles. The nitric oxide release rate from the microparticle $(2500)$ is also higher compared to microparticle $(500)$ as seen in fig 3.9 above. It is strange to observe more total NO release in 
microparticle $_{(2500)}$ compared to microparticle $(500)$ as both have shown almost similar encapsulation efficiency of SNAP. The reason for this can be either easy availability of more encapsulated SNAP in smaller particles (microparticle $(2500)$ ) or non-availability of some amount of encapsulated SNAP in bigger particles (microparticle $(500)$ ). A difference in NO production quantities due to difference in particle size can be clearly observed.

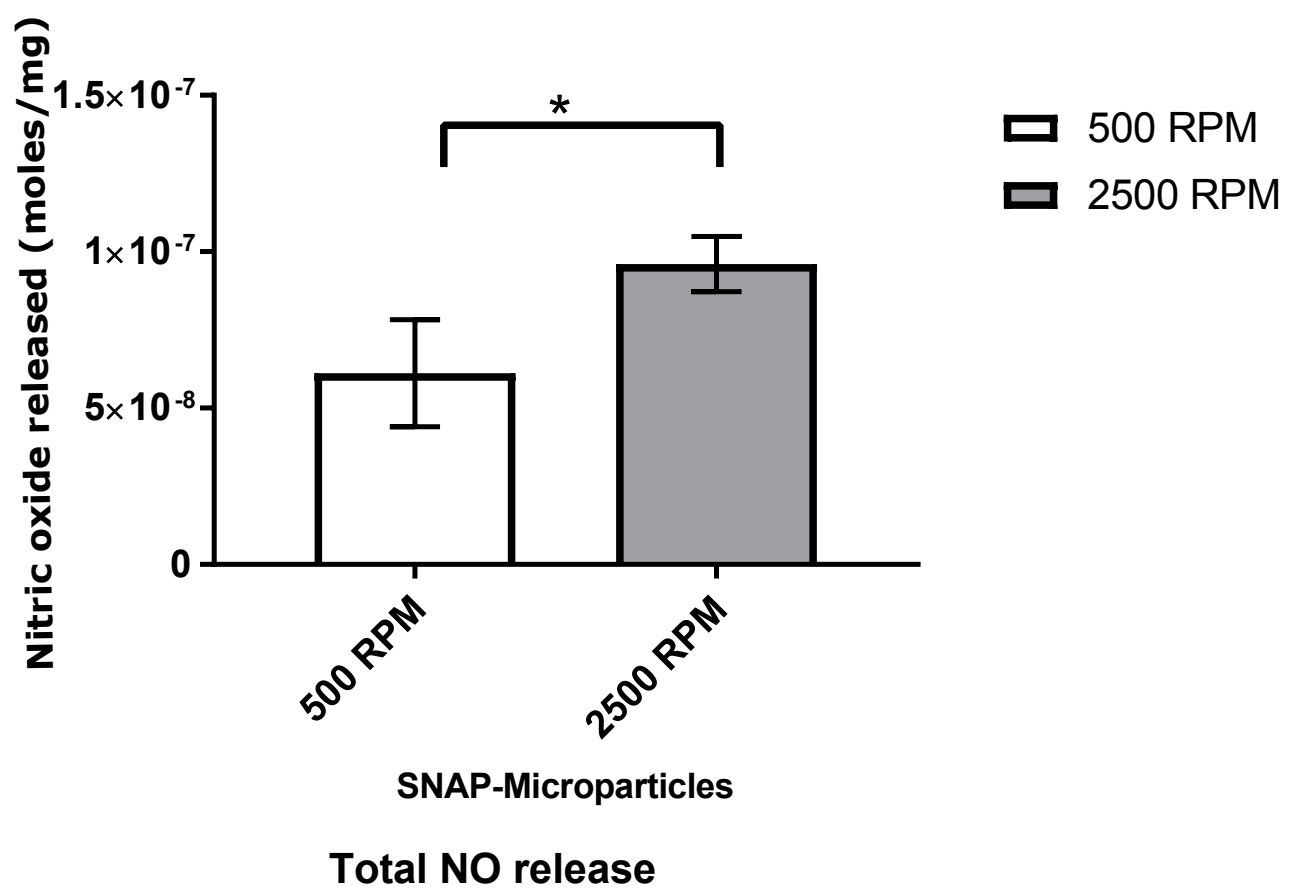

Figure 2.11 Comparison of total NO release from microparticles $(500)$ and microparticles(2500)

\subsection{Discussion}

SNAP (S-Nitroso-N-acetylpenicillamine) loaded PLLA microparticles were prepared using double emulsion (water-in-oil-in -water) emulsion solvent evaporation method. The microparticle system was developed and investigated as a possible nitric oxide delivery system for wound healing application.

\subsubsection{Size and characteristic of microparticle}

SEM image analysis of the microparticles revealed smooth but irregular shape of the microparticles. The microparticles were expected to have a spherical structure but most of 
them especially larger ones had depressions in them. They may have been formed during the air drying process, but changes due to their exposure to the electron beam during SEM imaging is also possible (35). The average size of microparticles ${ }_{(500)}(256 \pm 11.7 \mu \mathrm{m})$ and $\operatorname{microparticles}_{(2500)}(207 \pm 19.7 \mu \mathrm{m})$ was large which can be due to low force and shear required to make them smaller as stirring rate was kept low. Also, homogenization was not done in this method which may be reason for the larger size. The structure underneath the surface may be dense, porous or hollow but any conclusion on the inner structure of microparticles based on these pictures cannot be made. SEM pictures of these same microparticles after full drug release would have been interesting. Many factors that affect the structure of the microparticles but the most important probably is the preparation method (36). Here, water-in-oil-in-water (w/o/w) double emulsion solvent evaporation method is used. In double emulsion process, solvent evaporation significantly affects the structure of the formed particles. Solvent evaporation rate influences the inner structure of the microparticle. Porous structure can collapse easily than dense structure causing early degradation of matrix (37).

\subsubsection{Encapsulation efficiency based on total NO release}

Although encapsulation efficiency was calculated using UV-vis spectroscopy. We also calculated encapsulation efficiency based on total NO release analysis. As reported above, overall amount of NO released by the microparticles was less than the calculated encapsulated efficiency. UV-vis results showed almost equal encapsulation efficiency for both microparticles(500) and microparticles $(2500)$. However, based on total NO release analysis, a difference in encapsulation efficiency was observed with microparticles(2500) showing higher encapsulation than microparticles(500). This can be due to significant difference $(\mathrm{p}=0.02)$ in microparticle sizes. 
Table 3 Comparison of average total nitric oxide release and encapsulation efficiency from SNAP-PLLA microparticle $(500)$ and SNAP-PLLA microparticle $(2500)$

\begin{tabular}{|l|l|l|}
\hline Composition & Average total NO release \pm s.d. & Encapsulation efficiency (\%) \\
\hline $\begin{array}{l}\text { SNAP-PLLA } \\
\text { microparticle }_{(500)}\end{array}$ & $6.11 \pm 1.71 \times 10^{-8}$ & $14.79 \% \pm 4.14$ \\
\hline $\begin{array}{l}\text { SNAP-PLLA } \\
\text { microparticle }(2500)\end{array}$ & $8.43 \pm 2.92 \times 10^{-8}$ & $23.25 \% \pm 2.14$ \\
\hline
\end{tabular}

There could be another reason for this difference in overall NO release even though no difference was shown in encapsulation efficiency using UV-vis. This could be due to possible bonding of SNAP to the PLLA polymer through hydrogen bond. It is possible that SNAP is more closely bound to PLLA in larger microparticles compared to smaller ones.

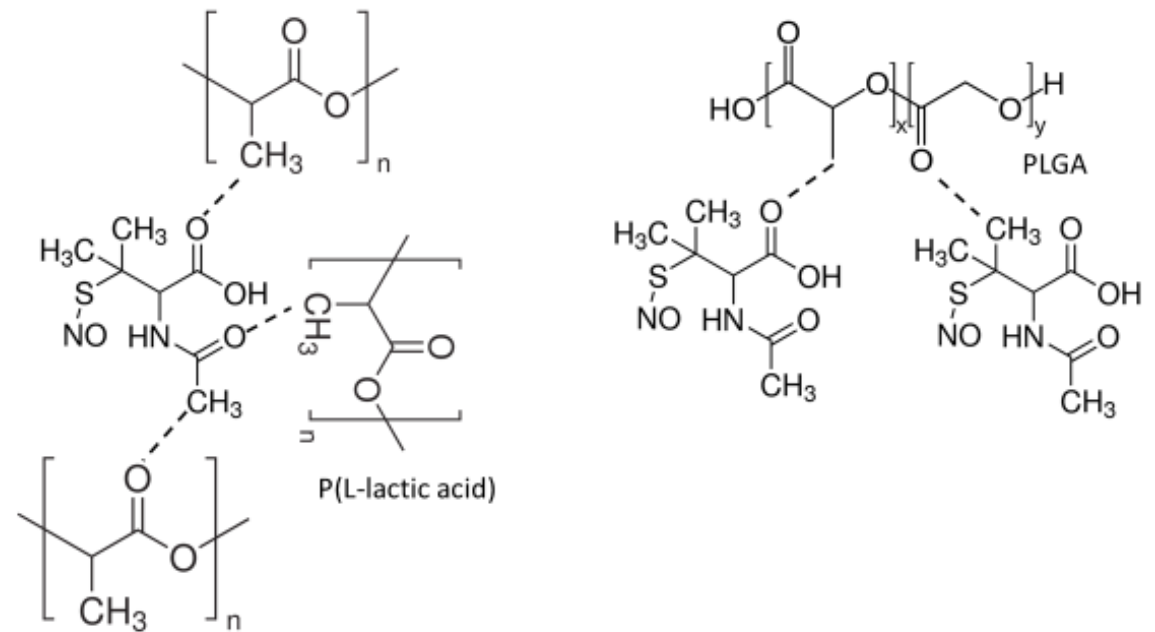

Figure 2.12 Possible mechanism of SNAP bonding to PLLA by hydrogen bond. 


\subsubsection{Low encapsulation efficiency}

The drug encapsulation efficiency is an essential factor in drug delivering device as it determines how much drug can be loaded and released. As the double emulsion solvent evaporation was used in this study, constituting two major phases- dispersed phase (an inner aqueous phase containing the drug (SNAP) and polymer organic phase) and continuous phase (emulsifier containing aqueous phase). The NO donor SNAP used in this study is hydrophilic which causes its diffusion in to the continuous phase from the dispersed phase resulting in low encapsulation efficiency. To prevent this, double emulsion method was used as it prevents the escape of SNAP from dispersed phase in to the continuous phase by first formation of primary emulsion. However, still some loss of SNAP occurred. The SNAP also degrades due to the presence of even traces of metal ions especially copper. The $\mathrm{Cu}$ metal ions were chelated using metal chelators chelex and cyclam to prevent unwanted NO release due to decomposition of SNAP by $\mathrm{Cu}+$. Additionally, SNAP is stable at acidic $\mathrm{pH}$ (38). Thus, the $\mathrm{pH}$ of the continuous phase plays an important role in stabilizing the drug to be encapsulated which in turn can increases its encapsulation in the microparticle (19). The continuous phase which is PVA in water turns basic due to chelex addition. It is acidified by adding $\mathrm{HCl}$. This helps in preventing SNAP degradation in the developing microparticles while preparation. However, even after taking such steps, the encapsulation efficiency was low. There can be several reasons for low encapsulation efficiency such as low polymer concentration, high dispersed phase to continuous phase ration and slow solvent removal rate (39). The SNAP in this study was first dissolved in DMSO (dimethyl sulfoxide) during formation of primary emulsion as solubility of SNAP in DMSO is higher than water. The DMSO could have caused SNAP degradation or its inability to form a stable primary emulsion could be reason for low encapsulation. 


\subsubsection{NO release profile and burst release}

In vitro drug release profile from the SNAP loaded microparticles followed a bi-phasic release profile that is initial burst release within few hours followed by slower release phase. This is a characteristic of PLA microparticles particularly with small dimeter (4042). Burst release is a fast release phase in the beginning of the release period. There are several reasons for fast $\mathrm{NO}$ release from the microparticles. The amount of $\mathrm{NO}$ which is released in this study during the burst release could include non-encapsulated SNAP, SNAP located near the surface as well as SNAP adhered to the surface of microparticles. Although during the processing, the microparticles were washed to remove unencapsulated SNAP, some amount can remain in the final product. The most important factor affecting the properties of microparticles is the preparation method (36). We cannot directly compare the microparticles prepared in this study with microspheres prepared by Lautner et al. because of different preparation methods themselves affects microparticle properties. Parameters such as polymer concentration, ratio of dispersed to continuous phase in the emulsion preparation phase poses challenges to microparticle preparation can all influence properties of microparticles. in the literature, the microspheres made using solid-in-wateroil emulsion solvent evaporation method with free acid terminated PLGA polymer (RG $503 \mathrm{H}$ ) showed NO release for typically 10-14 days while 30 days in case of ester capped terminus PLGA (RG 504) microspheres (32). The diameter of those microspheres was in the range of 50-60 $\mu \mathrm{m}$. The average size of particles i.e. microparticles(500) $(256.7 \pm 11.7$ $\mu \mathrm{m})$ and $\operatorname{microparticles}_{(2500)}(207.4 \pm 19.7 \mu \mathrm{m})$ in our study are comparatively very large. Also, in this study, PLLA polymer was used for NO delivery not PLGA as degradation rate of PLLA is expected to be much slower due to absence of glycolic acid in the matrix.

The second reason can be size of the microparticles. Diffusion is a crucial factor in drug release from polymeric microparticles. Usually when the size of the drug delivery device (microparticle) increases, diffusion pathway becomes longer which in turn slows drug release (43). Smaller microparticles would have a high surface area: volume ratio and shorter diffusion distance that allows more rapid release. However, drug distribution within polymer matrix is also affected by microparticle size (41) which is the third reason that is 
heterogeneous drug distribution. Drug release profile is affected by the location of the drug in the microparticle. The main reason for burst release is surface associated drug (39). The method used in this study should enable the homogenous drug distribution in polymer matrix as drug and polymer are completely dissolved into the solvent. Therefore, drug release becomes faster with a decrease in diameter of microparticles as well as also indirectly affected by increased partitioning of the drug to the surface with increasing size and more rapid polymer degradation in larger microparticles. Interestingly, in this study microparticles have larger diameters and wider size distribution. Drug release period became longer when the diameter was decreased (diameter microparticle(2500) $_{\text {< }}$

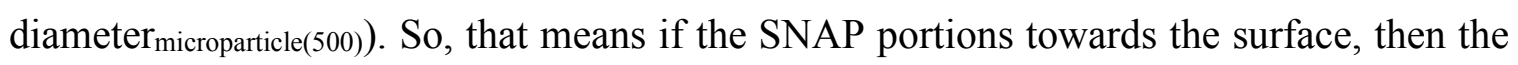
diffusion distance decreases in larger microparticles which expects to increase NO release rate. The effect of diffusion due to size is superimposed by the effect of the drug distribution. Along with this, increased degradation rate of larger microparticles can also slightly increase release rate.

Fourth probable reason is inner porosity. As described earlier, the preparation method affects the structure of the microparticles. There are contrast in literature about effect of solvent evaporation rate. According to some, the faster solvent evaporation rate means more porous structure of the microparticles $(36,44)$. While some show that in solvent evaporation method, more porous structure formed due to diffusion of aqueous phase in to dispersed phase cause by low solvent evaporation rate (39). Porous matrix has faster drug release rate compared to dense matrix microparticles. Fifth reason can be polymer-polymer interactions. In this study, PVA has been as an emulsifier which many affect the drug release properties of the microparticles. PVA may form a layer around formed microparticles catalyzing degradation as it can act as a barrier to prevent diffusion of acidic degradation product of PLA out from the microparticles (45). Low molecular weight drugs are likely to have burst release profiles because of their small size (46). Nitric oxide is a small molecule which can be the reason for burst release.

All this shows that, so many factors affect the drug release from biodegradable microparticles as predicting the drug release is difficult. However, several methods exist 
to modify release profile such as coating microparticle with other more slowly degrading polymer (47).

\subsection{Summary}

In this study, the PLLA microparticles encapsulated with NO donor SNAP were prepared using double emulsion solvent evaporation $(\mathrm{W} / \mathrm{O} / \mathrm{W})$. The microparticles of varied sizes (microparticle $(500)$ and microparticle $(2500)$ ) were prepared using different stirring speeds. Considering all the experimental data collected, these PLLA microparticles encapsulating SNAP were shown to release nitric oxide in a controllable manner on exposure to the light. At different light intensities, SNAP-PLLA microparticles were shown to release different amount of nitric oxide. The SNAP-PLLA microparticles were also experimentally tested to show NO release in the presence of copper (II) and ascorbate. The microparticle $(500)$ demonstrated an average total NO release of $6.11 \pm 1.71 \times 10^{-08}$ for 9 hours and $\operatorname{microparticle}_{(2500)}$ exhibited average total NO release of $8.43 \pm 2.92 \times 10^{-08}$ for 13 hours. These reported microparticles can be injected into the wound to accelerate healing or can be incorporated into the hydrogel to be used as wound dressing.

\subsection{References}

1. Moncada S, Higgs E. The discovery of nitric oxide and its role in vascular biology. British journal of pharmacology. 2006;147(S1).

2. Hill BG, Dranka BP, Bailey SM, Lancaster JR, Darley-Usmar VM. What part of NO don't you understand? Some answers to the cardinal questions in nitric oxide biology. Journal of Biological Chemistry. 2010;285(26):19699-704.

3. Isenberg JS, Ridnour LA, Espey MG, Wink DA, Roberts DA. Nitric oxide in wound-healing. Microsurgery. 2005;25(5):442 -51.

4. Rizk M, Witte MB, Barbul A. Nitric oxide and wound healing. World journal of surgery. 2004;28(3):301-6. 
5. Lancaster J. A tutorial on the diffusibility and reactivity of free nitric oxide. Nitric Oxide. 1997;1(1):18-30.

6. Carpenter AW, Schoenfisch MH. Nitric oxide release: Part II. Therapeutic applications. Chemical Society Reviews. 2012;41(10):3742-52.

7. Wang PG, Xian M, Tang X, Wu X, Wen Z, Cai T, et al. Nitric oxide donors: chemical activities and biological applications. Chemical reviews. 2002;102(4):1091-134.

8. Coneski PN, Rao KS, Schoenfisch MH. Degradable nitric oxide-releasing biomaterials via post-polymerization functionalization of cross-linked polyesters. Biomacromolecules. 2010;11(11):3208-15.

9. Wo Y, Brisbois EJ, Bartlett RH, Meyerhoff ME. Recent advances in thromboresistant and antimicrobial polymers for biomedical applications: just say yes to nitric oxide (NO). Biomaterials science. 2016;4(8):1161-83.

10. Zhao H, Serrano MC, Popowich DA, Kibbe MR, Ameer GA. Biodegradable nitric oxide-releasing poly (diol citrate) elastomers. Journal of Biomedical $M$ aterials Research Part A. 2010;93(1):356-63.

11. Wang Y, Kibbe MR, Ameer GA. Photo-crosslinked biodegradable elastomers for controlled nitric oxide delivery. Biomaterials science. 2013;1(6):625-32.

12. Pulfer SK, Ott D, Smith DJ. Incorporation of nitric oxide-releasing crosslinked polyethyleneimine microspheres into vascular grafts. Journal of Biomedical Materials Research Part A. 1997;37(2):182-9.

13. Jeh H, Lu S, George S. Encapsulation of PROLI/NO in biodegradable microparticles. Journal of microencapsulation. 2004;21(1):3-13.

14. Zhang H, Annich GM, Miskulin J, Stankiewicz K, Osterholzer K, Merz SI, et al. Nitric oxide-releasing fumed silica particles: synthesis, characterization, and biomedical application. Journal of the American Chemical Society. 2003;125(17):5015-24.

15. Rothrock AR, Donkers RL, Schoenfisch MH. Synthesis of nitric oxide-releasing gold nanoparticles. Journal of the American Chemical Society. 2005;127(26):93623.

16. Shin JH, Metzger SK, Schoenfisch MH. Synthesis of nitric oxide-releasing silica nanoparticles. Journal of the American Chemical Society. 2007;129(15):4612-9.

17. Stasko NA, Schoenfisch MH. Dendrimers as a scaffold for nitric oxide release. Journal of the American Chemical Society. 2006;128(25):8265-71. 
18. Carpenter AW, Reighard KP, Saavedra JE, Schoenfisch MH. O 2-Protected diazeniumdiolate-modified silica nanoparticles for extended nitric oxide release from dental composites. Biomaterials science. 2013;1(5):456-9.

19. Yoo JW, Lee JS, Lee CH. Characterization of nitric oxide-rel easing microparticles for the mucosal delivery. Journal of Biomedical Materials Research Part A. 2010;92(4):1233-43.

20. Nurhasni H, Cao J, Choi M, Kim I, Lee BL, Jung Y, et al. Nitric oxide-releasing poly (lactic-co-glycolic acid)-polyethylenimine nanoparticles for prolonged nitric oxide release, antibacterial efficacy, and in vivo wound healing activity. International journal of nanomedicine. 2015;10:3065.

21. Coneski PN, Schoenfisch MH. Competitive formation of N-diazeniumdiolates and $\mathrm{N}$-nitrosamines via anaerobic reactions of polyamines with nitric oxide. Organic letters. 2009;11(23):5462-5.

22. Haitham A-SD, Ferro A. S-Nitrosothiols: a class of nitric oxide-donor drugs. Clinical science. 2000;98(5):507-20.

23. Szaciłowski K, Stasicka Z. S-nitrosothiols: materials, reactivity and mechanisms. Progress in Reaction Kinetics and Mechanism. 2001;26(1):1-58.

24. Williams DLH. The chemistry of S-nitrosothiols. Accounts of chemical research. 1999;32(10):869-76.

25. Gierke GE, Nielsen M, Frost MC. S-Nitroso-N-acetyl-D-penicillamine covalently linked to polydimethylsiloxane (SNAP-PDMS) for use as a controlled photoinitiated nitric oxide release polymer. Science and technology of advanced materials. 2011;12(5):055007.

26. Brisbois EJ, Handa H, Major TC, Bartlett RH, Meyerhoff ME. Long-term nitric oxide release and elevated temperature stability with S-nitroso-Nacetylpenicillamine (SNAP)-doped Elast-eon E2As polymer. Biomaterials. 2013;34(28):6957-66.

27. Handa H, Major TC, Brisbois EJ, Amoako KA, Meyerhoff ME, Bartlett RH. Hemocompatibility comparison of biomedical grade polymers using rabbit thrombogenicity model for preparing nonthrombogenic nitric oxide releasing surfaces. Journal of Materials Chemistry B. 2014;2(8):1059-67.

28. de Oliveira MG, Shishido SM, Seabra AB, Morgon NH. Thermal stability of primary S-nitrosothiols: roles of autocatalysis and structural effects on the rate of nitric oxide release. The Journal of Physical Chemistry A. 2002;106(38):8963-70. 
29. Wo Y, Li Z, Brisbois EJ, Colletta A, Wu J, Major TC, et al. Origin of long-term storage stability and nitric oxide release behavior of carbosil polymer doped with S-nitroso-N-acetyl-D-penicillamine. ACS applied materials \& interfaces. 2015;7(40):22218-27.

30. Frost MC, Meyerhoff ME. Synthesis, characterization, and controlled nitric oxide release from S-nitrosothiol-derivatized fumed silica polymer filler particles. Journal of Biomedical Materials Research Part A. 2005;72(4):409-19.

31. Stasko NA, Fischer TH, Schoenfisch MH. S-nitrosothiol-modified dendrimers as nitric oxide delivery vehicles. Biomacromolecules. 2008;9(3):834-41.

32. Lautner G, Meyerhoff ME, Schwendeman SP. Biodegradable poly (lactic-coglycolic acid) microspheres loaded with S-nitroso-N-acetyl-D-penicillamine for controlled nitric oxide delivery. Journal of Controlled Release. 2016;225:133-9.

33. Babich H, Zuckerbraun HL, Hirsch ST, Blau L. In vitro Cytotoxicity of the Nitric Oxide Donor, S-Nitroso- N- acetyl - peraciUamine, towards Cells from Human Oral Tissue. Basic \& Clinical Pharmacology \& Toxicology. 1999;84(5):218-25.

34. Levitskaia TG, Creim JA, Curry TL, Luders T, Morris JE, Woodstock AD, et al. Evaluation of Cuprimine ${ }^{\circledR}$ and Syprine ${ }^{\circledR}$ for decorporation of $60 \mathrm{Co}$ and $210 \mathrm{Po}$. Health physics. 2010;98(3):471-9.

35. Loo J, Ooi CP, Boey F. Degradation of poly (lactide-co-glycolide)(PLGA) and poly (L-lactide)(PLLA) by electron beam radiation. Biomaterials. 2005;26(12):1359-67.

36. Yushu H, Venkatraman S. The effect of process variables on the morphology and release characteristics of protein-loaded PLGA particles. Journal of applied polymer science. 2006;101(5):3053-61.

37. Martínez-Sancho C, Herrero-Vanrell Ro, Negro Sa. Study of gamma-irradiation effects on aciclovir poly (D, L-lactic-co-glycolic) acid microspheres for intravitreal administration. Journal of controlled release. 2004;99(1):41-52.

38. Singh RJ, Hogg N, Joseph J, Kalyanaraman B. Mechanism of nitric oxide release from S-nitrosothiols. Journal of Biological Chemistry. 1996;271(31):18596-603.

39. Yeo Y, Park K. Control of encapsulation efficiency and initial burst in polymeric microparticle systems. Archives of pharmacal research. 2004;27(1):1.

40. Berchane N, Carson K, Rice-Ficht A, Andrews M. Effect of mean diameter and polydispersity of PLG microspheres on drug release: Experiment and theory. International journal of pharmaceutics. 2007;337(1):118-26. 
41. Berkland C, Kim KK, Pack DW. PLG microsphere size controls drug release rate through several competing factors. Pharmaceutical research. 2003;20(7):1055-62.

42. Fredenberg S, Wahlgren M, Reslow M, Axelsson A. The mechanisms of drug release in poly (lactic-co-glycolic acid)-based drug delivery systems - a review. International journal of pharmaceutics. 2011;415(1):34-52.

43. Siepmann J, Göpferich A. Mathematical modeling of bioerodible, polymeric drug delivery systems. Advanced drug delivery reviews. 2001;48(2):229-47.

44. Freitas S, Merkle HP, Gander B. Microencapsulation by solvent extraction/evaporation: reviewing the state of the art of microsphere preparation process technology. Journal of controlled release. 2005;102(2):313-32.

45. Panyam J, Dali MM, Sahoo SK, Ma W, Chakravarthi SS, Amidon GL, et al. Polymer degradation and in vitro release of a model protein from poly (D, Llactide-co-glycolide) nano-and microparticles. Journal of Controlled Release. 2003;92(1):173-87.

46. Huang X, Brazel CS. On the importance and mechanisms of burst release in matrixcontrolled drug delivery systems. Journal of controlled release. 2001;73(2):121-36.

47. Berkland C, Cox A, Kim KK, Pack DW. Three-month, zero- order piroxicam release from monodispersed double-walled microspheres of controlled shell thickness. Journal of Biomedical Materials Research Part A. 2004;70(4):576-84. 


\section{Particles in hydrogel system}

\subsection{Introduction}

There is a need for developing new wound dressings which can improve wound healing process, making it less painful and help in reconstructing the skin structure and function in a brief period (1,2). Among all the wound dressers introduced so far, hydrogels have been crucial for skin regeneration because of their intrinsic properties which mimic the extracellular matrix (ECM) and have the potential for cell proliferation, adhesion and growth during tissue regeneration $(3,4)$. Hydrogels have high patient acceptability as they aid in moist healing and provide coolness to the surface of the wound when applied to the wound site leading to major pain reduction $(5,6)$.

As described above, $\mathrm{NO}$ is an important signaling molecule involved in many physiological processes such as immune responses, wound healing, vascular and neurological functions $(7,8)$. NO has short half-life, short diffusion distance and its therapeutic effects are dose, duration and location dependent (9). Therefore, on demand controlled delivery biologically active NO is very critical for any NO based effective therapies. Several hydrogels based therapies have been studied by researchers to provide effective NO delivery. One of the approach is $\beta$-galactosidase-mediated release of nitric oxide (NO) from hydrogels (10). Zhao et al. covalently linked $\beta$-galactosidase-sensitive NO donors ( $\beta$-gal-NONOate) to the polymer backbone to develop an injectable chitosan hydrogel system (11). The enzymatic activity of hydrogel $\beta$-galactosidase controls the NO release in micromolar concentrations by hydrolyzing the glycosidic bonds. These materials exhibited no decomposition with storage stability for over six months at room temperature. It was reported that local delivery of NO by utilizing endogenous $\beta$-galactosidase activity lead to enhanced proliferation of endothelial cells and angiogenesis stimulation in vivo. An enzyme mediated NO releasing system lacks a fine control of dose and duration even though they demonstrate targeted NO delivery on targeted sites. Hence, dosage control and delivery rate of $\mathrm{NO}$ are the main challenges in developing NO based therapies. 
Three NO releasing hydrogels were synthesized by Bohl and West with two containing diazeniumdiolate moieties and one with covalently linked S-nitrosothiol functionalities (12). Polyethylene glycol N-hydroxysuccinimide monoacrylate (ACRL-PEG-NHS) was reacted with poly-L-lysine to form a polyethylene glycol (PEG)-poly-L-lysine (PEG-Lys5NO) hydrogel. In this NO releasing hydrogel, the diazeniumdiolate was formed of free amines of lysine residues followed by photocrosslinking with PEG-diacrylate. Another hydrogel (PEG-DETA-NO) was prepared in equivalent manner with diethylenetriamine (DETA) as an amine source formed by reacting with ACRL-PEG-NHS and photocrosslinking. PEG-S-nitrosocysteine hydrogel was formed by reacting L-cysteine with ACRL-PEG-NHS to form thiol-containing PEG species which were nitrosated in nitrous acid and photopolymerized. These hydrogels showed sufficient NO release for smooth muscle cell (SMC) growth inhibition and reduction in platelet adhesion. Shishido et al. incorporated GSNO and S-nitroso-N-acetylcysteine (SNAC) into poly (ethylene oxide)-poly (propylene oxide)-poly (ethylene oxide) (PEO-PPO-PEO) copolymer hydrogel which showed slow NO release rate and prolong NO release lifetime of material (13). An increase in blood flow indicating vasodilation of the underlying blood vessels was observed when these materials were applied topically to the skin (14). Vercelino et al. developed a S-nitrosoglutathione incorporated topical pluronic F127 hydrogel for treating pain and impaired dermal blood flow (15). In another study by Schanuel et al. functionalized PVA films with SNO groups and combined them with pluronic F127 hydrogel. The hydrogel layer prevents the burst release of NO from film and their combination helps in accelerating wound contraction and inflammatory phase and decrease wound gap (16). A thermosensitive Pluronic F68 branched with polyethylenimine (BPEI) containing N-diazeniumdiolates (NONOates) was developed by Park et al. The developed F68-BPEI-NONOates showed effective antibacterial activity with a good biocompatibility and low cytotoxicity (17). A PEG based ointment incorporated with nitric oxide releasing biocompatible Pluronic F127, branched polyethylenimine and 1-substituted diazen-1-ium1, 2-diolates was developed by Kang et al. The ointment demonstrated a slow NO release, accelerated wound closure with heightened re-epithelialization, collagen deposition, and in vivo blood vessel formation (18). Similarly, a thermosensitive Pluronic F127, branched 
polyethylenimine (BPEI), and diazeniumdiolates (NONOates) was developed as a novel gel system. This F127-BPEI-NONOates system displayed increase in endothelial cell proliferation and reduction of smooth muscle cell proliferation (19).

The Chitosan and agarose interactions permits the hydrogel production which is capable of gelling because of polymer interactions within a desired target site. The combination hydrogel of chitosan and agarose has shown to shown to influence neuron morphology, help in neuron adhesion as well as enhance neural cell attachment and differentiation (20).

The hydrogel has also demonstrated an adequate wound healing environment by keeping the wound moist and hydrated and preventing any bacterial infection (21). The hydrogel exhibited to prevent water loss and wound dehydration and allowing adequate nutrient, oxygen and carbon dioxide exchange at the wound site.

In this study, NO oxide releasing PLLA microparticles are incorporated in to a thermosensitive deacetylated chitosan-agarose to explore their NO oxide releasing capabilities. The wound healing and antibacterial properties of NO combined with the gelling and wound dressing properties of chitosan-agarose hydrogel can be greatly explored for appropriate wound healing.

\subsection{Material and method}

Low molecular weight chitosan (mol wt. 50,000-190,000 Da) was obtained from SigmaAldrich (St. Louis, MO). Agarose was purchased from SeaPrep (Seattle, WA). L- (+)Ascorbic Acid used for experiments was purchased from J.T Baker ( $>=99.5 \%$ ) (Center Valley, PA, USA).

\subsubsection{Preparation of SNAP loaded microparticles and chitosan-agarose hydrogel composite}

Chitosan-agarose hydrogel was prepared using a simple procedure described by Cao et al (20). The schematic representation of the production of chitosan-agarose hydrogel embedded with snap-loaded microparticle is shown in Fig. 4.1. Briefly, 2\% (w/w) chitosan 
(30 mg) was first dissolved to the $2.5 \%(\mathrm{v} / \mathrm{v})$ acetic acid solution $(50 \mu 1$ acetic acid in 1950 $\mu 1$ chelexed water). Chelex treated water was used to prevent any copper ion contamination. $5 \mathrm{mg}$ of SNAP loaded microparticles were added to the chitosan solution. The chitosan microparticle suspension was sonicated for $2 \mathrm{~min}$ and then magnetically stirred for an hour. The chitosan-microparticles suspension was stirred to remove any microparticle aggregates. Microparticles should be homogenously distributed when introduced into the hydrogel. Agarose was then added to this suspension and dissolved by heating in a $60{ }^{\circ} \mathrm{C}$ water bath.

Polypropylene cylinders (1.2 cm diameter) were used as a mold to prepare the hydrogels. After pouring the chitosan-agarose-microparticle solution in to the cut syringe, they were covered with the parafilm to prevent any moisture evaporation and left at $4{ }^{\circ} \mathrm{C}$ for complete gelation. Total gelation time depends on the volume of the hydrogel. Usually, this hydrogel composition can gel within $20 \mathrm{~min}$ at $4{ }^{\circ} \mathrm{C}$, however, large gels can take a longer time. Therefore, the prepared hydrogels were left to gelate overnight. Blank hydrogels were also prepared using the same method as the hydrogel microparticle composite. The blank hydrogel is the hydrogel with same formulation as the composite gel without microparticles. 


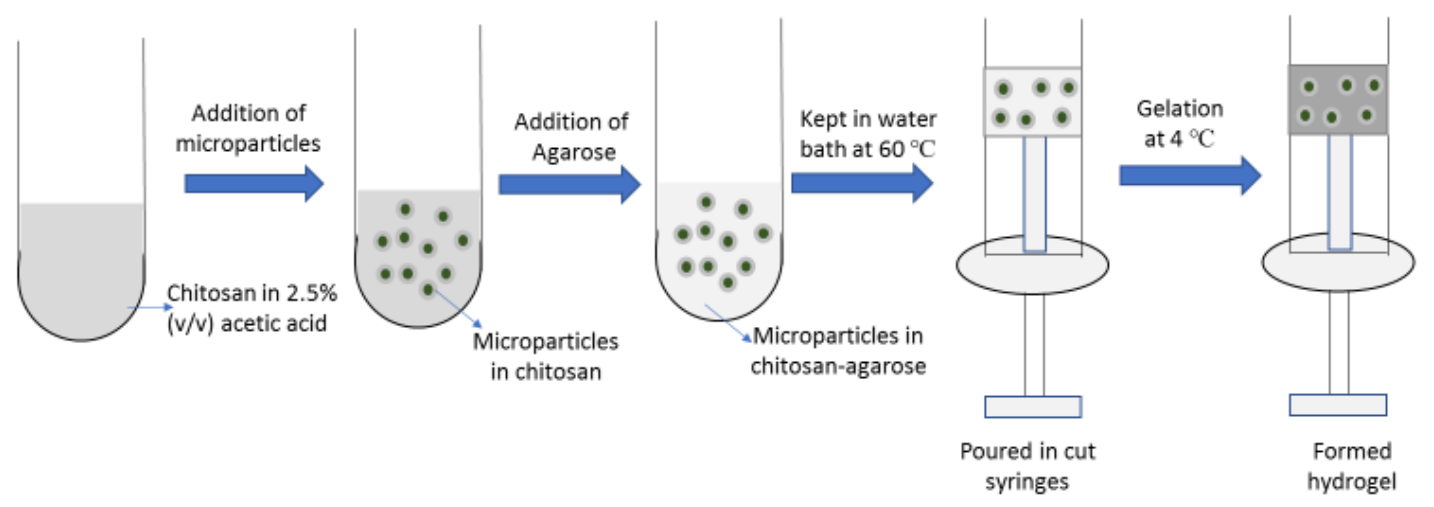

Figure 3.1 Preparation of microparticle incorporated chitosan-agarose hydrogel

\subsubsection{Preparation of ascorbic acid chitosan-agarose composite hydrogel}

Ascorbic acid loaded chitosan-agarose hydrogel was formulated using the same method as described above. In this case, instead of microparticles, $100 \mathrm{mM}$ of ascorbic acid was added to the chitosan-acetic acid solution and dissolved. To avoid possibility of NO release from any copper ion present in water, chelexed water was used. NO Then agarose was added and dissolved in a $60{ }^{\circ} \mathrm{C}$ wate bath. This ascorbic acid composite hydrogel is poured in the syringe mold and gelated overnight at $4{ }^{\circ} \mathrm{C}$.

\subsubsection{In vitro nitric oxide release study of Hydrogel-microparticles using Nitric Oxide Analyzer}

The NO release measurements were quantified using an ozone based chemiluminescence detection with Sievers 280i Nitric Oxide Analyzer (NOA) (GE Instruments, Boulder, CO) using $200 \mathrm{ml} / \mathrm{min}$ flow rate, with both house nitrogen as the sweep gas. Nitrogen was used as a sweep gas for carrying NO into NOA. 
Total NO release from hydrogel-microparticle composite was determined separately using light and ascorbic acid hydrogel.

\subsubsection{Total NO release using light}

For the total NO release from hydrogel-microparticle composite using light, hydrogel microparticles composite was placed in an amber vial and illuminated with a $470 \mathrm{~nm}$ LED (VAOL-5GSBY4, Mouser electric, Mansfield, TX) at full intensity at voltage (12V). The NO release was measured until all the NO was released from the composite.

\subsubsection{Total NO release using ascorbic acid hydrogel system}

Another method used for measuring the total NO release from the composite was using ascorbic acid hydrogel composite. Hydrogel-microparticle composite was placed in an amber vial with a magnetic stir bar and then ascorbic acid hydrogel composite was placed above it. In this case too, the NO release was measured until all the NO was released from the composite.

Both NO release experiments were performed at room temperature. The NO levels in ppb unit were converted to moles sec-1 mg-1 unit using the NOA instrument constant that was determined by quantitative reduction of a known quantity of nitrite. Integration of NO release rate curves for given period was done to calculate the amount of NO released in both experiments.

\subsubsection{Statistical analysis}

The statistical analysis of the data was done using MS-excel 2016. All the data were shown as the means $( \pm \mathrm{SD})$. Two-tailed homoscedastic student's t-test was used to statistically analyze the data. The $p$ value $(<0.05)$ was considered significant. The graphs were made using the GraphPad Prism version 7.00 for Windows, GraphPad Software (La Jolla, California, USA) (www.graphpad.com). 


\subsection{Result}

\subsubsection{Microparticle Hydrogel Composite}

This hydrogel-microparticle composite combining SNAP loaded PLLA microparticles incorporated in chitosan-agarose hydrogel was designed with the aim of developing a wound dressing with accelerating wound healing properties and prolonged NO release period. In theory, the chitosan-agarose hydrogel can act as a hemostatic agent to accelerate the process of wound healing and the NO release from microparticles can act antibacterial agent to prevent infection and biofilm production. Hydrogel microparticle composites were prepared with the method described in chapter (20). During hydrogel preparation, microparticles are first made into microparticle suspension in $2.5(\mathrm{v} / \mathrm{v}) \%$ acetic acid solution which is stirred for $1 \mathrm{~h}$. It was done to obtain homogeneous distribution of microparticles in hydrogel, microparticle suspension has to be homogenous without large microparticle aggregates. Preparation of microparticle suspension in acetic acid prevents the degradation of SNAP in the microparticles as SNAP is more stable at acidic $\mathrm{pH}$. It also helps in dissolving chitosan as it is insoluble in water. Even though SNAP loaded PLLA microparticles do not form homogenous suspension with water easily due to large microparticle size, they were just stirred for $1 \mathrm{~h}$ to prevent SNAP degradation in suspension. In this study, the hydrogel preparation was done according to protocol developed by Cao et al. and the gel formulation for composite was chosen from the literature. The agarose concentration was maintained at $1(\mathrm{w} / \mathrm{v}) \%$ which has shown to support the best neurite outgrowth from chick dorsal root ganglion (DRG) (22). Hydrogels with $188 \mu \mathrm{g} / \mathrm{mL}$ of chitosan have exhibited a bactericidal activity. A bactericidal activity was noticed for hydrogels containing more than $188 \mu \mathrm{g} / \mathrm{mL}$ of chitosan. $1.5 \%$ (w/v) \% of chitosan was chosen in this study as it has shown to improve wound healing process and prevent bacterial infection at wound site (21). The amount of microparticle chosen to be introduced in the composite was $5 \mathrm{mg}$ which is the same amount used for in vivo testing of microparticles. 


\subsubsection{Morphology of hydrogel-microparticle composite}

Visual appearance of the hydrogel-microparticle composite and control hydrogel without microparticles is illustrated in the fig.5.1. The shape of both composite gel (microparticlehydrogel) and the control gel (only hydrogel) is regular cylinder shape which is due to the shape of the mold in which they are prepared. Color of the composite gel is white and translucent with visible embedded microparticles. In comparison, control hydrogel is throughout transparent due to absence of microparticles. Microparticles are visible in the hydrogel because of their bigger size. Majority of the microparticles have diameter in the range of 100-300 $\mu \mathrm{m}$, they are a little difficult to embed into the thicker walls of the gel. 


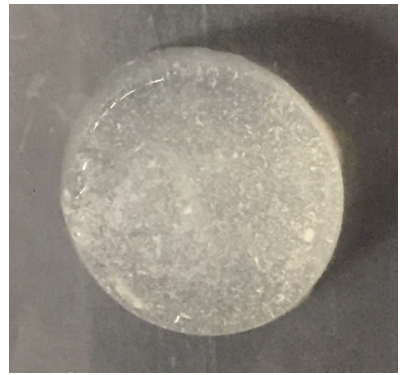

(a)

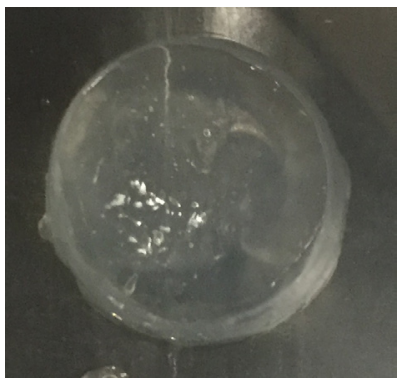

(c)

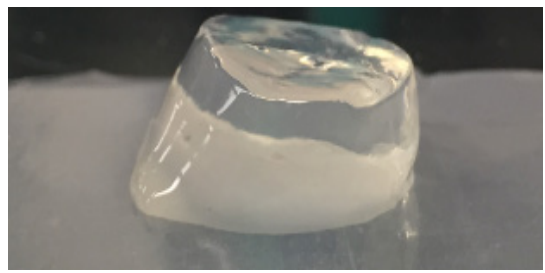

(e)

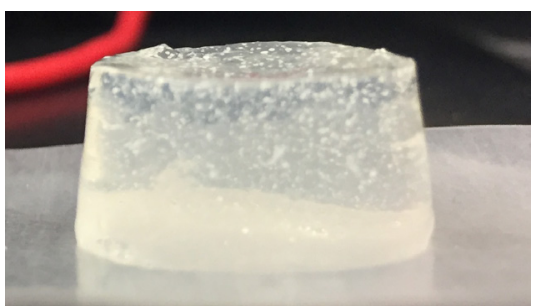

(b)

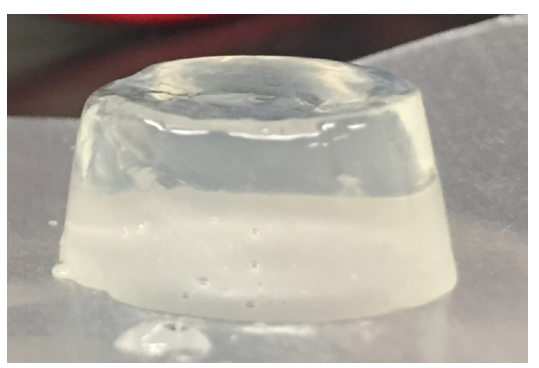

(d)

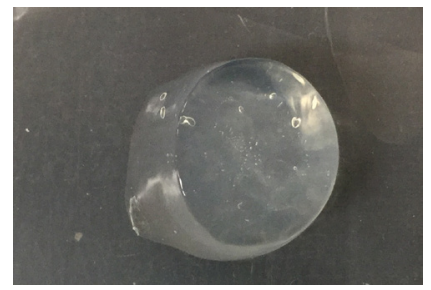

(f)

Figure 3.2 (a) and (b) shows hydrogel-microparticle composite. (c) and (d) shows blank hydrogel without microparticles. (e) and (f) depicts the ascorbic acid chitosan-agarose hydrogel.

\subsubsection{In vitro NO release profile study of microparticles hydrogel composite}

\subsubsection{Total NO release study via Light}

Total NO release from the hydrogel-microparticle $(500)$ and hydrogel-microparticle $(2500)$ is shown in fig. 5.2 (a) and (b) respectively. LED Light source (VAOL-5GSBY4, Mouser 
electric, Mansfield, TX) of $470 \mathrm{~nm}$ was used as a trigger to initiate NO production from hydrogel-microparticle composite. Light source had to be kept at an intensity suitable for maximum NO release within the detection limit of nitric oxide analyzer. That is why light source was kept at $4 \mathrm{~V}$ during the testing. Increasing the voltage exceeded the NO detection limit which is undesirable. The reason for this is to keeps the NO release in detection limit. NO release profile follows the same biphasic profile as NO release profile of the SNAP loaded microparticles.

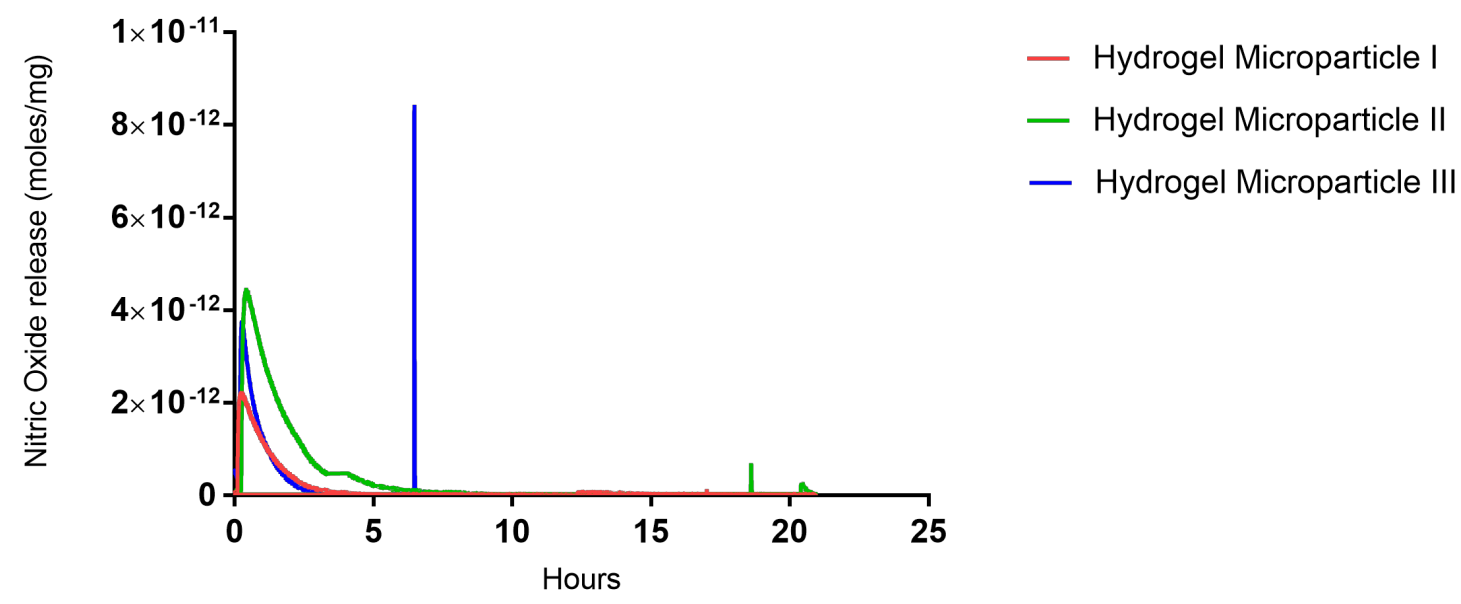

(a) Total NO release from microparticle (500 RPM)-hydrogel composite via Light 


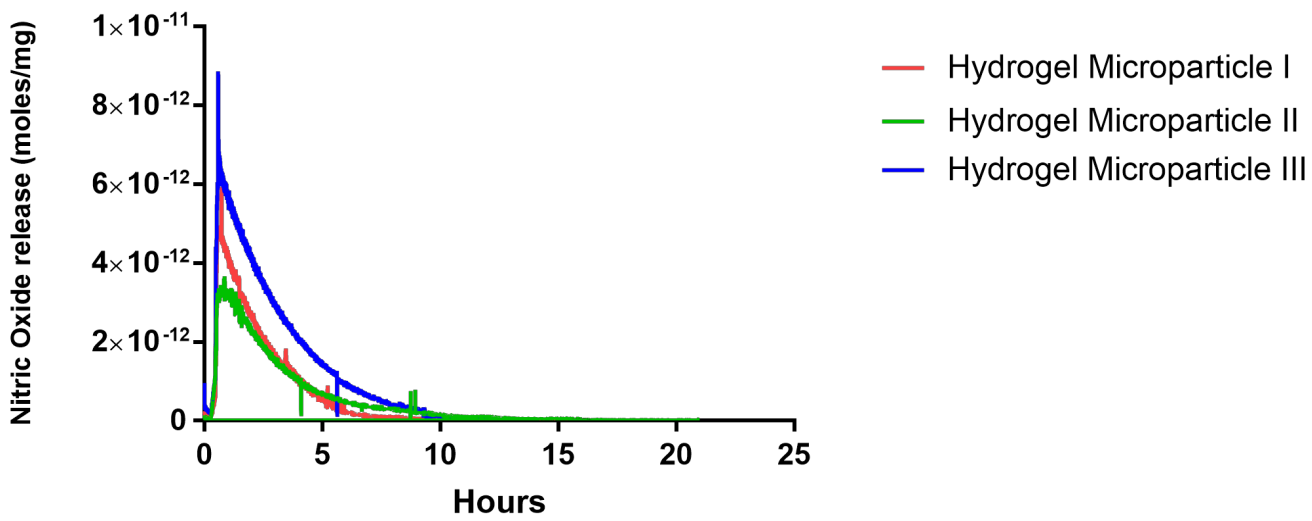

(b) Total NO release from microparticle (2500 RPM)-hydrogel composite via Light

Figure 3.3 Light initiated total NO release from (a) Hydrogel-microparticles(500) and (b) hydrogel-microparticles $(2500)$

The NO release rate of hydrogel-microparticle $(2500)$ composite is similar to the microparticle $(2500)$. The comparison of light initiated total NO release rate from Hydrogelmicroparticles $(500)$ and hydrogel-microparticles $(2500)$ is shown in Fig. 5.3. The initial average burst release is $46.3 \% \pm 6.1$ of total $\mathrm{NO}$ measured in hydrogel-microparticle $(2500)$ while release rate is faster in case of hydrogel-microparticle (500) composite with an initial burst release of $69.6 \% \pm 19.3$ of total NO measured. The burst release occurs for almost $2 \mathrm{~h}$. These results are perplexing as even more sustained NO release was expected. 


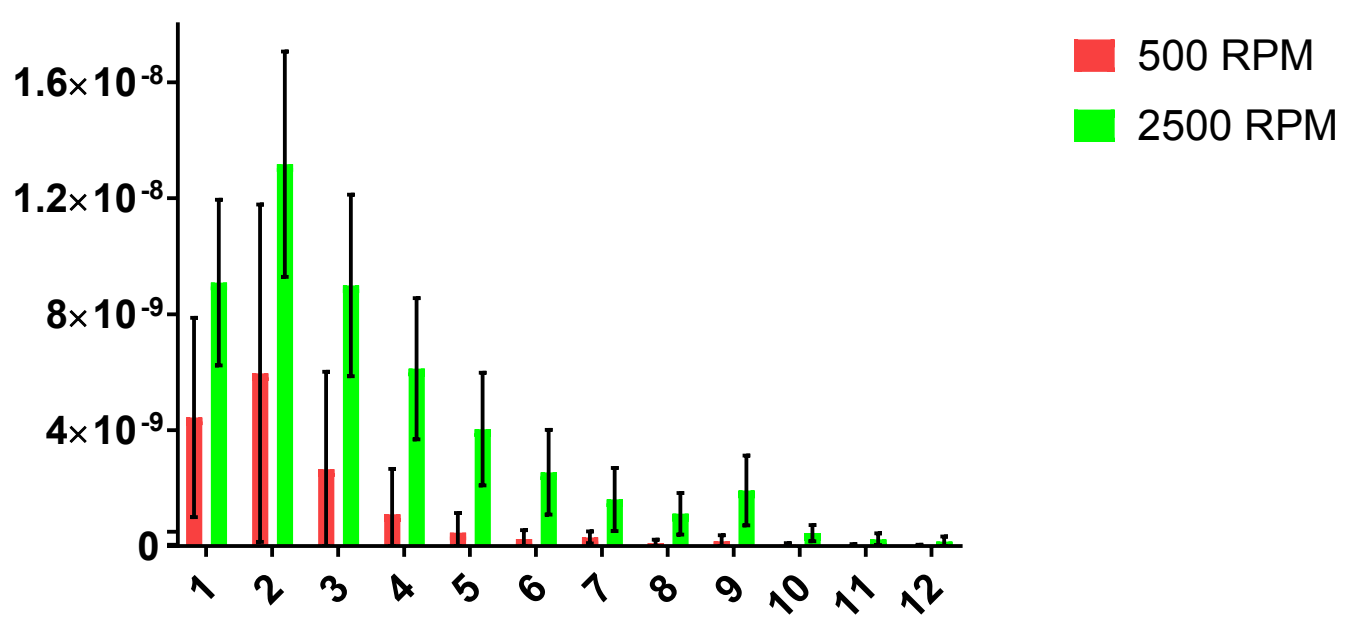

Hours

Comparison of Nitric oxide release rate between hydrogels (via light release)

Figure 3.4 comparison of light initiated total NO release rate from Hydrogelmicroparticles(500) and hydrogel-microparticles $(2500)$

\subsubsection{Effect of particle size on total NO release due to light in microparticle hydrogel composite}

The amount of NO released from the hydrogel-microparticle(500) and hydrogelmicroparticle $_{(2500)}$ were compared using two tail t-test. The results as shown in Fig. 5.4 displayed a significant difference in total NO release between both the composite systems $(\mathrm{p}=0.04)$. This can be attributed to the difference in size of microparticles which was also seen in only microparticle system.

Compared to only microparticles, less release was observed from both composites that may be either due to degradation of SNAP in the microparticle during hydrogel preparation or interaction between microparticle and hydrogel which reduces NO release. However, release from hydrogel-microparticle(500) composite was much lower than hydrogelmicroparticle $(2500)$ composite in relation their respective microparticle NO release. Using light as a trigger, the NO release from the hydrogel-microparticle $(500)$ was just $25.68 \% \pm$ 5.9 of total NO released from only microparticles(500). While, the NO released from the 
hydrogel-microparticle $(2500)$ was $50.3 \% \pm 14.5$ of total released NO from microparticle $(2500)$. This can be due to larger size of microparticle(500) which maybe degrade faster.

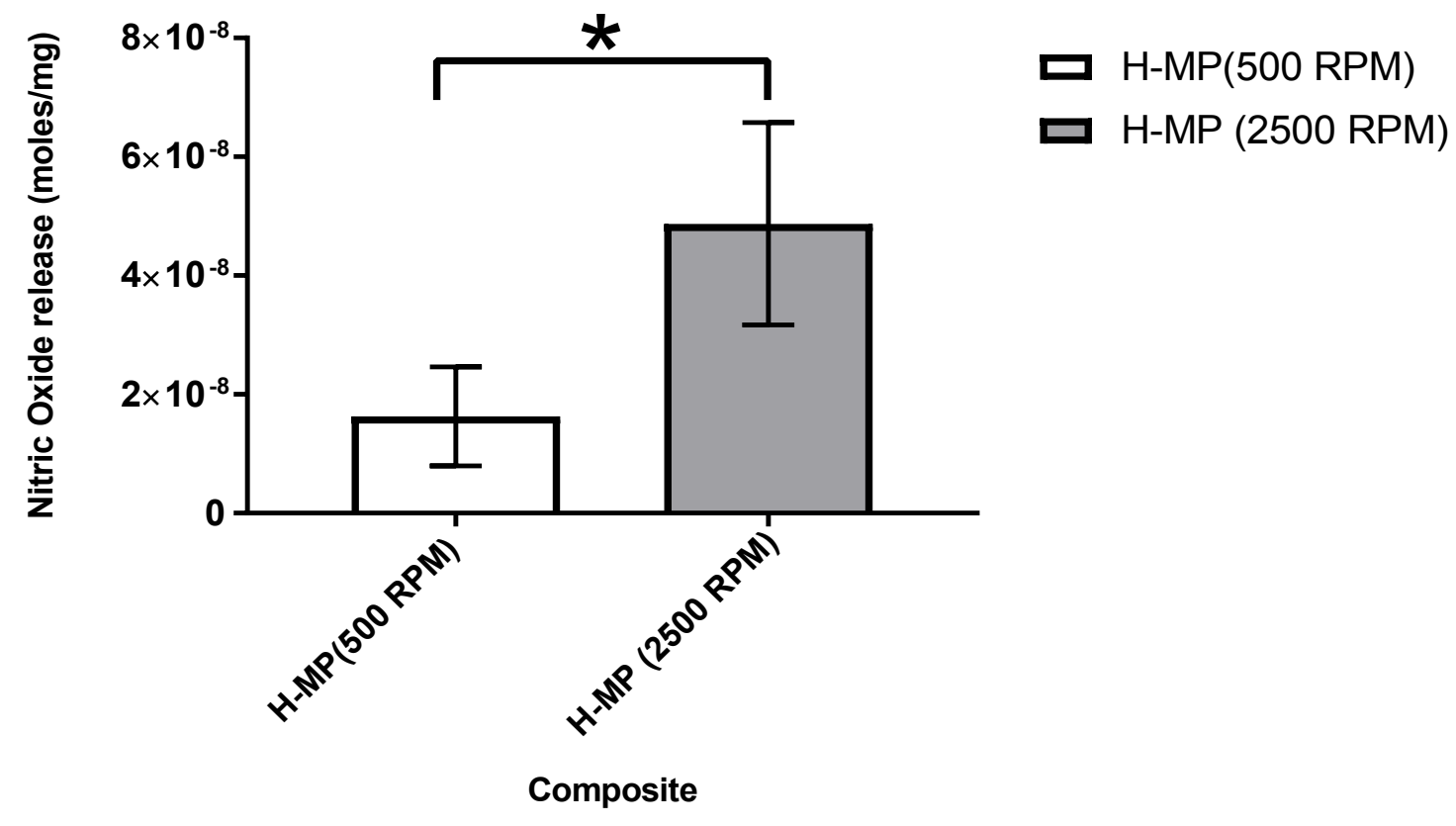

Total NO release from microparticle-hydrogel composite via Light

Figure 3.5 comparison of total NO release rate from the hydrogel-microparticle $(500)$ and hydrogel-microparticles $(2500)$

\subsubsection{Total NO release study via ascorbic acid hydrogel}

Another hydrogel system containing ascorbic acid prepared as described in method was used as an initiator for NO release study from the hydrogel-microparticle composite system. In this study, shown in fig. 5.5, ascorbic acid hydrogel was placed above the hydrogel-microparticle composite and total NO release was measured. A magnetic stirrer was used for proper mixing. Total NO release from the hydrogel-microparticle ${ }_{(500)}$ and hydrogel-microparticle $(2500)$ using ascorbic acid is shown in figure 5.6 (a) and (b) respectively. Same as microparticle study, only $100 \mathrm{mM}$ ascorbic acid was used in study. 
NO release profile follows the same biphasic profile as NO release profile of the SNAP loaded microparticles. However, in this study after the burst release, the slower release period was very short.

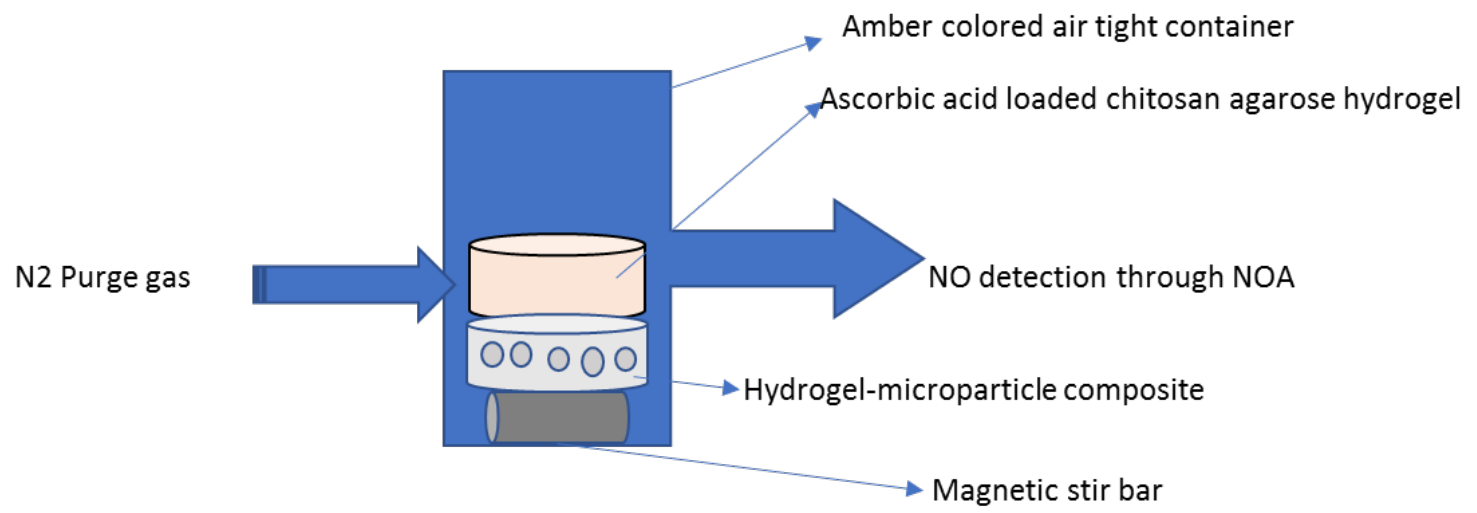

Figure 3.6 Illustration of ascorbic acid loaded chitosan agarose hydrogel system used for NO release study in hydrogel-microparticle composite

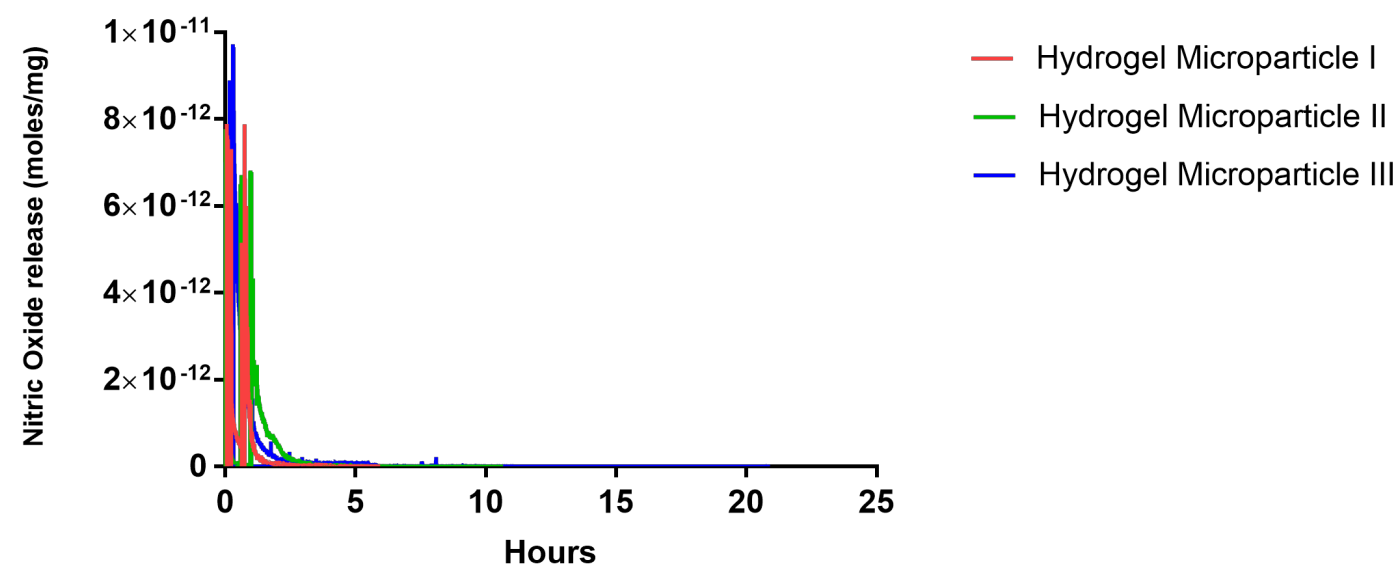

(a) Total NO release from microparticle (500 RPM)-hydrogel composite via Ascorbic acid hydrogel composite 


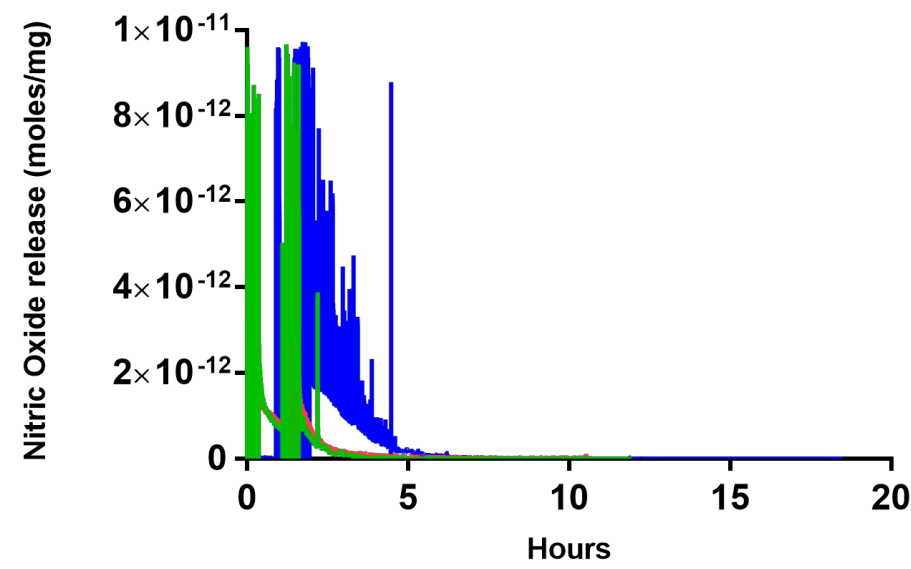

- Hydrogel Microparticle I

- Hydrogel Microparticle II

- Hydrogel Microparticle III

(b) Total NO release from microparticle(2500 RPM)-hydrogel composite via Ascorbic acid hydrogel composite

Figure 3.7 Ascorbic acid initiated total NO release rate from the (a) hydrogelmicroparticle(500) and (b) hydrogel-microparticles(2500)

The comparison of ascorbic acid initiated NO release rate from the hydrogelmicroparticle(500) and hydrogel microparticles(2500) is shown in Fig. 5.7. The hydrogelmicroparticle(2500) and hydrogel-microparticle(500) composite exhibit an average burst release of $38.6 \pm 12.3$ and $44.8 \% \pm 11.4$ respectively. The burst release time for hydrogelmicroparticle $(2500)$ is again $2 \mathrm{~h}$ but the total release time has decreased to almost $6 \mathrm{~h}$. In case of hydrogel-microparticle $(500)$ burst release is very short as well as the total release time. The nitric oxide release analysis through ascorbic acid hydrogel showed low total NO output. When compared to total NO output in microparticles, hydrogel-microparticle $(500)$ and hydrogel-microparticle $(2500)$ displayed only $30.2 \% \pm 12.4$ and $35.4 \% \pm 2.9$ NO output in relation to their respective microparticle counterparts. Even though more sustained NO release was expected in this case too, the low NO release may be due to any interaction of the hydrogel-microparticle composite with another ascorbic acid hydrogel. 
Comparison of Nitric oxide release rate between hydrogels (via ascorbic acid release)

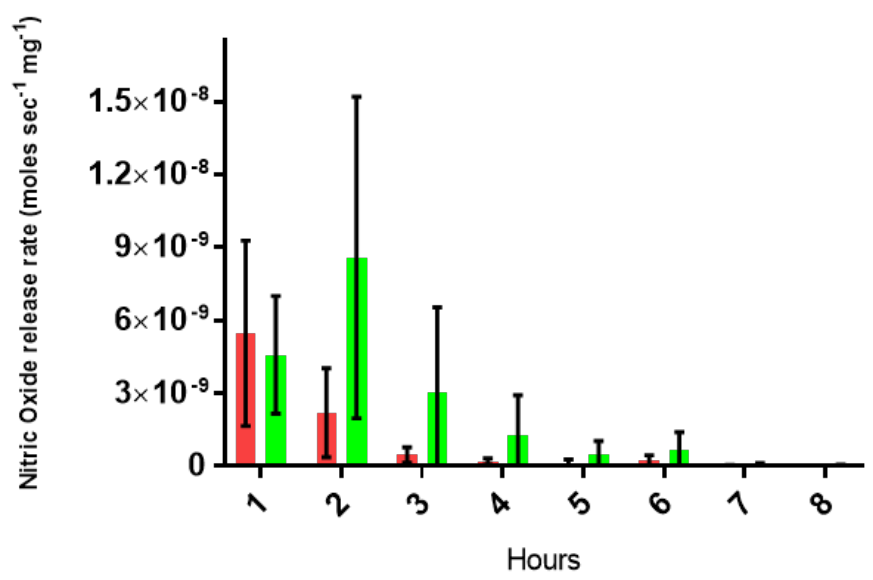

500 RPM

2500 RPM

Figure 3.8 comparison of ascorbic acid initiated NO release rate from the hydrogelmicroparticle $_{(500)}$ and hydrogel microparticles(2500)

\subsubsection{Effect of particle size on total NO release due to ascorbic acid hydrogel in microparticle hydrogel composite}

Similar to the previous study, the amount of NO released from the hydrogelmicroparticle $_{(500)}$ and hydrogel- $\operatorname{microparticle}_{(2500)}$ were compared using two tail t-test. The results as shown in in Fig. 5.8 displayed a significant difference in total NO output due to ascorbic acid hydrogel between both the composite systems $(p=0.01)$. Again, the significant difference in NO release between two different hydrogel-microparticle systems due to ascorbic acid can be attributed to the difference in size of microparticles which was also seen in total NO release in only microparticle system and light initiated NO release in hydrogel-microparticle composite system. Degradation of SNAP in the microparticle during hydrogel preparation or interaction between microparticle and hydrogel can be the reason for observed less release which reduces NO release. 


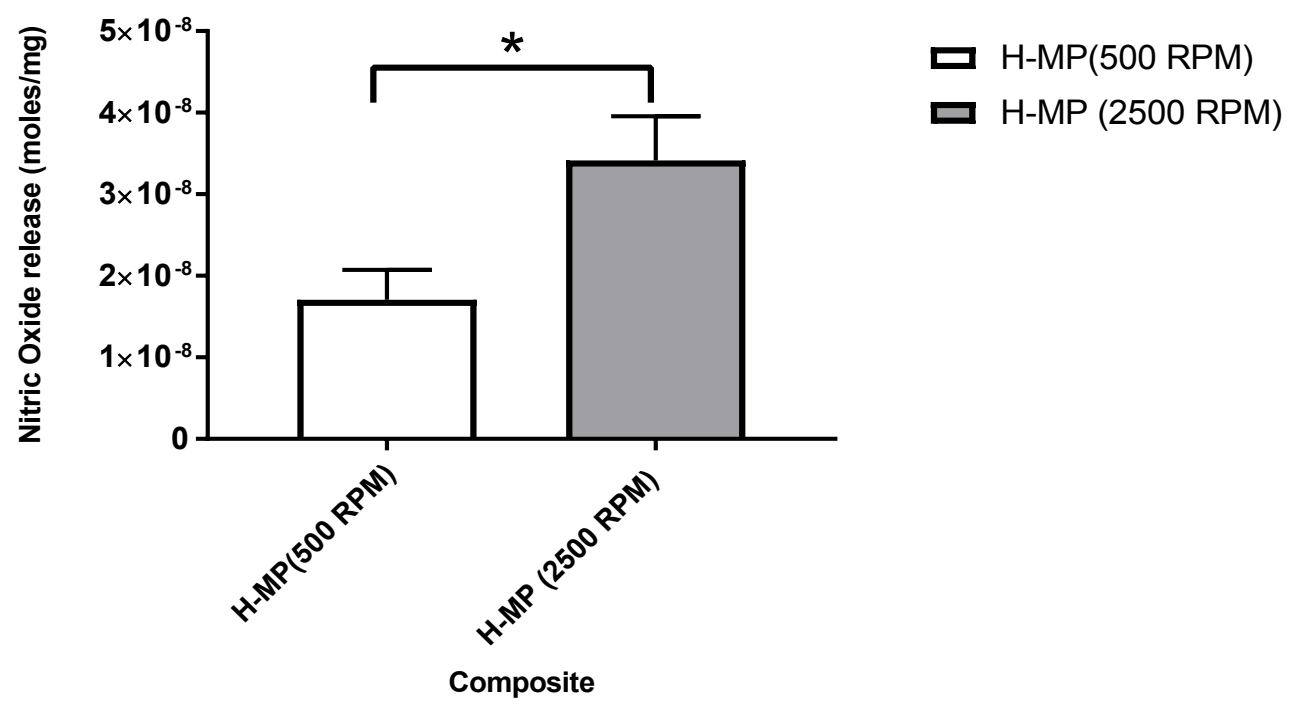

Total NO release from microparticle-hydrogel composite via Ascorbic acid hydrogel composite

Figure 3.9 comparison of total NO release from the hydrogel-microparticle(500) and hydrogel-microparticles $(2500)$

Table 4 shows the average total nitric oxide released from hydrogel-microparticle $(500)$ and hydrogel-microparticle $(2500)$ by exposure to light and ascorbic acid hydrogel. The average ratio of NO output of hydrogel-microparticle $(500)$ through light and ascorbic acid is $1.07 \pm$ 0.82 while average ratio of NO output of hydrogel-microparticle $(2500)$ through light and ascorbic acid is $1.41 \pm 0.29$. This shows that total NO release from both the hydrogelmicroparticle system by using both light and ascorbic acid hydrogel triggers are almost similar. 
Table 4 comparison of average total nitric oxide release from hydrogel-microparticle(500) and hydrogel-microparticle(2500) by exposure to light and ascorbic acid hydrogel

\begin{tabular}{|l|l|l|}
\hline \multirow{2}{*}{ Composition } & \multicolumn{2}{|c|}{ Average total NO release \pm s.d. } \\
\cline { 2 - 3 } & Exposure to light & $\begin{array}{l}\text { Exposure to ascorbic acid } \\
\text { hydrogel }\end{array}$ \\
\hline Hydrogel-microparticle $_{(500)}$ & $1.63 \pm 0.84 \times 10^{-8}$ & $1.71 \pm 0.37 \times 10^{-8}$ \\
\hline Hydrogel-microparticle $_{(2500)}$ & $4.87 \pm 1.70 \times 10^{-8}$ & $3.41 \pm 0.54 \times 10^{-8}$ \\
\hline
\end{tabular}

\subsection{Discussion}

\subsubsection{Hydrogel-microparticle composite}

In this study, a SNAP hydrogel-microparticle composite was designed in order to modify drug release profile of the microparticles. Used hydrogel was prepared from chitosan and agarose in acetic acid solution. During hydrogel preparation step microparticles were introduced in a form of microparticle suspension. Control gel and composite gel both had regular cylindrical shape. Small microparticles with less diameter are easily embedded in to the microparticles, however bigger microparticles with large diameter were visible in the hydrogel even after making them in to a suspension. Most of the microparticles are in the range of 100-300 $\mu \mathrm{m}$ which can be the main reason for visible microparticles in hydrogel.

Drug release test from the hydrogel-microparticle composite was done using two different triggers for nitric oxide release. Light and ascorbic acid hydrogel were used to initiate NO production from the SNAP loaded inside the microparticles which are embedded in the hydrogel.

Light initiated NO release from the composite showed fast release in hydrogels embedded with microparticle $(500)$ whereas the NO release from the hydrogels embedded with microparticles (2500) was almost similar to the microparticles(2500). Ascorbic acid hydrogel system was also used in the study of NO release from the composite hydrogel. Ascorbic acid initiated NO release from both the hydrogel microparticle composite exhibited very 
fast NO release. Like NO release study done with the light, these results were not expected since more sustained NO release was expected. Although NO release is hard to predict due to possible interaction between the components, hydrogel forms an extra barrier between the NO release triggers and SNAP. Therefore, a reasonable expectation is slower release profile in both the cases. However, hydrogels do not always necessarily delay the release from the microparticles as seen in case of poly (lactide) (PLA) microparticles embedded in poly (ethylene glycol) - poly (H-caprolactone) - poly (ethylene glycol) hydrogel that exhibited slightly accelerated release (23). There can be some reasons for unexpected behavior. First can be microparticle size. The explanation of this can be bigger size of the microparticles. The size of microparticle $(500)$ is pretty large which can prevent them from fully encapsulating inside the mesh of the formed hydrogel. Every hydrogel has a certain mesh size. If the size of the microparticles is bigger than mesh, they will be difficult to embed and remain close to the hydrogel boundaries resulting in easy and fast release of the drug. Another reason can be testing parameters. In the total NO release analysis of microparticles all triggers were used, but case of in hydrogel only light and ascorbic acid were used separately.

Apart from the rate of release, hydrogel-microparticle composite showed overall less amount of NO release compared to their microparticle counterparts. Usually, it is taken in to account that adding any more processing parameter can result in decrease of degradation of the matrix or the drug. In this case, the drug i.e. SNAP is sensitive to light, any trace of metal ions $\left(\mathrm{Cu}^{+}\right)$and ascorbic acid. Hydrogel-microparticle composite manufacturing process could cause SNAP loss from the microparticles. There is a high possibility that loss of SNAP occurs during the preparation of the homogenous microparticle suspension. The degradation of SNAP in the microparticles could have occurred due to their presence in the suspension or the thermal decomposition of SNAP microparticles when the microparticle chitosan agarose suspension was kept in water bath at $60^{\circ} \mathrm{C}$. The other reason can be polymer-polymer interactions. In general, it is hard to predict drug release from the microparticles, then it is obvious that it is more complicated in case of composite gels that has different components involved. Composite gel used in this study involves three 
different polymers PLLA, chitosan and agarose. Interaction between any of these components with each other may accelerate the degradation of the matrix and NO release.

\subsection{Summary}

In this study, it has been shown that NO donor SNAP encapsulated PLLA microparticles can be embedded into thermosensitive chitosan-agarose hydrogel. These hydrogelmicroparticle composite have demonstrated to release nitric oxide on exposure to light and ascorbic acid. The drug release rate from these composites was fast compared to the microparticles. Based on the size of microparticles incorporated in hydrogel, the hydrogelmicroparticles(2500) composites exhibited significant difference in NO release from hydrogel-microparticles(500). These composites can be successfully used as wound dressing utilizing the properties of chitosan and agarose in accelerating wound healing as well as antibacterial activity of nitric oxide.

\subsection{References}

1. Atiyeh BS, Gunn SW, Hayek SN. State of the art in burn treatment. World journal of surgery. 2005;29(2):131-48.

2. Metcalfe AD, Ferguson MW. Bioengineering skin using mechanisms of regeneration and repair. Biomaterials. 2007;28(34):5100-13.

3. Nicodemus GD, Bryant SJ. Cell encapsulation in biodegradable hydrogels for tissue engineering applications. Tissue Engineering Part B: Reviews. 2008;14(2):149-65.

4. $\quad \mathrm{Yu}$ L, Ding J. Injectable hydrogels as unique biomedical materials. Chemical Society Reviews. 2008;37(8):1473-81.

5. Balakrishnan B, Mohanty M, Umashankar P, Jayakrishnan A. Evaluation of an in situ forming hydrogel wound dressing based on oxidized alginate and gelatin. Biomaterials. 2005;26(32):6335-42. 
6. Boateng JS, Matthews KH, Stevens HN, Eccleston GM. Wound healing dressings and drug delivery systems: a review. Journal of pharmaceutical sciences. 2008;97(8):2892-923.

7. Carpenter AW, Schoenfisch MH. Nitric oxide release: Part II. Therapeutic applications. Chemical Society Reviews. 2012;41(10):3742-52.

8. Lundberg JO, Gladwin MT, Weitzberg E. Strategies to increase nitric oxide signalling in cardiovascular disease. Nature reviews Drug discovery. 2015;14(9):623-41.

9. Mocellin S, Bronte V, Nitti D. Nitric oxide, a double edged sword in cancer biology: searching for therapeutic opportunities. Medicinal research reviews. 2007;27(3):317-52.

10. Chandrawati R. Enzyme-responsive polymer hydrogels for therapeutic delivery. Experimental Biology and Medicine. 2016;241(9):972-9.

11. Zhao Q, Zhang J, Song L, Ji Q, Yao Y, Cui Y, et al. Polysaccharide-based biomaterials with on-demand nitric oxide releasing property regulated by enzyme catalysis. Biomaterials. 2013;34(33):8450-8.

12. Bohl KS, West JL. Nitric oxide-generating polymers reduce platelet adhesion and smooth muscle cell proliferation. Biomaterials. 2000;21(22):2273-8.

13. Shishido SIM, Seabra AB, Loh W, de Oliveira MG. Thermal and photochemical nitric oxide release from S-nitrosothiols incorporated in Pluronic F127 gel: potential uses for local and controlled nitric oxide release. Biomaterials. 2003;24(20):3543-53.

14. Seabra A, Fitzpatrick A, Paul J, De Oliveira M, Weller R. Topically applied Snitrosothiol-containing hydrogels as experimental and pharmacological nitric oxide donors in human skin. British Journal of Dermatology. 2004;151(5):977-83.

15. Vercelino R, Cunha TM, Ferreira ES, Cunha FQ, Ferreira SH, de Oliveira MG. Skin vasodilation and analgesic effect of a topical nitric oxide-releasing hydrogel. Journal of Materials Science: Materials in Medicine. 2013;24(9):2157-69.

16. Schanuel FS, Santos KSR, Monte-Alto-Costa A, de Oliveira MG. Combined nitric oxide-releasing poly (vinyl alcohol) film/F127 hydrogel for accelerating wound healing. Colloids and Surfaces B: Biointerfaces. 2015;130:182-91.

17. Park J, Kim J, Singha K, Han D-K, Park H, Kim WJ. Nitric oxide integrated polyethylenimine-based tri-block copolymer for efficient antibacterial activity. Biomaterials. 2013;34(34):8766-75. 
18. Kang Y, Kim J, Lee YM, Im S, Park H, Kim WJ. Nitric oxide-releasing polymer incorporated ointment for cutaneous wound healing. Journal of Controlled Release. 2015;220:624-30.

19. Kim J, Lee Y, Singha K, Kim HW, Shin JH, Jo S, et al. NONOatespolyethylenimine hydrogel for controlled nitric oxide release and cell proliferation modulation. Bioconjugate chemistry. 2011;22(6):1031-8.

20. Cao Z, Gilbert RJ, He W. Simple Agarose- Chitosan gel composite system for enhanced neuronal growth in three dimensions. Biomacromolecules. 2009;10(10):2954-9.

21. Miguel SP, Ribeiro MP, Brancal H, Coutinho P, Correia IJ. Thermoresponsive chitosan-agarose hydrogel for skin regeneration. Carbohydrate polymers. 2014;111:366-73.

22. Dillon GP, Yu X, Sridharan A, Ranieri JP, Bellamkonda RV. The influence of physical structure and charge on neurite extension in a 3D hydrogel scaffold. Journal of Biomaterials Science, Polymer Edition. 1998;9(10):1049-69.

23. Fan M, Guo Q, Luo J, Luo F, Xie P, Tang X, et al. Preparation and in vitro characterization of dexamethasone-loaded poly (d, l-lactic acid) microspheres embedded in poly (ethylene glycol)-poly ( $\varepsilon$-caprolactone)-poly (ethylene glycol) hydrogel for orthopedic tissue engineering. Journal of biomaterials applications. $2013 ; 28(2): 288-97$. 


\section{Conclusion and future propositions}

Chronic wounds are a major burden to the healthcare industry. Current treatments available are not satisfactory to treat chronic wounds due to their inability to prevent bacterial infection and stimulate growth of healthy cells around wounded tissue. There is an urgency to develop new methods of infection control as some bacterial strains (also known as superbugs) continue to develop resistance against antibiotics. Therefore, there has been an interest in exploring the role of a small molecule nitric oxide naturally produced by cells during wound healing and development of NO delivery devices.

The main aim of this work was to develop stable polymeric systems capable of delivering nitric oxide in a sustained and controllable manner for their potential application as wound healing materials. There were two goals of this study: (a) synthesize and study an efficient microparticle release system encapsulating a NO donor SNAP and, (b) to develop hydrogel-microparticle composite material to modify the NO release profile. In this study, SNAP was successfully encapsulated in the PLLA microparticles using solvent evaporation water-in-oil-water double emulsion at a rotor stirring speed of $500 \mathrm{rpm}$ and $2500 \mathrm{rpm}$. In this microparticle formulation, $\mathrm{HCl}$ was added to the PVA aqueous phase of the emulsion to prevent SNAP degradation as it is more stable at acidic $\mathrm{pH}$. The microparticles produced at $500 \mathrm{rpm}$ and $2500 \mathrm{rpm}$ had particle sizes of $256 \pm 11.7 \mu \mathrm{m}$ and $207 \pm 19.7 \mu \mathrm{m}$ with an encapsulation efficiency of $35.2 \pm 5.6 \%$ and $33.0 \pm 1.4 \%$ respectively. There is a significant difference in release profile between both microparticles due to difference in microparticle size. The microparticles prepared at 500 and $2500 \mathrm{rpm}$ released an average of $6.11 \pm 1.71 \times 10^{-8}$ and $8.43 \pm 2.92 \times 10^{-8}$ of total encapsulated nitric oxide for almost $9 \mathrm{~h}$ and $13 \mathrm{~h}$ respectively on exposure to light, copper and ascorbate. The microparticles also demonstrated controlled NO release on exposure of different intensities of light which can be used to regulate the amount of nitric oxide according to the requirement. The microparticles had an accelerated NO release and higher burst rate which may be due to irregularities on surface and collapsed microparticles because of air drying. 
Also, SNAP loaded hydrogel-microparticle composites were developed. The hydrogelmicroparticle composite also showed sensitivity to light and ascorbic acid and allow SNAP decomposition and NO release on exposure to them. The hydrogel-microparticle $(500)$ and hydrogel- microparticle $(2500)$ composite released an average total of $1.63 \pm 0.84 \times 10^{-8}$ and $4.87 \pm 1.70 \times 10^{-8}$ nitric oxide respectively with only light as a trigger. On the other hand, ascorbic acid chitosan-agarose hydrogel used for release study demonstrated an average total of $1.71 \pm 0.37 \times 10^{-8}$ and $3.41 \pm 0.54 \times 10^{-8}$ nitric oxide release from the hydrogelmicroparticle $_{(500)}$ and hydrogel-microparticle $(2500)$ composite. Nitric oxide release from the composite gel was faster compared to the only microparticles which is likely due to larger size of microparticles or problems with the preparation of the microparticle suspension. A significant difference in release profile between both hydrogel microparticle composite systems was observed. The NO release from the composite for several hours may be useful for short term applications where fast release is needed such as wound healing.

Based on these results, future work can be done to further determine if the SNAP loaded microparticles and hydrogel-microparticle composites are effective in delivering enough nitric oxide for inhibiting the bacterial growth. A bacterial study can provide major evidence regarding the antibacterial activity of these microparticles and composites as in the wound healing process antibacterial activity of NO is the most critical feature. Additionally, it can be studied if this type of dosage (high initial NO release for shorter time period) is effective on bacterial inhibition. Furthermore, in vitro studies such as in endothelial, epithelial cells and in vivo studies in animals can be done after the successfully determining the necessary dosage and surface flux necessary to impede bacterial infection in wound healing process.

For future improvements in the development of this system, it is suggested to make various changes to the formulation of this microparticles system to sustain nitric oxide release for longer period. Some other polymers such as poly (lactic acid-co-glycolic acid) (PLGA) can be used for encapsulation of nitric oxide donor using the encapsulation technique described in this thesis. Alternatively, microparticles could be coated with the drug free polymer (same or another) to slow down the release. Microparticles can be produced at different 
stirring rates to further determine its effect in SNAP encapsulation. It would be worth to try encapsulating more amount of SNAP during production of microparticles to study efficient loading of microparticles. Another type of nitric oxide donors such as SNAPcyclam developed by our lab can be used in these microparticle system as it degrades slowly compared to SNAP (1). Copper is main metal ion responsible for the degradation of SNAP and NO release which is studied in his research, however, activity of other metal ions such as Fe, Zn etc. on SNAP microparticles and composites can also be studied for NO release from these microparticles (2).

Additionally, further testing of the hydrogel-microparticle composite is advisable. There are various published articles which have studied sustained modified drug release profiles of microparticles embedded in the hydrogels (3). In this thesis, only preliminary nitric oxide release test was performed from both microparticles and hydrogel-microparticle composite. Further testing such as mechanical and stability of the composite can be performed.

\subsection{References}

1. McCarthy CW, Goldman J, Frost MC. Synthesis and Characterization of the Novel Nitric Oxide (NO) Donating Compound, S-nitroso-N-acetyl-D-penicillamine Derivatized Cyclam (SNAP-Cyclam). ACS applied materials \& interfaces. 2016;8(9):5898-905.

2. McCarthy CW, Guillory RJ, Goldman J, Frost MC. Transition-Metal-Mediated Release of Nitric Oxide (No) from S-Nitroso-N-Acetyl-D-Penicillamine (SNAP): Potential Applications for Endogenous Release of No at the Surface of Stents Via Corrosion Products. ACS applied materials \& interfaces. 2016;8(16):10128-35.

3. Hoare TR, Kohane DS. Hydrogels in drug delivery: progress and challenges. Polymer. 2008;49(8):1993-2007. 


\section{Appendix A}

\section{Letters of Permission}

This part shows that I obtained permission from the publishers and have the right to use the related materials.

This letter shows that I have the right to use the related materials for Fig. 1.1

$1 / 25 / 2018$

Michigan Technological University Mail - Request \#9131-7998524 Resolved (Copyright permission for graduate thesis)

Michigan Tech

Nikhil Mittal <nmittal@mtu.edu>

\section{Request \#9131-7998524 Resolved (Copyright permission for graduate thesis)}

support@services.acs.org <support@services.acs.org>

Wed, Jan 24, 2018 at 2:53 PM

Reply-To: support@services.acs.org

To: "nmittal@mtu.edu" <nmittal@mtu.edu>

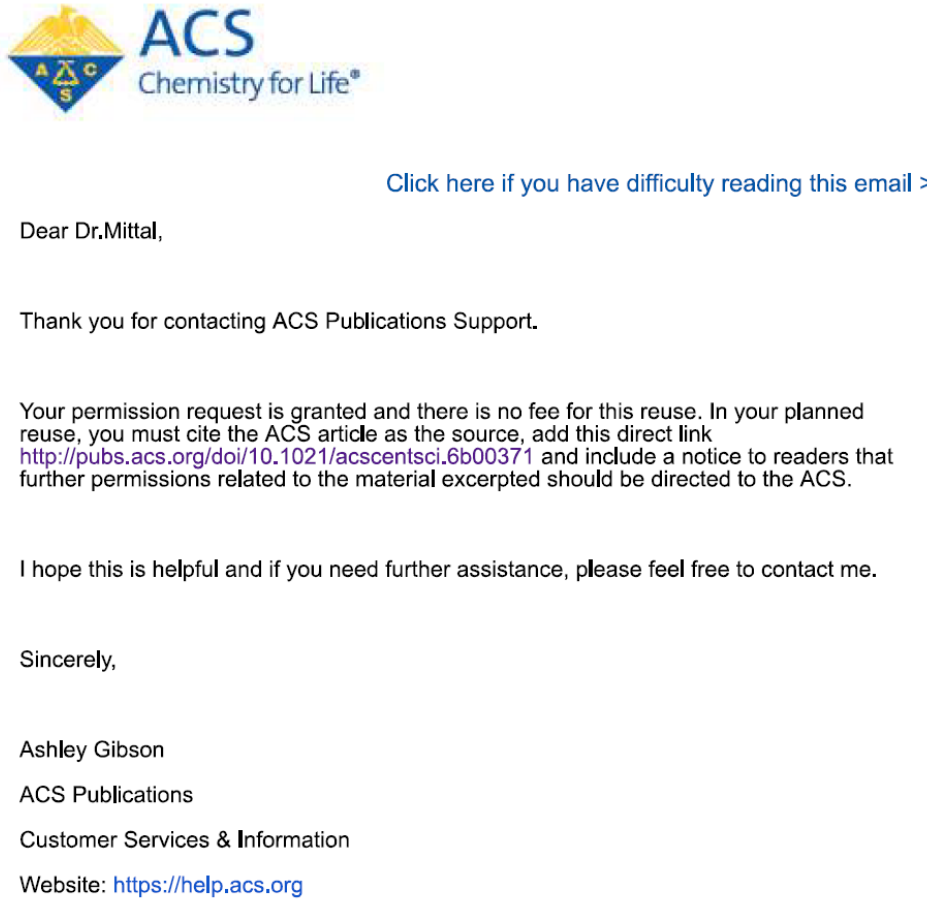

Your help request has been resolved. If you have further issues regarding this matter, please let us know by responding to this email. Please note that this request will autoclose in 14 days. If you need to contact us after 14 days regarding this matter please submit a new help request and refer to this help request number. 


\section{E-mail Information:}

Attachments

$\mathrm{CC}$

\section{Request Information:}

Request \# 9131-7998524

Date Created 1/24/2018 01:47 PM EST

Summany Copyright permission for graduate thesis

Hello,

I am a graduate student at Michigan Technological University in biomedical engineering department. I am working on my thesis titled An injectable thermosensitive biodegradable hydrogel embedded with SNAP containing PLLA microparticles for sustained nitric oxide (NO) delivery for wound healing.

I am writing to ask permission to use a figure from the research article "Nanotechnology-Driven Therapeutic Interventions in Wound Healing: Potential Uses and Applications" published in ACS central science in my thesis. The figure I want to use is Figure 1. Phases of cutaneous wound healing depicting the cells and molecules responsible Details for the regaining of a healthy barrier.

Here is the link to this research article: http://pubs.acs.org/doi/10.1021/acscentsci.6b00371

I will be really grateful if I can get permission to use this figure in my graduate thesis.

Thanking you

Sincere Regards,

Nikhil Mittal

Graduate student

Biomedical Engineering

Michigan Technological University[https://my-email-

signature.link/signature.gif $\mathrm{u}=220014 \& \mathrm{e}=16704394 \& \mathrm{v}=$

87179b81059d0768f262db11b719dc

4ea45994e9456dac8257833c67d62eb7d6]

To update or check the status of this request:

Click here to access this request online. 
This letter shows that I have the right to use the related materials for Fig. 1.2

$1 / 24 / 2018$

RightsLink Printable License

ELSEVIER LICENSE

TERMS AND CONDITIONS

Jan 24,2018

This Agreement between Michigan Technological Univ -- Nikhil Mittal ("You") and

Elsevier ("Elsevier") consists of your license details and the terms and conditions provided by Elsevier and Copyright Clearance Center.

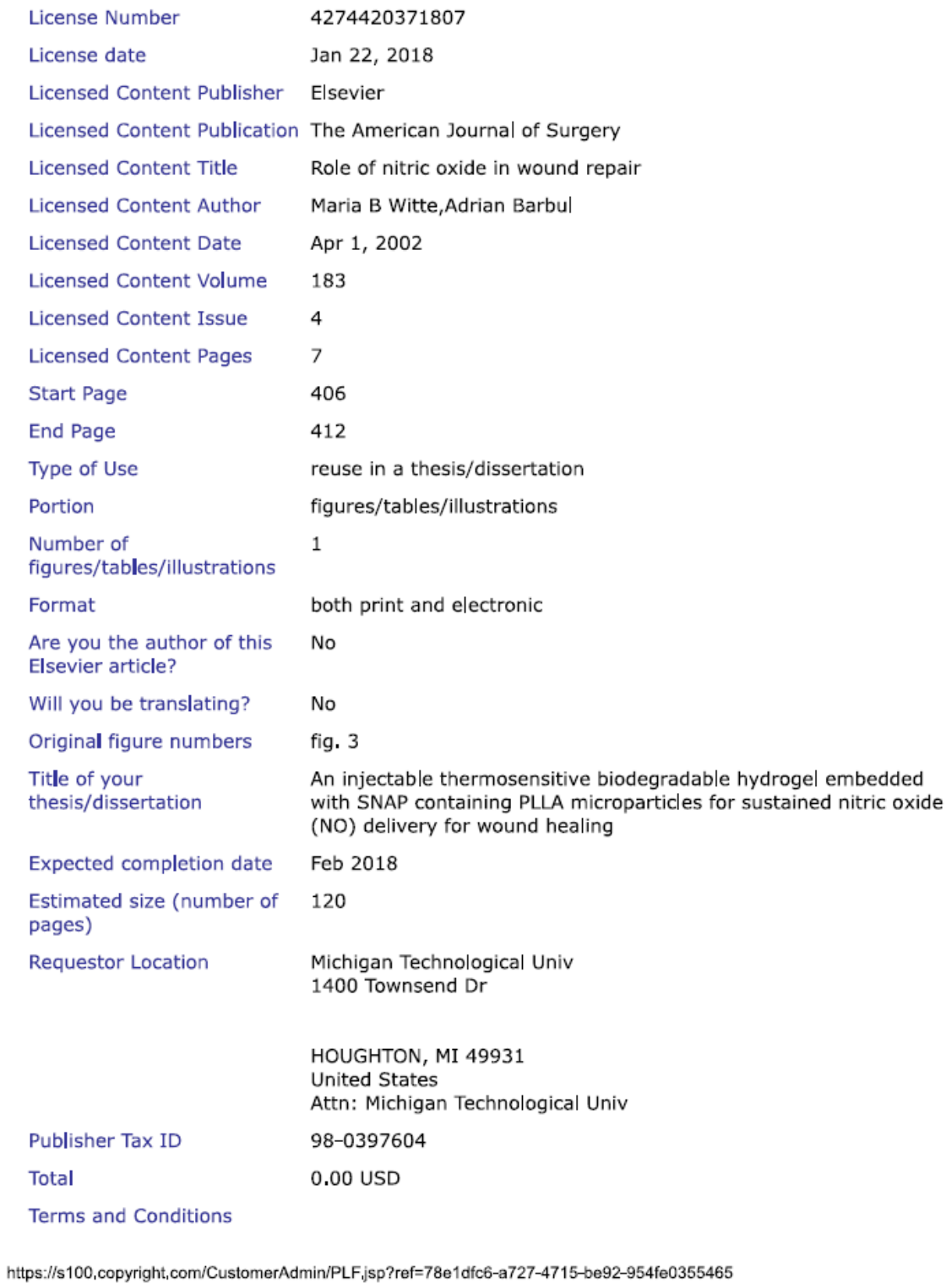

